\title{
ENRICO VITALI
}

\section{Convergence of unilateral convex sets in higher order Sobolev spaces}

\author{
Annales de la faculté des sciences de Toulouse $5^{e}$ série, tome 11, \\ no 3 (1990), p. 93-149 \\ <http://www.numdam.org/item?id=AFST_1990_5_11_3_93_0>
}

(C) Université Paul Sabatier, 1990, tous droits réservés.

L'accès aux archives de la revue «Annales de la faculté des sciences de Toulouse » (http://picard.ups-tlse.fr/ annales/) implique l'accord avec les conditions générales d'utilisation (http://www.numdam.org/conditions). Toute utilisation commerciale ou impression systématique est constitutive d'une infraction pénale. Toute copie ou impression de ce fichier doit contenir la présente mention de copyright.

\section{NumDam}

Article numérisé dans le cadre du programme Numérisation de documents anciens mathématiques http://www.numdam.org/ 


\title{
Convergence of unilateral convex sets in higher order Sobolev spaces
}

\author{
ENRICO Vitali ${ }^{(1)}$
}

RÉSUMÉ. - Nous obtenons une caractérisation de la convergence au sens de Mosco dans la classe des convexes unilatéraux de $W_{0}^{m, p}(\Omega)$ $(m \geq 1)$ de la forme $\left\{u \in W_{0}^{m, p}(\Omega) \mid u \geq \psi\right.$ sur $\left.\Omega\right\}$. Étant donnés les obstacles $\left(\psi_{h}\right)_{h}$ et $\psi$, cette caractérisation s'exprime par la convergence au sens de Mosco des ensembles unilatéraux associés aux fonctions caractéristiques des ensembles de niveau $\left\{\psi_{h}>t\right\}$ et $\{\psi>t\}$. Dans le cas $p=2$, en développant ce résultat nous obtenons des conditions nécessaires et suffisantes formulées au moyen de la convergence des $(m, 2)$-capacités des intersections $\left\{\psi_{h}>t\right\} \cap \boldsymbol{A}$ entre les ensembles de niveau et une convenable famille d'ouverts $A$.

Abstract. - We establish a characterization of the Mosco convergence for the class of unilateral convex sets in $W_{0}^{m, p}(\Omega)(m \geq 1)$ of the form $\left\{u \in W_{0}^{m, p}(\Omega) \mid u \geq \psi\right.$ on $\left.\Omega\right\}$. For given obstacles $\left(\psi_{h}\right)_{h}$ and $\psi$ this characterization is expressed in terms of the Mosco convergence of the unilateral sets determined by the characteristic functions of the level sets $\left\{\psi_{h}>t\right\}$ and $\{\psi>t\}$. In the case $p=2$ we further develop this result obtaining necessary and sufficient conditions in terms of the convergence of the $(m, 2)$-capacities of the intersections $\left\{\psi_{h}>t\right\} \cap A$ of the level sets with a suitable family of open sets $A$.

\section{Introduction}

The purpose of this paper is to give necessary and sufficient conditions for the convergence in the sense of Mosco of a sequence of convex sets of (unilateral) obstacle type in higher order Sobolev spaces.

(1) Scuola Internazionale Superiore di Studi Avanzati (S.I.S.S.A.), via Beirut 4-34014 Trieste (Italy);

permanent address : Università degli Studi di Parma, Dipartimento di Matematica, via M. D'Azeglio 85 - 43100 Parma (Italy). 
This notion of convergence was introduced by U. Mosco in [22] for the class of closed convex subsets of a reflexive Banach space and, through the epigraphs, for the class of convex lower semicontinuous functionals. The range of application covers a number of situations in perturbation or approximation of optimization problems (see [22], [23] and [3]). In particular, if $A$ is a (possibly non-linear) operator from a reflexive Banach space $X$ into its dual and $\left(K_{h}\right)$ is a sequence of convex subsets of $X$ which converges to a set $K$ in the sense of Mosco, then, under some natural assumptions on $A$, it can be proved that the solutions $u_{h}$ of the variational inequalities

$$
u_{h} \in K_{h}, \quad\left\langle A\left(u_{h}\right), v-u_{h}\right\rangle \geq 0 \quad \text { for every } v \in K_{h},
$$

converge strongly in $X$ to the solution $u$ of the variational inequality

$$
u \in K, \quad\langle A(u), v-u\rangle \geq 0 \quad \text { for every } v \in K .
$$

Therefore, a study was developed to determine significant conditions which could imply the Mosco convergence (see, for instance, [25]). Here we focus our attention on the important class of the convex subsets of $W_{0}^{m, p}(\Omega)\left(\Omega\right.$ open subset of $\left.\mathbb{R}^{n}, m \geq 1,1<p<+\infty\right)$ associated to an obstacle function, i.e. of the form $K_{0}(\psi)$, where $\psi: \Omega \rightarrow[-\infty,+\infty]$ is any function, and

$$
K_{0}(\psi)=\left\{u \in W_{0}^{m, p}(\Omega) \mid u \geq \psi \text { on } \Omega\right\} .
$$

We point out that in order to consider also thin obstacles $\psi$, namely obstacles given on sets of Lebesgue measure zero, we have to pass to the more refined concept of $C_{m, p^{-}}$-capacity (defined from the norm of $W^{m, p}\left(\mathbb{R}^{n}\right)$ ) and specify, up to sets of null capacity, both the values taken by $u$ and the set where the inequality $u \geq \psi$ has to be considered.

Given a sequence $\left(\psi_{h}\right)$ of obstacles, it is clear that a strong enough convergence of $\left(\psi_{h}\right)$ to $\psi$ implies the convergence of $\left(K_{0}\left(\psi_{h}\right)\right)$ to $K_{0}(\psi)$. The question is then to find minimal assumptions on the obstacles which guarantee the Mosco convergence.

In this connection, for the case $m=1$, we must mention the result obtained by H. Attouch and C. Picard in [5], which unifies previous studies on the same line (see the references in [9]). As a consequence, it is proved that the convergence of the obstacles in a suitable $L^{p}$-space constructed by 
means of the $C_{1, p}$-capacity, as well as the weak convergence of $\left(\psi_{h}\right)$ to $\psi$ in $W_{0}^{1, s}(\Omega)$ for some $s>p$, turn out to be sufficient to yield the Mosco convergence of the corresponding convex sets (see also [6]).

These results (quite satisfactory for $C_{1, p}$-quasi continuous obstacles) were refined, by means of new techniques, by G. Dal Maso in [9], where necessary and sufficient conditions are established for the convergence in the sense of Mosco of a sequence $\left(K_{0}\left(\psi_{h}\right)\right)$ in $W_{0}^{1, p}(\Omega)$. Here no regularity on the obstacles is assumed.

In this paper we aim to find an extension of the characterization given in [9] to the case of higher order Sobolev spaces. More precisely, we begin by presenting, in section 2, some properties of the class of subsets of $W_{0}^{m, p}(\Omega)$ we deal with. Here, on the basis of the concept of unilateral convex set in $W_{0}^{1, p}(\Omega)$ (see [4]), we introduce an analogous notion for $W_{0}^{m, p}(\Omega)$ (requiring that, if $u$ and $v$ are in the set, then the same holds for every $w \geq u \wedge v$ (minimum between $u$ and $v$ )). It is proved that every closed unilateral convex set in $W_{0}^{m, p}(\Omega)$ is a convex set of obstacle type. This extends the corresponding result given in [4] for the case $m=1$, and allows us to obtain the closedness, under the Mosco convergence, of the class of obstacles constraints in $W_{0}^{m, p}(\Omega)$.

The subsequent sections can be divided into two parts. In the first one (sections 3 and 4 ) we take into consideration the sets $K_{0}\left(\psi_{h}\right)$ in $W_{0}^{m, p}(\Omega)$ $(m \geq 1)$ associated to a sequence $\left(\psi_{h}\right)$ of obstacles, and we characterize their Mosco convergence to a set $K_{0}(\psi)$ in terms of the Mosco convergence of $\left(K_{0}\left(\mathbf{1}_{\left\{\psi_{h}>t\right\}}\right)\right)$ to $K_{0}\left(\mathbf{1}_{\{\psi>t\}}\right)$, where $\mathbf{1}_{\left\{\psi_{h}>t\right\}}$ and $\mathbf{1}_{\{\psi>t\}}$ denote the characteristic functions of the level sets $\left\{\psi_{h}>t\right\}$ and $\{\psi>t\}$, respectively, and $t$ varies in a dense subset of $\mathbb{R}$. This result is first achieved in section 3 for a sequence of obstacles which take the value $-\infty$ (i.e. give no constraint) outside a fixed compact subset of $\Omega$ on which they are equi-bounded from above. Next (section 4), these conditions are weakened by means of a proper control on the range of the functions $\psi_{h}$ and on their behaviour near the boundary of $\Omega$.

In a second part (section 5), we give necessary and sufficient conditions for the convergence in $W_{0}^{m, p}(\Omega)$ of the sequence $\left(K_{0}\left(\mathbf{1}_{E_{h}}\right)\right)$ to $K_{0}\left(\mathbf{1}_{E}\right)$, where $E_{h}$ and $E$ are subsets of $\Omega$. Here (Theorem 5.1), the characterization in expressed through the convergence of the capacities of $E_{h} \cap A$ to the capacity of $E \cap A$, where $A$ is taken in a sufficiently large family of open subsets of $\Omega$. In particular, if we specify the sets $E_{h}$ to be the level sets $\left\{\psi_{h}>t\right\}$ of a sequence $\left(\psi_{h}\right)$ of obstacles, then we obtain the main result of 
the paper: the characterization of the convergence of $\left(K_{0}\left(\psi_{h}\right)\right)$ to $K_{0}(\psi)$ in terms of convergence of capacities of level sets (Theorem 5.12). The results in section 5 are confined to the case $p=2$.

Finally, we want to point out that the main difficulty in the attainment of these results is the loss of the lattice structure passing from $W_{0}^{1, p}(\Omega)$ to $W_{0}^{m, p}(\Omega)(m>1)$. This is overcome making use chiefly of suitable approximation lemmas for $C_{m, p}$-quasi continuous functions, and of the representation of the elements in $W^{m, p}\left(\mathbb{R}^{n}\right)$ as a convolution between an $L^{p}\left(\mathbb{R}^{n}\right)$ function and a non-negative kernel (the Bessel kernel).

\section{Notations and preliminaries}

Throughout this paper $n$ and $m$ are positive integers, $p$ a real number with $1<p<+\infty$ and $\Omega$ an open subset of $\mathbb{R}^{n}$ (not necessarily bounded). We denote by $W^{m, p}(\Omega)$ the space of functions $u \in L^{p}(\Omega)$ whose distributional derivatives $D^{\alpha} u$ are in $L^{p}(\Omega)$ for every multi-index $\alpha$ with $|\alpha| \leq m$. We equip $W^{m, p}(\Omega)$ with the usual norm

$$
\|u\|_{m, p ; \Omega}=\left(\sum_{|\alpha| \leq m}\left\|D^{\alpha} u\right\|_{L^{p}(\Omega)}^{p}\right)^{\frac{1}{p}} .
$$

$W_{0}^{m, p}(\Omega)$ will stand for the closure of $C_{0}^{\infty}(\Omega)$ in $W^{m, p}(\Omega)$. Moreover we set $H^{m}(\Omega)=W^{m, 2}(\Omega)$ and $H_{0}^{m}(\Omega)=W_{0}^{m, 2}(\Omega)$. If $u \in W^{m, p}\left(\mathbb{R}^{n}\right)$, by $u \in W_{0}^{m, p}(\Omega)$ we mean that the restriction $u \Upsilon_{\Omega}$ of $u$ to $\Omega$ is in $W_{0}^{m, p}(\Omega)$.

We denote by $\mathcal{A}(\Omega)$ the class of all open subsets of $\Omega$, and by $\mathcal{B}(\Omega)$ the $\sigma$-field of all Borel subsets of $\Omega$. If $A$ and $B$ are subsets of $\mathbb{R}^{n}$, we write $A \Subset B$ if the closure $\bar{A}$ is compact and contained in $B$. A family $\left(A_{t}\right)_{t \in \mathbb{R}}$ of elements of $\mathcal{A}(\Omega)$ is said to be a chain in $\mathcal{A}(\Omega)$ if $A_{s} \Subset A_{t}$ whenever $s, t \in \mathbb{R}$ and $s<t$. We say (see [13], [17]) that a subset $\mathcal{R}$ of $\mathcal{A}(\Omega)$ is:

(a) cofinal in $\Omega$, if for every subset $B$ of $\Omega$ with $B \Subset \Omega$ there exists $A \in \mathcal{R}$ with $B \Subset A$;

(b) dense in $\mathcal{A}(\Omega)$, if for every pair $A_{1}, A_{2}$ in $\mathcal{A}(\Omega)$, with $A_{1} \Subset A_{2}$, there exists $A \in \mathcal{R}$ with $A_{1} \Subset A \Subset A_{2}$;

(c) rich in $\mathcal{A}(\Omega)$, if for every chain $\left(A_{t}\right)_{t \in \mathbb{R}}$ in $\mathcal{A}(\Omega)$ the set $\{t \in \mathbb{R} \mid$ $\left.A_{t} \notin \mathcal{R}\right\}$ is at most countable. 
It is easy to see that every rich set is dense and that every dense set is cofinal. Moreover the intersection of a countable family of rich sets is rich.

$L^{p}, W^{m, p}, H^{m},\|\cdot\|_{m, p}, \mathcal{A}$ and $\mathcal{B}$ will stand for $L^{p}\left(\mathbb{R}^{n}\right), W^{m, p}\left(\mathbb{R}^{n}\right)$, $H^{m}\left(\mathbb{R}^{n}\right),\|\cdot\|_{m, p ; \mathbb{R}^{n}}, \mathcal{A}\left(\mathbb{R}^{n}\right)$ and $\mathcal{B}\left(\mathbb{R}^{n}\right)$, respectively.

For every $s, t \in \mathbb{R}$ we set

$$
s \wedge t=\min \{s, t\}, \quad s \vee t=\max \{s, t\}, \quad s^{+}=s \vee 0 .
$$

By $\mathbb{R}^{+}$and $\mathbb{R}^{-}$we indicate the sets $\{t \in \mathbb{R} \mid t \geq 0\}$ and $\{t \in \mathbb{R} \mid t \leq 0\}$, respectively.

For every subset $E$ of $\mathbb{R}^{n}$ we denote by $\mathbf{1}_{E}$ the characteristic function of $E$ with respect to $\mathbb{R}^{n}$, defined by $\mathbf{1}_{E}(x)=1$ if $x \in E$ and $\mathbf{1}_{E}(x)=0$ if $x \in \mathbb{R}^{n} \backslash E$. Moreover we set $\chi_{E}(x)=0$ if $x \in E$ and $\chi_{E}(x)=-\infty$ if $x \in \mathbb{R}^{n} \backslash E$.

The abbreviation a.e. will mean almost everywhere with respect to the Lebesgue measure.

Recall now some notions we shall need in the subsequent sections.

\subsection{Bessel potentials and Sobolev spaces}

We define a linear map $J_{m}: L^{p} \rightarrow L^{p}$ by setting for every $f \in L^{p}$ and for every $x \in \mathbb{R}^{n}$ (see, for example, [26] chapter $\mathrm{V}$ ):

$$
\left(J_{m} f\right)(x)=\left(G_{m} \star f\right)(x)=\int_{\mathbb{R}^{n}} G_{m}(x-y) f(y) \mathrm{d} y,
$$

where $G_{m}$ is the $L^{1}$ function on $\mathbb{R}^{n}$ whose Fourier transform, defined through

$$
\widehat{G}_{m}(\xi)=\int_{\mathbb{R}^{n}} \mathrm{e}^{2 \pi i \xi \cdot x} G_{m}(x) \mathrm{d} x
$$

for every $\xi \in \mathbb{R}^{n}$, is given by $\left(1+4 \pi^{2}|\xi|^{2}\right)^{-m / 2}$. It is known that $G_{m} \geq 0$ and $\left\|G_{m}\right\|_{L^{1}}=1$. The function $J_{m} f$ is called the $L^{p}$-Bessel potential of $f$ of order $m$.

ThEOREM 1.1 ([26] chapter V, theorem 3). - $J_{m}$ establishes a bijective correspondence between $L^{p}$ and $W^{m, p}$. Moreover there exist two positive constants $c_{1}$ and $c_{2}$ such that for every $f \in L^{p}$

$$
c_{1}\|f\|_{L^{p}} \leq\left\|J_{m} f\right\|_{m, p} \leq c_{2}\|f\|_{L^{p}} .
$$

Hence $J_{m}$ is an isomorphism of Banach spaces between $L^{p}$ and $W^{m, p}$. 
The spaces $\mathcal{L}_{m}^{p}=J_{m}\left(L^{p}\right)$ of all $L^{p}$-Bessel potentials of order $m$ coincides with $W^{m, p}$. It will be equipped with the norm induced by $J_{m}$

$$
\|u \mid\|_{m, p}=\|u\|_{\mathcal{L}_{m}^{p}}=\left\|\left(J_{m}\right)^{-1} u\right\|_{L^{p}}
$$

which turns out to be equivalent to the norm $\|\cdot\|_{m, p}$ introduced in (1.1).

Remark 1.2. - For every $u \in W^{m, p}$ there exists a function $v \in W^{m, p}$ with $v \geq u^{+}$and $\|v\|_{m, p} \leq\|u\|_{m, p}$. Indeed, if $f=\left(J_{m}\right)^{-1} u$, then it suffices to define $v=J_{m} f^{+}$.

\subsection{Capacities and quasi-topology}

If $K$ is a compact subset of $\mathbb{R}^{n}$, we define

$$
\begin{aligned}
& C_{m, p}(K)=\inf \left\{\|\varphi\|_{m, p}^{p} \mid \varphi \in C_{0}^{\infty}\left(\mathbb{R}^{n}\right), \varphi \geq \mathbf{1}_{K} \text { on } \mathbb{R}^{n}\right\} \\
& B_{m, p}(K)=\inf \left\{\|\varphi\|_{m, p}^{p} \mid \varphi \in C_{0}^{\infty}\left(\mathbb{R}^{n}\right), \varphi \geq \mathbf{1}_{K} \text { on } \mathbb{R}^{n}\right\} .
\end{aligned}
$$

We extend $C_{m, p}$ and $B_{m, p}$ to all the subsets of $\mathbb{R}^{n}$ as external capacities in the usual way (see, for example, [7]). The equivalence of $\|\cdot\|_{m, p}$ and \|\|$\cdot \|_{m, p}$ implies that $C_{m, p}$ and $B_{m, p}$ are equivalent capacities; thus the notions involving sets of zero or arbitrarily small capacity are the same for both of them. In such cases the symbol "cap" will be often used instead of $C_{m, p}$ or $B_{m, p}$.

Given a subset $E$ of $\mathbb{R}^{n}$, if a statement depending on $x \in \mathbb{R}^{n}$ holds for every $x \in E$ except for a set $N \subseteq E$ with $\operatorname{cap}(N)=0$, then we say that it holds $(m, p)$-quasi everywhere $((m, p)$-q.e.) on $E$ (we usually omit $E$ if it is $\left.\mathbb{R}^{n}\right)$.

We say that a function $f: E \rightarrow \mathbb{R}$ is $(m, p)$-quasi continuous if for every $\epsilon>0$ there exists an open subset $A$ of $\mathbb{R}^{n}$ with $\operatorname{cap}(A)<\epsilon$, such that $f\left\lceil_{E \backslash A}\right.$ is continuous on $E \backslash A$. The definition of $(m, p)$-quasi lower semicontinuous (l.s.c.) and ( $m, p)$-quasi upper semicontinuous (u.s.c.) function is given in a similar way.

$B_{m, p}$ turns out to coincide with the capacity defined in [20], paragraph 8 , and in [21] by means of the Bessel kernel $G_{m}$. Hence we have, in particular, that every function $u \in W^{m, p}$ has an $(m, p)$-quasi continuous representative, denoted by $\tilde{u}$, which is unique up to $(m, p)$-q.e. equivalence. Indeed (lemma 5.8 in [20]), if $f$ and $g$ are $(m, p)$-quasi continuous and $f \geq g$ a.e., then 
$f \geq g(m, p)$-q.e. If $u \in W_{0}^{m, p}(\Omega)$, the restriction to $\Omega$ of the $(m, p)$-quasi continuous representative of any extension of $u$ to a function of $W^{m, p}$ is well defined and denoted by $\tilde{u}$.

It can be proved that for every $f \in L^{p}$ the function $G_{m} \star f$ is $(m, p)$-quasi continuous. Moreover, for any $E \subseteq \mathbb{R}^{n}$

$$
\begin{aligned}
B_{m, p}(E) & =\inf \left\{\|f\|_{L^{p}}^{p} \mid f \in L^{p}, f \geq 0 \text { on } \mathbb{R}^{n}, G_{m} \star f \geq 1 \text { on } E\right\} \\
& =\inf \left\{\|u\|_{m, p}^{p} \mid u \in W^{m, p}, \tilde{u} \geq \mathbf{1}_{E}(m, p) \text {-q.e. }\right\} \\
& =\inf \left\{\|u\|_{m, p}^{p} \mid u \in W^{m, p}, \tilde{u} \geq 1(m, p) \text {-q.e. on } E\right\} .
\end{aligned}
$$

As for $C_{m, p}$ we have

$$
C_{m, p}(E)=\inf \left\{\|u\|_{m, p}^{p} \mid u \in W^{m, p}, \tilde{u} \geq \mathbf{1}_{E}(m, p) \text {-q.e. }\right\} .
$$

We point out that $B_{m, p}$ is countably subadditive.

We say that a sequence $\left(f_{h}\right)$ of functions from $\mathbb{R}^{n}$ into $\mathbb{R}$ converges in $(m, p)$-capacity to a function $f$ from $\mathbb{R}^{n}$ into $\mathbb{R}$, if for every $\epsilon>0$

$$
\lim _{h \rightarrow+\infty} \operatorname{cap}\left\{x \in \mathbb{R}^{n}|| f_{h}(x)-f(x) \mid \geq \epsilon\right\}=0 .
$$

Proposition 1.3 ([21], theorem 4). - If $\left(u_{h}\right)$ is a sequence in $W^{m, p}$ which converges to $u$ in $W^{m, p}$, then $\left(\tilde{u}_{h}\right)$ converge to $\tilde{u}$ in $(m, p)$-capacity and there exists a subsequence $\left(\tilde{u}_{h_{k}}\right)$ of $\left(\tilde{u}_{h}\right)$ which converges to $\tilde{u}(m, p)$-q.e.

Proposition 1.4. - If $\left(f_{h}\right)$ and $\left(g_{h}\right)$ are sequences of functions from $\mathbb{R}^{n}$ into $\mathbb{R}$ which converge in $(m, p)$-capacity to the functions $f$ and $g$, respectively, then the sequences $\left(f_{h} \wedge g_{h}\right)$ and $\left(f_{h} \vee g_{h}\right)$ converge in $(m, p)$ capacity to $f \wedge g$ and $f \vee g$, respectively.

Proof. - Apply the inequality

$$
\left|f_{h} \wedge g_{h}-f \wedge g\right| \leq\left|f_{h}-f\right|+\left|g_{h}-g\right|
$$

and the analogous one for $\vee$.

We shall use some other topological notions connected with capacities; to handle them it is more convenient to deal with countably subadditive capacities, though most of the results still hold for equivalent capacities. We refer to [17] for a systematic account. 


\section{Enrico Vitali}

Let $E, E_{1}$ and $E_{2}$ be subsets of $\mathbb{R}^{n}$. We say that $E_{1}$ is $(m, p)$-quasi contained in $E_{2}$ if $\operatorname{cap}\left(E_{1} \backslash E_{2}\right)=0$; consequently, we say that $E_{1}$ is $(m, p)$ equivalent to $E_{2}$ if $\operatorname{cap}\left(E_{1} \triangle E_{2}\right)=0$ (where $\triangle$ denotes the symmetric difference).

$E$ is said to be $(m, p)$-quasi open (resp. quasi closed, quasi compact, quasi Borel) if for every $\epsilon>0$ there exists an open (resp. closed, compact, Borel) set $A$ such that $\operatorname{cap}(E \triangle A)<\epsilon$.

It is easy to see that $A$ is $(m, p)$-quasi open if and only if $\mathbb{R}^{n} \backslash A$ is $(m, p)$-quasi closed, and that any countable union or finite intersection of $(m, p)$-quasi open sets is $(m, p)$-quasi open. Moreover $A$ is $(m, p)$-quasi open (resp. quasi closed) if and only if $1_{E}$, or equivalently $\chi_{E}$, is $(m, p)$-quasi l.s.c. (resp. quasi u.s.c.).

It can be proved that a function $f: \mathbb{R}^{n} \rightarrow \mathbb{R}$ is $(m, p)$-quasi l.s.c. (quasi u.s.c.) if and only if the sets $\left\{x \in \mathbb{R}^{n} \mid f(x)>t\right\}$ (resp. $\left\{x \in \mathbb{R}^{n} \mid f(x) \geq t\right\}$ ) are $(m, p)$-quasi open (resp. quasi closed) for every $t \in \mathbb{R}$.

Let $u \in W^{m, p}$ and $t>0$; by approximating $u$ by $C_{0}^{\infty}\left(\mathbb{R}^{n}\right)$ functions it is easy to see that the set $\left\{x \in \mathbb{R}^{n} \mid \tilde{u}(x) \geq t\right\}$ is $(m, p)$-quasi compact. It follows that an $(m, p)$-quasi closed set with finite capacity is $(m, p)$-quasi compact.

It can be shown ([17], theorem 2.8) that for every subset $E$ of $\mathbb{R}^{n}$ there is an $(m, p)$-quasi closed set $\widetilde{E}$ which is $(m, p)$-quasi contained in every $(m, p)$ quasi closed set which $(m, p)$-quasi contains $E ; \widetilde{E}$ is unique up to sets of null capacity and it will be referred to as the $(m, p)$-quasi closure of $E$.

We will sometimes drop the prefix $(m, p)$ in the foregoing notations.

Proposition 1.5. - Let $E$ and $Z$ be subsets of $\mathbb{R}^{n}$. Then the following conditions are equivalent:

(a) every $(m, p)$-quasi open set $G$ with $\operatorname{cap}(G \cap Z)>0$ is such that $\operatorname{cap}(G \cap E)>0 ;$

(b) $Z$ is $(m, p)$-quasi contained in $\tilde{E}$.

\section{Proof}

(a) $\Rightarrow$ (b) Consider the quasi open set $G=\mathbb{R}^{n} \backslash \widetilde{E}$. Since $\operatorname{cap}(G \cap E)=$ $\operatorname{cap}(E \backslash \widetilde{E})=0$, we have, by condition $(\mathrm{a}), \operatorname{cap}(Z \backslash \tilde{E})=\operatorname{cap}(G \cap Z)=0$, i.e. $Z$ is quasi contained in $\widetilde{E}$.

(b) $\Rightarrow$ (a) Fix a quasi open set $G$; let us assume that $\operatorname{cap}(G \cap E)=0$ and prove that $\operatorname{cap}(G \cap Z)=0$. Consider the quasi closed set $F=\widetilde{E} \backslash G$. 
Since $E \backslash F$ is $(m, p)$-equivalent to $E \cap G$, we have $\operatorname{cap}(E \backslash F)=0$, i.e. $E$ is quasi contained in $F$. Hence $\widetilde{E}$ is quasi contained in $F$, thus $F$ is $(m, p)$-equivalent to $\widetilde{E}$. This means that $\operatorname{cap}(G \cap \widetilde{E})=0$ and therefore $\operatorname{cap}(G \cap Z)=0$, as $G \cap Z$ is quasi contained in $G \cap \tilde{E}$ by assumption.

Finally, for any function $\varphi: \mathbb{R}^{n} \rightarrow[0,+\infty]$ we define

$$
B_{m, p}(\varphi)=\inf \left\{\|u\|_{m, p}^{p} \mid u \in W^{m, p}, \varphi \leq \tilde{u}(m, p) \text {-q.e. }\right\} .
$$

\subsection{Convergence in the sense of Mosco}

Let $V$ be a Banach space with norm $\|\cdot\|$.

DEFINITION 1.6. - Let $\left(K_{h}\right)$ be a sequence of subsets of $V$.

(i) The strong lower limit of the sequence $\left(K_{h}\right)$ in $V$ is the set $\mathrm{s}$-liminf $K_{h}$ of all $u \in V$ with the following property: there exist an index $k \in \mathbb{N}$ and a sequence $\left(u_{h}\right)$ converging to $u$ strongly in $V$ such that $u_{h} \in K_{h}$ for every $h \geq k$.

(ii) The weak upper limit of the sequence $\left(K_{h}\right)$ in $V$ is the set $\mathrm{w}-\lim \sup K_{h}$ of all $u \in V$ with the following property: there exist a $h \rightarrow+\infty$

sequence $\left(u_{k}\right)$ converging to $u$ weakly in $V$ and a subsequence $\left(K_{h_{k}}\right)$ of $\left(K_{h}\right)$ such that $u_{k} \in K_{h_{k}}$ for every $k \in \mathbb{N}$.

Note that $\operatorname{s-liminf}_{h \rightarrow+\infty} K_{h} \subseteq \underset{h \rightarrow+\infty}{\text { w-lim sup }} K_{h}$.

Definition 1.7. - Let $\left(K_{h}\right)$ be a sequence of subsets of $V$ and let $K$ be a subset of $V$. We say that $\left(K_{h}\right)$ converges to $K$ in the sense of Mosco in $V$ (see [22]), and we write

$$
K_{h} \rightarrow K \quad \text { in } V \text {, }
$$

if $\underset{h \rightarrow+\infty}{\liminf } K_{h}=\underset{h \rightarrow+\infty}{\mathrm{w}-\limsup _{h \rightarrow+\infty}} K_{h}=K$.

Remark 1.8. - $\underset{h \rightarrow+\infty}{\mathrm{s}-\liminf _{h}} K_{h}$ and $\mathrm{w}-\limsup _{h \rightarrow+\infty} K_{h}$ are the lower and upper limits of the sequence $\left(K_{h}\right)$, in the sense of Kuratowski, taken with respect to the strong and, respectively, to the sequential weak convergence in $V$ (see for instance [22]; [23] and [3] for a more general treatment of these concepts). 


\section{Enrico Vitali}

An important general property of the strong lower limit is that it is strongly closed in $V$. Moreover we shall need the following result.

Proposition 1.9 ([22], theorem B). - Assume that $V$ is reflexive; let $\left(K_{h}\right)$ be a sequence of convex subsets of $V$ which converges to a subset $K$ of $V$ in the sense of Mosco (in $V$ ). Then

$$
\lim _{h \rightarrow+\infty} \inf _{u \in K_{h}}\|u\|=\inf _{u \in K}\|u\|
$$

In the sequel we shall take as $V$ one of the spaces $W^{m, p}, W_{0}^{m, p}(\Omega)$ or $L^{p}\left(B_{m, p}\right)$ (see definition 2.15).

DEFINITION 1.10. - Given a function $\psi$ from $\mathbb{R}^{n}$ into $\overline{\mathbb{R}}$, for every subset $B$ of $\mathbb{R}^{n}$ we define

$$
K(\psi, B)=\left\{u \in W^{m, p} \mid \tilde{u} \geq \psi(m, p)-q . e \text {. on } B\right\}
$$

while, if $B \subseteq \Omega$,

$$
K_{0}(\psi, B)=\left\{u \in W_{0}^{m, p}(\Omega) \mid \tilde{u} \geq \psi(m, p) \text {-q.e. on } B\right\} .
$$

By proposition 1.3, $K(\psi, B)$ is a closed convex subset of $W^{m, p}$ and $K_{0}(\psi, B)$ a closed convex subset of $W_{0}^{m, p}(\Omega)$. We shall denote $K\left(\psi, \mathbb{R}^{n}\right)$ and $K_{0}(\psi, \Omega)$ by $K(\psi)$ and $K_{0}(\psi)$, respectively; note that $K(\psi, B)$ is nothing but $K\left(T_{B} \psi\right)$, where

$$
T_{B} \psi= \begin{cases}\psi & \text { on } B \\ -\infty & \text { on } \mathbb{R}^{n} \backslash B\end{cases}
$$

If $\left(\psi_{h}\right)$ is a sequence of functions from $\mathbb{R}^{n}$ into $\mathbb{\mathbb { R }}$ and $B$ a subset of $\mathbb{R}^{n}$, we shall use the notation

$$
\begin{aligned}
& K^{\prime}\left(\left(\psi_{h}\right), B\right)=\mathrm{s}-\liminf _{h \rightarrow+\infty} K\left(\psi_{h}, B\right) \quad\left(\text { in } W^{m, p}\right) \\
& K^{\prime \prime}\left(\left(\psi_{h}\right), B\right)=\underset{h \rightarrow+\infty}{\mathrm{w}-\lim \sup } K\left(\psi_{h}, B\right) \quad\left(\text { in } W^{m, p}\right) \text {; }
\end{aligned}
$$

if $B=\mathbb{R}^{n}$ we shall simply write $K^{\prime}\left(\left(\psi_{h}\right)\right)$ and $K^{\prime \prime}\left(\left(\psi_{h}\right)\right)$, respectively. $K_{0}^{\prime}\left(\left(\psi_{h}\right), B\right), K_{0}^{\prime \prime}\left(\left(\psi_{h}\right), B\right)$ (for $\left.B \subseteq \Omega\right), K_{0}^{\prime}\left(\left(\psi_{h}\right)\right)$ and $K_{0}^{\prime \prime}\left(\left(\psi_{h}\right)\right)$ are defined in the obvious way. 


\section{Approximation lemmas and convex sets}

In this section we first state two approximation results (lemmas 2.1 and 2.3 ), which will be widely used. Next we define the class of closed unilateral convex sets in $W_{0}^{m, p}(\Omega)$ and prove that it coincides with the class of convex sets of obstacle type in $W_{0}^{m, p}(\Omega)$ (proposition 2.9). This generalizes the analogous result given in [4] (theorem 3.2) for the case $m=1$. Moreover, we show the closedness of the class with respect to the convergence in the sense of Mosco in $W_{0}^{m, p}(\Omega)$ (proposition 2.12). Finally we point out a useful connection (proposition 2.19) between the Mosco convergence for convex sets of obstacle type in $W^{m, p}$ and in the space $L^{p}\left(B_{m, p}\right)$ (see definition 2.15).

Let us recall a result from [9] (lemma 1.5):

Lemma 2.1. - Let $f: \mathbb{R}^{n} \rightarrow \mathbb{R}$ be an (m,p)-quasi u.s.c. function. Assume that there exists a function $w \in W^{m, p}$ with $\tilde{w} \geq f(m, p)$-q.e. Then there exists a sequence $\left(u_{h}\right)$ in $W^{m, p}$ such that $\left(\tilde{u}_{h}\right)$ decreases and converges to $f(m, p)$-q.e.

Before giving the other fundamental approximation result, we need a theorem from non-linear potential theory (see theorem 3.2 in [1]).

TheOrem 2.2. - There exists a constant $c$ depending only on $n, p$ and $m$ such that

$$
\frac{1}{4} B_{m, p}(\varphi) \leq \int_{0}^{+\infty} B_{m, p}\left(\left\{x \in \mathbb{R}^{n} \mid \varphi(x)>t\right\}\right) \mathrm{d} t^{p} \leq c B_{m, p}(\varphi)
$$

for every function $\varphi: \mathbb{R}^{n} \rightarrow[0,+\infty]$ (here $\mathrm{d} t^{p}$ means $p t^{p-1} \mathrm{~d} t$ ).

LEMma 2.3. - Let $\left(f_{h}\right)$ be a sequence of functions from $\mathbb{R}^{n}$ into $\mathbb{R}$ and let $f$ be a function from $\mathbb{R}^{n}$ into $\mathbb{R}$. Then the following statements hold:

(i) let $f_{h}$ be $(m, p)$-quasi u.s.c. for every $h \in \mathbb{N}$. If $\left(f_{h}\right)$ is decreasing and converges to zero $(m, p)-q . e$. , and there exists a function $w \in$ $W^{m, p}$ such that $\tilde{w} \geq f_{1}(m, p)$-q.e., then $\left(f_{h}\right)$ converges to zero in $(m, p)$-capacity;

(ii) if $\left(f_{h}\right)$ converges to zero in $(m, p)$-capacity and there exists a sequence $\left(w_{h}\right)$, which converges strongly in $W^{m, p}$, such that $f_{h} \leq \tilde{w}_{h}$ $(m, p)$-q.e. for every $h \in \mathbb{N}$, then $\left(B_{m, p}\left(f_{h}^{+}\right)\right)$converges to zero; 
(iii) if $u$ is a function in $W^{m, p}$ and $\left(B_{m, p}\left(\left(f_{h}-\tilde{u}\right)^{+}\right)\right)$converges to zero, then we can find a sequence $\left(u_{h}\right)$ in $W^{m, p}$ which converges to $u$ strongly in $W^{m, p}$ and for which $\tilde{u}_{h} \geq f_{h}(m, p)$-q.e. for every $h \in \mathbb{N}$.

Proof

(i) For every $h \in \mathbb{N}$ and $t>0$ the sets $\left\{f_{h} \geq t\right\}=\left\{x \in \mathbb{R}^{n} \mid f_{h}(x) \geq t\right\}$ are quasi closed and have finite capacity, hence they are quasi compact. The continuity of $B_{m, p}$ on decreasing sequences of quasi compact sets (see theorem 2.10 in [17]) yields that $\left(B_{m, p}\left(\left\{f_{h} \geq t\right\}\right)\right)$ converges to zero.

(ii) Let $w$ be the limit of $\left(w_{h}\right)$ in $W^{m, p}$. For every $h \in \mathbb{N}$ and $t>0$ the subadditivity of $B_{m, p}$ gives:

$$
\begin{aligned}
B_{m, p}\left(\left\{f_{h} \geq t\right\}\right) & \leq B_{m, p}\left(\left\{\tilde{w}_{h} \geq t\right\}\right) \\
& \leq B_{m, p}\left(\left\{\tilde{w} \geq \frac{t}{2}\right\}\right)+B_{m, p}\left(\left\{\tilde{w}_{h}-\tilde{w} \geq \frac{t}{2}\right\}\right) .
\end{aligned}
$$

On account of remark 1.2 , from theorem 2.2 we easily obtain that

$$
\begin{gathered}
\int_{0}^{+\infty} B_{m, p}\left(\left\{\tilde{w} \geq \frac{t}{2}\right\}\right) \mathrm{d} t^{p}<+\infty, \\
\lim _{h \rightarrow+\infty} \int_{0}^{\infty} B_{m, p}\left(\left\{\tilde{w}_{h}-\tilde{w} \geq \frac{t}{2}\right\}\right) \mathrm{d} t^{p}=0 .
\end{gathered}
$$

Since $\left(B_{m, p}\left(\left\{f_{h} \geq t\right\}\right)\right)$ converges to zero for every $t>0$, we can apply a version of the Lebesgue dominated convergence theorem with respect to the measure $\mathrm{d} t^{p}$, so that

$$
\lim _{h \rightarrow+\infty} \int_{0}^{+\infty} B_{m, p}\left(\left\{f_{h} \geq t\right\}\right) \mathrm{d} t^{p}=0 .
$$

Now we conclude by theorem 2.2 .

(iii) is an easy consequence of the definition of $B_{m, p}$ on non-negative functions.

Remark 2.4. - lemma 2.3 is a slightly extended version of lemma 1.6 in [8], and in fact it can also be directly proved by a similar argument. 
Definition 2.5. - We say that $K_{0}$ is a unilateral convex set in $W_{0}^{m, p}(\Omega)$ if $K_{0}$ is a subset of $W_{0}^{m, p}(\Omega)$ with the following property: if $u, v \in K_{0}, w \in W_{0}^{m, p}(\Omega)$ and $w \geq u \wedge v$ a.e. in $\Omega$, then $w \in K_{0}$.

We point out that $K_{0}$ immediately turns out to be convex.

Definition 2.6. - We say that a subset $K_{0}$ of $W_{0}^{m, p}(\Omega)$ is $C^{\infty}$-convex when the following condition holds: if $u, v \in K_{0}$ and $\varphi$ is a function in $C^{\infty}(\Omega)$ with values in $[0,1]$ and bounded derivatives of every order, then $\varphi u+(1-\varphi) v \in K_{0}$.

It is clear that the unilaterality condition implies $C^{\infty}$-convexity. We now show that actually the two conditions are equivalent for every closed unilateral convex set $K_{0}$ provided it is stable under addition of non-negative $W_{0}^{m, p}(\Omega)$ functions, i.e. such that

$$
\text { if } u \in K_{0}, v \in W_{0}^{m, p}(\Omega) \text { and } v \geq u \text { a.e. in } \Omega \text {, then } v \in K_{0} \text {; }
$$

If $K_{0}$ is a subset of $W_{0}^{m, p}(\Omega)$, we put

$$
\mathcal{K}\left(K_{0}\right)=\bigcap_{\omega \Subset \Omega}\left\{u \in W^{m, p} \mid \exists v \in K_{0} u=v \text { a.e. on } \omega\right\}
$$

where the intersection runs over all the open sets $\omega \Subset \Omega$.

LEMMA 2.7. - Let $K_{0}$ be a $C^{\infty}$-convex set in $W_{0}^{m, p}(\Omega)$, closed in $W_{0}^{m, p}(\Omega)$ and satisfying condition $(\alpha)$ introduced above. Then $\mathcal{K}\left(K_{0}\right)$ is a $C^{\infty}$-convex set in $W^{m, p}$, closed in $W^{m, p}$, for which $(\alpha)$ holds and

$$
K_{0}=\mathcal{K}\left\lceil\Omega\left(K_{0}\right) \cap W_{0}^{m, p}(\Omega),\right.
$$

where $\mathcal{K}_{\uparrow_{\Omega}}\left(K_{0}\right)=\left\{u \uparrow_{\Omega} \mid u \in \mathcal{K}\left(K_{0}\right)\right\}$. Moreover, if $K_{0}=K_{0}(\psi)$ then $\mathcal{K}\left(K_{0}\right)=K\left(T_{\Omega} \psi\right)$ (where $T_{\Omega}$ is defined in (1.3)).

Proof. - Assume $K_{0} \neq \emptyset$, otherwise there is nothing to prove; then we can suppose $0 \in K_{0}$.

By $C^{\infty}$-convexity, if $u \in K_{0}$ and $\varphi$ is a $C_{0}^{\infty}\left(\mathbb{R}^{n}\right)$ function taking its values in $[0,1]$, then $\varphi u \in K_{0}$. The closedness of $\mathcal{K}\left(K_{0}\right)$ now follows easily by means of standard cut-off functions, as well as the equality $\mathcal{K}\left(K_{0}\right)=K\left(T_{\Omega} \psi\right)$ in the case $K_{0}=K_{0}(\psi) . C^{\infty}$-convexity of $\mathcal{K}\left(K_{0}\right)$ is immediate. Les us check condition $(\alpha)$. Consider $u \in \mathcal{K}\left(K_{0}\right)$ and $v \in W^{m, p}$ 
with $v \geq u$ a.e.; we fix two open sets $\omega$ and $\omega^{\prime}$ such that $\omega \Subset \omega^{\prime} \Subset \Omega$, and a function $\varphi \in C_{0}^{\infty}\left(\omega^{\prime}\right)$ with $\varphi=1$ on $\omega$ and $0 \leq \varphi \leq 1$. Since $u \in \mathcal{K}\left(K_{0}\right)$, there exists a function $u_{0} \in K_{0}$ equal to $u$ a.e. on $\omega^{\prime}$, thus $\varphi v \geq \varphi u_{0}$ a.e. in $\Omega$ and $\varphi u_{0} \in K_{0}$; by condition $(\alpha)$ which holds for $K_{0}, \varphi v \in K_{0}$. Moreover, $v=\varphi v$ a.e. on $\omega$ and then $v \in \mathcal{K}\left(K_{0}\right)$ because of the arbitrary choice of $\omega$.

Let us prove (2.1); only the inclusion $K_{0} \supseteq \mathcal{K} \uparrow \Omega\left(K_{0}\right) \cap W_{0}^{m, p}(\Omega)$ is not trivial. Let $u \in \mathcal{K}\left(K_{0}\right)$ with $u\left\lceil\Omega \in W_{0}^{m, p}(\Omega)\right.$. Fix $\epsilon>0$; since $u \uparrow_{\Omega} \in W_{0}^{m, p}(\Omega)$ there exists a function $\eta \in C_{0}^{\infty}(\Omega)$ such that $0 \leq \eta \leq 1$ and $\|u-\eta u\|_{m, p ; \Omega} \leq \epsilon$ (see theorem 3.1 in [18] and theorem 5 in [19]). Let $\omega \Subset \Omega$ be an open set containing spt $\eta$; since $u \in \mathcal{K}\left(K_{0}\right)$, and hence $\eta u \in \mathcal{K}\left(K_{0}\right)$, we can find a function $w_{0} \in K_{0}$ equal to $\eta u$ a.e. on $\omega$. Consider a function $\varphi \in C_{0}^{\infty}(\omega)$ with $\varphi=1$ on spt $\eta$ and $0 \leq \varphi \leq 1$; we have $\eta u=\varphi(\eta u)=\varphi w_{0}$ a.e. on $\Omega$ and $\varphi w_{0} \in K_{0}$. Since $\epsilon>0$ is arbitrary and $K_{0}$ is closed in $W_{0}^{m, p}(\Omega)$, we conclude that $u \in K_{0}$, therefore $\mathcal{K} \uparrow_{\Omega}\left(K_{0}\right) \cap W_{0}^{m, p}(\Omega) \subseteq K_{0}$.

Proposition 2.8. - Let $K_{0}$ be a closed subset of $W_{0}^{m, p}(\Omega)$ satisfying condition $(\alpha)$ above. Then $K_{0}$ is a unilateral convex set if and only if $K_{0}$ is $C^{\infty}$-convex.

Proof. - Assume that $K_{0}$ is $C^{\infty}$-convex and let us prove that it is a unilateral convex set.

(i) Case $\Omega=\mathbb{R}^{n}$. Let $u, v \in K_{0}, w \in W^{m, p}$ with $w \geq u \wedge v$ a.e. in $\Omega$; we have to prove that $w \in K_{0}$. Fix $h \in \mathbb{N}$; the sets $A_{1}=\{\tilde{u} \geq \tilde{v}+1 / h\}$ and $A_{2}=\{\tilde{v} \geq \tilde{u}+1 / h\}$ are quasi closed and have finite capacity, thus they are quasi compact: we can find compact sets $C_{1}$ and $C_{2}$ and open sets $\omega_{1}$ and $\omega_{2}$ such that

$$
C_{i} \subseteq A_{i} \subseteq C_{i} \cup \omega_{i}, \quad B_{m, p}\left(\omega_{i}\right) \leq \frac{1}{h} \quad(i=1,2)
$$

Let $\varphi_{h}$ be a function in $C_{0}^{\infty}\left(\mathbb{R}^{n}\right)$ with $0 \leq \varphi_{h} \leq 1$ and $\varphi_{h}=0$ on $C_{1}$, $\varphi_{h}=1$ on $C_{2}$. For every $\epsilon>0$, if $2 / h<\epsilon$ the subadditivity of $B_{m, p}$ yields:

$$
B_{m, p}\left(\left\{\left|\varphi_{h} \tilde{u}+\left(1-\varphi_{h}\right) \tilde{v}-\tilde{u} \wedge \tilde{v}\right|>\epsilon\right\}\right) \leq B_{m, p}\left(\omega_{1}\right)+B_{m, p}\left(\omega_{2}\right) \leq \frac{2}{h}
$$

this means that the sequence $\left(\varphi_{h} \tilde{u}+\left(1-\varphi_{h}\right) \tilde{v}\right)$ converges to $\tilde{u} \wedge \tilde{v}$ in $(m, p)$-capacity. For every $h \in \mathbb{N}$ put now $f_{h}=\left(\varphi_{h} \tilde{u}+\left(1-\varphi_{h}\right) \tilde{v}\right) \vee \tilde{w}$; by proposition $1.4\left(f_{h}\right)$ converges to $\tilde{w}$ in $(m, p)$-capacity, therefore, on account of remark 1.2 , we can apply lemma 2.3 and find a sequence $\left(w_{h}\right)$ in $W^{m, p}$ which converges to $w$ strongly in $W^{m, p}$ and with $\tilde{w}_{h} \geq f_{h}(m, p)$-q.e. for 
every $h \in \mathbb{N}$. By $C^{\infty}$-convexity and condition $(\alpha), w_{h} \in K_{0}$ for every $h \in \mathbb{N}$, hence $w \in K_{0}$ because $K_{0}$ is closed in $W^{m, p}$.

(ii) Consider now the general case of $\Omega$ open subset of $\mathbb{R}^{n}$. By lemma 2.7 the set $\mathcal{K}\left(K_{0}\right)$ is closed in $W^{m, p}, C^{\infty}$-convex and satisfies condition $(\alpha)$. Hence we can apply the foregoing part (i) of the proof and get that $\mathcal{K}\left(K_{0}\right)$ is a unilateral convex set in $W^{m, p}$; the same is true for $K_{0}$ in $W_{0}^{m, p}(\Omega)$, as it follows from the equality $K_{0}=\mathcal{K}\left\lceil\Omega\left(K_{0}\right) \cap W_{0}^{m, p}(\Omega)\right.$ given in lemma $2.7 \square$

Let us now show that any closed unilateral convex set is of obstacle type.

Proposition 2.9. - Let $K_{0}$ be a non-empty closed unilateral convex set in $W_{0}^{m, p}(\Omega)$. Then there exists an $(m, p)$-quasi u.s.c. function $\bar{\psi}: \Omega \rightarrow \mathbb{R}$ such that $K_{0}=K_{0}(\bar{\psi}) ; \bar{\psi}$ is uniquely defined on $\Omega$ up to $(m, p)$-q.e. equivalence.

\section{Proof}

(i) Case $\Omega=\mathbb{R}^{n}$. First of all let us prove that for every $k \in \mathbb{N}, k \geq 2$, the following statement holds:

$$
\begin{aligned}
& \text { if } u_{1}, u_{2}, \ldots, u_{k} \in K_{0}, v \in W^{m, p} \\
& \text { and } v \geq u_{1} \wedge u_{2} \wedge \cdots \wedge u_{k} \text { a.e., then } v \in K_{0} .
\end{aligned}
$$

Since $(\beta)_{2}$ holds by assumption, it suffices to show that for every $k \geq 2$ $(\beta)_{k}$ implies $(\beta)_{k+1}$. Fix $k \geq 2$, a subset $\left\{u_{1}, \ldots, u_{k}, u_{k+1}\right\}$ in $K_{0}$ and a function $v \in W^{m, p}$ with $v \geq u_{1} \wedge \cdots \wedge u_{k} \wedge u_{k+1}$ a.e. The function $f=\tilde{u}_{1} \wedge \cdots \wedge \tilde{u}_{k}$ is $(m, p)$-quasi continuous, hence, by lemma 2.1 we can find a sequence $\left(w_{h}\right)$ in $W^{m, p}$ such that $\left(\tilde{w}_{h}\right)$ decreases and converges to $f(m, p)$-q.e. Observe that by assumption $(\beta)_{k}, w_{h} \in K_{0}$ for every $h \in \mathbb{N}$. The sequence $\left(\left(\tilde{w}_{h} \wedge \tilde{u}_{k+1}\right) \vee \tilde{v}\right)_{h}$ decreases and converges $(m, p)$-q.e. to $\tilde{v}$ : by remark 1.2 and lemma 2.3 there exists a sequence $\left(v_{h}\right)$ in $W^{m, p}$ which converges to $v$ in $W^{m, p}$ and for which $\tilde{v}_{h} \geq\left(\tilde{w}_{h} \wedge \tilde{u}_{k+1}\right) \vee \tilde{v}(m, p)$-q.e. for every $h \in \mathbb{N}$. By definition of unilateral convex set, $v_{h} \in K_{0}$ (since $w_{h} \in K_{0}$ ) for every $h \in \mathbb{N}$; the closedness of $K_{0}$ in $W^{m, p}$ implies that $v \in K_{0}$ and thus we have proved that $(\beta)_{k+1}$ holds.

Since $W^{m, p}$ is a separable metric space, there exists a sequence $\left(v_{h}\right)$ of $K_{0}$ dense in $K_{0}$. For every $h \in \mathbb{N}$ let $\psi_{h}=\inf _{1 \leq i \leq h} \widetilde{v}_{i}\left(\psi_{h}\right.$ is defined up to sets of zero $(m, p)$-capacity). The sequence $\left(\psi_{h}\right)$ is decreasing and its infimum $\bar{\psi}$ is $(m, p)$-quasi u.s.c. since each function $\psi_{h}$ is $(m, p)$-quasi continuous. We now prove that $K_{0}=K(\bar{\psi})$. 
Let us show that $K_{0} \subseteq K(\bar{\psi})$. Let $u \in K_{0}$. There exists a subsequence $\left(v_{h_{k}}\right)$ of $\left(v_{h}\right)$ which converges to $u$ in $W^{m, p}$; by proposition 1.3 we may suppose that $\left(\tilde{v}_{h_{k}}\right)$ converges to $\tilde{u}(m, p)$-q.e. Since for every $k \in \mathbb{N}$, $\psi_{h_{k}} \leq \tilde{v}_{h_{k}}(m, p)$-q.e., we get $\bar{\psi} \leq \tilde{u}(m, p)$-q.e., i.e. $u \in K(\bar{\psi})$. Therefore $K_{0} \subseteq K(\bar{\psi})$.

Let us prove now that $K(\bar{\psi}) \subseteq K_{0}$. Consider $u \in K(\bar{\psi})$ and for every $h \in \mathbb{N}$ let $f_{h}=\psi_{h} \vee \tilde{u}$; then $\left(f_{h}\right)$ decreases to $\tilde{u}(m, p)$-q.e. By lemma 2.3 we can find a sequence $\left(u_{h}\right)$ in $W^{m, p}$ which converges to $u$ in $W^{m, p}$ and such that for every $h \in \mathbb{N}, \tilde{u}_{h} \geq f_{h}(m, p)$-q.e. By $(\beta)_{h}, u_{h} \in K_{0}$ for every $h \in \mathbb{N}$, hence $u \in K_{0}$ since $K_{0}$ is closed in $W^{m, p}$. We conclude that $K(\bar{\psi}) \subseteq K_{0}$.

If $\overline{\psi_{1}}$ and $\overline{\psi_{2}}$ are two $(m, p)$-quasi u.s.c. functions such that $K\left(\overline{\psi_{1}}\right)=$ $K\left(\overline{\psi_{2}}\right)=K_{0}$, we get easily that $\overline{\psi_{1}}=\overline{\psi_{2}}(m, p)$-q.e. by approximating both of them $(m, p)$-q.e. by means of lemma 2.1 .

(ii) Consider the general case of $\Omega$ open subset of $\mathbb{R}^{n}$. By part (i) of the proof and by proposition 2.8 there exists an $(m, p)$-quasi u.s.c. function $\bar{\psi}: \mathbb{R}^{n} \rightarrow \mathbb{R}$ such that $\mathcal{K}\left(K_{0}\right)=K(\bar{\psi})$; moreover $\bar{\psi} \leq 0(m, p)$-q.e. on $\mathbb{R}^{n} \backslash \Omega$. Now from the equality $K_{0}=\mathcal{K} \uparrow \Omega\left(K_{0}\right) \cap W_{0}^{m, p}(\Omega)$, stated in lemma 2.7, we obtain that $K_{0}=K_{0}\left(\bar{\psi} \Gamma_{\Omega}\right)$ with $\bar{\psi} \Gamma_{\Omega}(m, p)$-quasi u.s.c. Finally, the uniqueness of such an obstacle function follows from the corresponding property for $\mathcal{K}\left(K_{0}\right)$.

Proposition 2.10. - Let $\psi: \Omega \rightarrow \overline{\mathbb{R}}$ be a function such that $K_{0}(\psi) \neq \emptyset$. If $\bar{\psi}: \Omega \rightarrow \mathbb{R}$ is the $(m, p)$-quasi u.s.c. function, given in proposition 2.9, for which $K_{0}(\psi)=K_{0}(\bar{\psi})$, then $\bar{\psi}$ is the $(m, p)$-quasi u.s.c. envelope of $\psi$ (i.e. the least $(m, p)$-quasi u.s.c. function $f: \Omega \rightarrow \overline{\mathbb{R}}$ with $f \geq \psi(m, p)$-q.e. on $\Omega$ ).

Proof. - Assume at first $\Omega=\mathbb{R}^{n}$. By lemma 2.1 we easily obtain that $\bar{\psi} \geq \psi(m, p)$-q.e. Fix now an $(m, p)$-quasi u.s.c. function $f: \Omega \rightarrow \mathbb{R}$ with $f \geq \psi(m, p)$-q.e. By lemma 2.1 we can find a sequence $\left(u_{h}\right)$ in $W^{m, p}$ such that $\left(\tilde{u}_{h}\right)$ decreases and converges to $f \wedge \bar{\psi}(m, p)$-q.e. Since $f \wedge \bar{\psi} \geq \psi$ $(m, p)$-q.e., we have $u_{h} \in K_{0}(\psi)$ for every $h \in \mathbb{N}$, then $\tilde{u}_{h} \geq \bar{\psi}$ : taking the limit $(m, p)$-q.e. we have $f \wedge \bar{\psi} \geq \bar{\psi}$, i.e. $f \geq \bar{\psi}(m, p)$-q.e. We conclude that $\bar{\psi}$ is the $(m, p)$-quasi u.s.c. envelope of $\psi$.

Consider the general case of $\Omega$ open subset of $\mathbb{R}^{n}$. Let $\overline{T_{\Omega} \psi}$ denote the $(m, p)$-quasi u.s.c. envelope of $T_{\Omega} \psi$; then $\overline{T_{\Omega} \psi} \leq 0(m, p)$-q.e. on $\mathbb{R}^{n} \backslash \Omega$. 
By lemma 2.7 and what just proved we have

$$
\begin{aligned}
K_{0}(\psi) & =\left\{u \uparrow_{\Omega} \mid u \in K\left(T_{\Omega} \psi\right)\right\} \cap W_{0}^{m, p}(\Omega) \\
& =\left\{u \uparrow_{\Omega} \mid u \in K\left(\overline{T_{\Omega} \psi}\right)\right\} \cap W_{0}^{m, p}(\Omega)=K_{0}\left(\overline{T_{\Omega} \psi}\right) .
\end{aligned}
$$

Moreover it is easy to check that $\left(\overline{T_{\Omega} \psi}\right) \Omega_{\Omega}$ is the $(m, p)$-quasi u.s.c. envelope of $\psi$.

Remark 2.11. - Consider a subset $E$ of $\Omega$ and its quasi closure $\widetilde{E}$. If $K_{0}\left(\mathbf{1}_{E}\right) \neq \emptyset$, then $\widetilde{E}$ is quasi contained in $\Omega$ and $K_{0}\left(\mathbf{1}_{E}\right)=K_{0}\left(\mathbf{1}_{\tilde{E}}\right)$ (indeed, if $u \in K_{0}\left(\mathbf{1}_{E}\right)$, then $\{x \in \Omega \mid \tilde{u}(x) \geq 1\}$ is quasi closed and quasi contains $E)$. Hence, in particular, the capacities $C_{m, p}$ and $B_{m, p}$ we are dealing with are "quasi stable" in the sense that every set has the same capacity as its quasi closure (see [17]).

It is now immediate to prove the closure, with respect to the Mosco convergence, of the class of closed unilateral convex sets in $W_{0}^{m, p}(\Omega)$.

Proposition 2.12. - If $\left(\psi_{h}\right)$ is a sequence of functions from $\Omega$ into $\overline{\mathbb{R}}$, then the strong lower limit $K_{0}^{\prime}\left(\left(\psi_{h}\right)\right)$ of the sequence $\left(K_{0}\left(\psi_{h}\right)\right)$ is a closed unilateral convex set in $W_{0}^{m, p}(\Omega)$.

Proof. - We have already remarked that $K_{0}^{\prime}\left(\left(\psi_{h}\right)\right)$ is strongly closed in $W_{0}^{m, p}(\Omega)$. Let us show that conditions $(\alpha)$ holds (stability with respect to addition of non-negative $W_{0}^{m, p}(\Omega)$ functions).

Fix $u \in K_{0}^{\prime}\left(\left(\psi_{h}\right)\right)$ and $v \in W_{0}^{m, p}(\Omega)$ with $v \geq u$ a.e. on $\Omega$; we can find a sequence $\left(u_{h}\right)$ converging to $u$ in $W_{0}^{m, p}(\Omega)$ with $u_{h} \in K_{0}\left(\psi_{h}\right)$ whenever $h \geq k$, for a suitable index $k \in \mathbb{N}$. If we put $v_{h}=u_{h}+(v-u)$ for every $h \in \mathbb{N}$, then we obtain a sequence $\left(v_{h}\right)$ converging to $v$ in $W_{0}^{m, p}(\Omega)$ and such that $v_{h} \in K_{0}\left(\psi_{h}\right)$ for $h \geq k$. Thus $v \in K_{0}^{\prime}\left(\left(\psi_{h}\right)\right)$.

Since we have just proved condition $(\alpha)$, and $K_{0}^{\prime}\left(\left(\psi_{h}\right)\right)$ is trivially $C^{\infty}$-convex, we conclude by proposition 2.8 that $K_{0}^{\prime}\left(\left(\psi_{h}\right)\right)$ is a unilateral convex set.

We state now two useful consequences of proposition 2.12.

CoRollary 2.13. - Let $\left(\psi_{h}\right)$ be a sequence of functions from $\Omega$ into $\overline{\mathbb{R}}$ such that $K_{0}^{\prime}\left(\left(\psi_{h}\right)\right) \neq \emptyset$. Then

$$
K_{0}^{\prime}\left(\left(\psi_{h}\right)\right)=\bigcap_{\omega \Subset \Omega} K_{0}^{\prime}\left(\left(\psi_{h}\right), \omega\right),
$$

where the intersection runs over all the open sets $\omega \Subset \Omega$. 
Proof. - Only one inclusion is not trivial.

Let us fix $u \in \bigcap_{\omega \Subset \Omega} K_{0}^{\prime}\left(\left(\psi_{h}\right), \omega\right)$ and show that $u \in K_{0}^{\prime}\left(\left(\psi_{h}\right)\right)$. As $K_{0}^{\prime}\left(\left(\psi_{h}\right)\right) \neq \emptyset$ it easy to see that there is no loss of generality if we assume $\psi_{h} \leq 0(m, p)$-q.e. on $\Omega$ for every $h \in \mathbb{N}$. Consider open sets $\omega$ and $\omega^{\prime}$ with $\omega \Subset \omega^{\prime} \Subset \Omega$ and a function $\varphi \in C_{0}^{\infty}\left(\omega^{\prime}\right)$ with $\varphi=1$ on $\omega$ and $0 \leq \varphi \leq 1$. Since $u \in K_{0}^{\prime}\left(\left(\psi_{h}\right), \omega^{\prime}\right)$ we get $\varphi u \in K_{0}^{\prime}\left(\left(\psi_{h}\right)\right)$. By propositions 2.12 and 2.9 there exists a function $\psi: \Omega \rightarrow \overline{\mathbb{R}}$ such that $K_{0}^{\prime}\left(\left(\psi_{h}\right)\right)=K_{0}(\psi)$. Therefore $\tilde{u}=\varphi \tilde{u} \geq \psi(m, p)$-q.e. on $\omega$. Since $\omega$ is arbitrary we conclude that $u \in K_{0}(\psi)=K_{0}^{\prime}\left(\left(\psi_{h}\right)\right)$.

Proposition 2.14. - Let $f$ and $\left(f_{h}\right)$ be a function and a sequence of functions, respectively, from $\Omega$ into $\mathbb{R}$. Let $u \in W_{0}^{m, p}(\Omega)$. Assume one of the following hypotheses:

(i) $f_{h}$ is $(m, p)$-quasi u.s.c. for every $h \in \mathbb{N},\left(f_{h}\right)$ is decreasing and converges to $\tilde{u}(m, p)$-q.e. on $\Omega$, and there exists a function $w \in W_{0}^{m, p}(\Omega)$ such that $\tilde{w} \geq f_{1}(m, p)$-q.e. on $\Omega$;

(ii) $\left(f_{h}\right)$ converges to $\tilde{u}$ in $(m, p)$-capacity and there exists a sequence $\left(w_{h}\right)$, which converges strongly in $W_{0}^{m, p}(\Omega)$, such that $f_{h} \leq \tilde{w}_{h}$ $(m, p)$-q.e. on $\Omega$ for every $h \in \mathbb{N}$.

Then we can find a sequence $\left(u_{h}\right)$ in $W_{0}^{m, p}(\Omega)$, which converges to $u$ strongly in $W_{0}^{m, p}(\Omega)$, and for which $\tilde{u}_{h} \geq f_{h}(m, p)$-q.e. on $\Omega$ for every $h \in \mathbb{N}$.

Proof. - Assume (i) (case (ii) can be handled in a similar way); we may suppose that $f_{h} \leq 0(m, p)$-q.e. on $\Omega$ for every $h \in \mathbb{N}$. Let us consider the functions $f_{h}$ and $u$ as defined all over $\mathbb{R}^{n}$ with value zero on $\mathbb{R}^{n} \backslash \Omega$ : $\left(f_{h}\right)$ is a sequence of $(m, p)$-quasi u.s.c. functions on $\mathbb{R}^{n}$ and $u \in W^{m, p}$. Then lemma 2.3 yields that $u \in K^{\prime}\left(\left(f_{h}\right)\right)$, thus $u \in \mathcal{K}\left(K_{0}^{\prime}\left(\left(f_{h}\right)\right)\right)$, as one can easily check. By propositions 2.12 and $2.9, K_{0}^{\prime}\left(\left(f_{h}\right)\right)$ is a unilateral convex set of obstacle type; on account of the definition of $\mathcal{K}\left(K_{0}^{\prime}\left(\left(f_{h}\right)\right)\right)$ we conclude that $u \in K_{0}^{\prime}\left(\left(f_{h}\right)\right)$. This is what we had to prove.

We conclude this section with a few remarks about the connection between convex sets of obstacle type in the spaces $W^{m, p}$ and $L^{p}\left(B_{m, p}\right)$. The spaces $L^{p}\left(B_{m, p}\right)$ have a kind of convergence naturally modelled on $W^{m, p}$, but their elements are not required to possess derivatives: so they may be more suitable when truncation arguments are involved. $L^{p}$-spaces with respect to a capacity were introduced in [2] for the Riesz capacity.

We recall now the definitions and some properties we shall need (see [5]). 
DEFINITION 2.15. - Let $Q_{m, p}$ denote the space of $(m, p)$-quasi continuous functions from $\mathbb{R}^{n}$ into $\mathbb{R}$ (with the equivalence relation of $(m, p)$-q.e. equality). Define

$$
L^{p}\left(B_{m, p}\right)=\left\{f \in Q_{m, p}\left|\exists u \in W^{m, p},\right| f \mid \leq \tilde{u}(m, p)-q . e .\right\},
$$

and for every $f \in L^{p}\left(B_{m, p}\right)$

$$
\begin{aligned}
\|f\|_{L^{p}\left(B_{m, p}\right)} & =\left(B_{m, p}(|f|)\right)^{1 / p} \\
& =\inf \left\{\|u\|_{m, p}\left|u \in W^{m, p},\right| f \mid \leq \tilde{u}(m, p) \text {-q.e. }\right\} .
\end{aligned}
$$

Proposition 2.16

(i) $\|\cdot\|_{L^{p}\left(B_{m, p}\right)}$ is an increasing norm on $L^{p}\left(B_{m, p}\right)$ with respect to which $L^{p}\left(B_{m, p}\right)$ is a Banach space;

(ii) $W^{m, p}$ is continuously imbedded in $L^{p}\left(B_{m, p}\right)$.

The proof can be easily obtained as in proposition 1.2 of [5] taking into account theorem 2.2 .

Remark 2.17. - Let $\left(f_{h}\right)$ be a sequence of functions in $L^{p}\left(B_{m, p}\right)$ which is decreasing and converges $(m, p)$-q.e. to a function $f \in L^{p}\left(B_{m, p}\right)$. Then it immediately follows from lemma 2.3 that $\left(f_{h}\right)$ converges to $f$ in $L^{p}\left(B_{m, p}\right)$. By proposition 1.3 we also have that convergence in $L^{p}\left(B_{m, p}\right)$ implies convergence in $(m, p)$-capacity and, up to a subsequence, convergence $(m, p)$-q.e.

Moreover, we observe that if $f \in L^{p}\left(B_{m, p}\right)$ and $g$ is either an $(m, p)$-quasi continuous non-negative function or $g \in L^{p}\left(B_{m, p}\right)$ then $f \wedge g \in L^{p}\left(B_{m, p}\right)$; furthermore the mapping $f \mapsto f \wedge g$ is continuous from $L^{p}\left(B_{m, p}\right)$ into $L^{p}\left(B_{m, p}\right)$. A similar statement holds for $\vee$.

For every $\psi: \mathbb{R}^{n} \rightarrow \overline{\mathbb{R}}$ define

$$
\tilde{K}(\psi)=\left\{f \in L^{p}\left(B_{m, p}\right) \mid f \geq \psi(m, p) \text { - q.e. }\right\} .
$$

We remark that $\tilde{K}(\psi)$ contains $K(\psi)$ (provided that the functions of $K(\psi)$ are identified with their $(m, p)$-quasi continuous representatives). Moreover, $\tilde{K}(\psi)$ is closed in $L^{p}\left(B_{m, p}\right)$; more precisely:

Proposition 2.18. $-\tilde{K}(\psi)$ is the closure of $K(\psi)$ in $L^{p}\left(B_{m, p}\right)$. 
Proof. - Let $f \in \tilde{K}(\psi)$; by lemma 2.3 there exists a sequence $\left(u_{h}\right)$ in $W^{m, p}$ such that $\left(\tilde{u}_{h}\right)$ decreases and converges to $f(m, p)$-q.e. Then $u_{h} \in K(\psi)$ for every $h \in \mathbb{N}$ and, on account of remark $2.17,\left(\tilde{u}_{h}\right)$ converges to $f$ in $L^{p}\left(B_{m, p}\right)$. Therefore $f$ is in the closure of $K(\psi)$ in $L^{p}\left(B_{m, p}\right)$.

Given a sequence of functions $\left(\psi_{h}\right)$ from $\mathbb{R}^{n}$ into $\overline{\mathbb{R}}$, we set

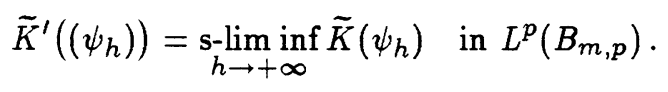

Proposition 2.19. $-K(\psi) \subseteq K^{\prime}\left(\left(\psi_{h}\right)\right)$ if and only if $\tilde{K}(\psi) \subseteq$ $\tilde{K}^{\prime}\left(\left(\psi_{h}\right)\right)$.

Proof. - Assume that $K(\psi) \subseteq K^{\prime}\left(\left(\psi_{h}\right)\right)$. By proposition 2.16, $K^{\prime}\left(\left(\psi_{h}\right)\right) \subseteq \tilde{K}^{\prime}\left(\left(\psi_{h}\right)\right)$ so that proposition 2.18 yields

$$
\tilde{K}(\psi) \subseteq \operatorname{cl}_{L^{p}\left(B_{m, p}\right)}\left(K^{\prime}\left(\left(\psi_{h}\right)\right)\right) \subseteq \tilde{K}^{\prime}\left(\left(\psi_{h}\right)\right),
$$

since $\tilde{K}^{\prime}\left(\left(\psi_{h}\right)\right)$ is closed in $L^{p}\left(B_{m, p}\right)$. Therefore $\tilde{K}(\psi) \subseteq \tilde{K}^{\prime}\left(\left(\psi_{h}\right)\right)$.

Assume now that $\tilde{K}(\psi) \subseteq \tilde{K}^{\prime}\left(\left(\psi_{h}\right)\right)$. Let $u \in K(\psi)$. Since $K(\psi) \subseteq$ $\tilde{K}(\psi)$, we can find an index $k \in \mathbb{N}$ and a sequence $\left(f_{h}\right)$ in $L^{p}\left(B_{m, p}\right)$ converging to $\tilde{u}$ in $L^{p}\left(B_{m, p}\right)$ such that $f_{h} \in \tilde{K}\left(\psi_{h}\right)$ for every $h \geq k$. By remark 2.17 and lemma 2.3 there exists a sequence $\left(u_{h}\right)$ in $W^{m, p}$ which converges to $u$ in $W^{m, p}$ and for which $\tilde{u}_{h} \geq f_{h}(m, p)$-q.e. We conclude that $u \in K^{\prime}\left(\left(\psi_{h}\right)\right)$, therefore $K(\psi) \subseteq K^{\prime}\left(\left(\psi_{h}\right)\right)$.

3. Convergence of obstacles and level sets: the case of equi-bounded obstacles on a compact set

Following the study carried out in [9] for the case $m=1$, we now characterize the Mosco convergence of the sequence $\left(K_{0}\left(\psi_{h}\right)\right)$ to the set $K_{0}(\psi)$ through the Mosco convergence of $\left(K_{0}\left(\mathbf{1}_{\left\{\psi_{h}>t\right\}}\right)\right)$ to $K_{0}\left(\mathbf{1}_{\{\psi>t\}}\right)$, for $t$ in a dense subset of $\mathbb{R}$ (if $f: \Omega \rightarrow \mathbb{R}$ is any function and $t \in \mathbb{R}$, by $\{f>t\}$ we denote the level set $\{x \in \Omega \mid f(x)>t\}$ ). The main result in this section is theorem 3.1, where the obstacles are assumed to be equi-bounded from above and equal to $-\infty$ outside a fixed compact subset of $\Omega$. These additional conditions will be considerably weakened in the next section. 
TheOREM 3.1. - Let $\left(\psi_{h}\right)$ be a sequence of functions from $\Omega$ into $\overline{\mathbb{R}}$ and let $\psi$ be a function from $\Omega$ into $\overline{\mathbb{R}}$. Assume that there exist a set $\Omega^{\prime} \Subset \Omega$ and a constant $M>0$ such that for every $h \in \mathbb{N}$ :

$$
\psi, \psi_{h}=-\infty \quad \text { on } \Omega \backslash \Omega^{\prime} \quad ; \quad \psi, \psi_{h} \leq M \quad \text { on } \Omega^{\prime} .
$$

Then

$$
K_{0}\left(\psi_{h}\right) \rightarrow K_{0}(\psi) \quad \text { in } W_{0}^{m, p}(\Omega)
$$

if and only if there exists a dense set $D$ in $\mathbb{R}$ such that for every $t \in D$

$$
K_{0}\left(\mathbf{1}_{\left\{\psi_{h}>t\right\}}\right) \rightarrow K_{0}\left(\mathbf{1}_{\{\psi>t\}}\right) \text { in } W_{0}^{m, p}(\Omega) .
$$

Moreover, condition (3.1) is equivalent to

$$
K_{0}\left(\chi_{\left\{\psi_{h}>t\right\}}\right) \rightarrow K_{0}\left(\chi_{\{\psi>t\}}\right) \text { in } W_{0}^{m, p}(\Omega) .
$$

Finally, $D$ can be chosen so that $\mathbb{R} \backslash D$ is countable.

Throughout the following preliminary lemmas, $\psi$ and $\left(\psi_{h}\right)$ are a function and a sequence of functions, respectively, from $\mathbb{R}^{n}$ into $\bar{R}$.

Lemma 3.2. - Let $\Omega^{\prime} \Subset \Omega$. If $K_{0}(\psi)$ and $K(\psi)$ are non-empty, and $\psi_{h}=\psi(m, p)$-q.e. on $\mathbb{R}^{n} \backslash \Omega^{\prime}$ for every $h \in \mathbb{N}$, then the following conditions are equivalent:

(a) $K_{0}\left(\psi_{h}\right) \rightarrow K_{0}(\psi) \quad$ in $W_{0}^{m, p}(\Omega)$;

(b) $K\left(\psi_{h}\right) \rightarrow K(\psi)$ in $W^{m, p}$.

Proof. - Let $\varphi \in C_{0}^{\infty}(\Omega)$ such that $\varphi=1$ on a neighborhood $\Omega^{\prime \prime}$ of $\bar{\Omega}^{\prime}$ and $0 \leq \varphi \leq 1$.

(a) $\Rightarrow$ (b) Assume (a). Since $K_{0}(\psi) \neq \emptyset$ we may suppose that $\psi, \psi_{h} \leq 0$ $(m, p)$-q.e. on $\Omega$ for every $h \in \mathbb{N}$. Let us prove that $K(\psi) \subseteq K^{\prime}\left(\left(\psi_{h}\right)\right)$. Take $u \in K(\psi)$. As $\varphi u \in K_{0}(\psi)$, there exist an index $k \in \mathbb{N}$ and a sequence $\left(v_{h}\right)$ converging to $\varphi u$ in $W_{0}^{m, p}(\Omega)$ such that $v_{h} \in K_{0}\left(\psi_{h}\right)$ if $h \geq k$. Let $\eta \in C_{0}^{\infty}\left(\Omega^{\prime \prime}\right)$ with $\eta=1$ on $\Omega^{\prime}$ and $0 \leq \eta \leq 1$; then $\eta v_{h}+(1-\eta) u \in K\left(\psi_{h}\right)$ for every $h \geq k$, as $\psi_{h}=\psi$ on $\mathbb{R}^{n} \backslash \Omega^{\prime}$, and $\left(\eta v_{h}+(1-\eta) u\right)$ converges to $u$ in $W^{m, p}$. We conclude that $u \in K^{\prime}\left(\left(\psi_{h}\right)\right)$, hence $K(\psi) \subseteq K^{\prime}\left(\left(\psi_{h}\right)\right)$. Moreover, it is simple to see that if $u \in K^{\prime \prime}\left(\left(\psi_{h}\right)\right)$ then $\varphi u \in K_{0}(\psi)$ and $\tilde{u} \geq \psi(m, p)$-q.e. on $\mathbb{R}^{n} \backslash \Omega^{\prime}$. This means that $u \in K(\psi)$, therefore $K^{\prime \prime}\left(\left(\psi_{h}\right)\right) \subseteq K(\psi)$.

(b) $\Rightarrow$ (a) It can be proved in a similar (and even simpler) way. 
Lemma 3.3. - Let $\Omega^{\prime}$ be a subset of $\Omega$ with $\Omega^{\prime} \Subset \Omega$. Assume that $\left(K_{0}\left(\psi_{h}, \Omega^{\prime}\right)\right)$ converges to $K_{0}\left(\psi, \Omega^{\prime}\right)$ in the sense of Mosco in $W_{0}^{m, p}(\Omega)$. Then for every $t \in \mathbb{R}$

$$
K_{0}\left(\psi_{h}+t, \Omega^{\prime}\right) \rightarrow K_{0}\left(\psi+t, \Omega^{\prime}\right) \text { in } W_{0}^{m, p}(\Omega) .
$$

Proof. - Consider a function $\varphi \in C_{0}^{\infty}(\Omega)$ with $\varphi=1$ on $\Omega^{\prime}$; then it suffices to observe that $K_{0}\left(\psi_{h}+t, \Omega^{\prime}\right)=K_{0}\left(\psi_{h}, \Omega^{\prime}\right)+t \varphi$ and $K_{0}\left(\psi+t, \Omega^{\prime}\right)=$ $K_{0}\left(\psi, \Omega^{\prime}\right)+t \varphi$.

LEMMA 3.4. - Let $\left(f_{h}\right)$ be a sequence of functions in $L^{p}$ which converges weakly in $L^{p}$ to a function $f$. Let $\left(t_{i}\right)$ be a sequence of negative real numbers converging to $-\infty$ and assume that to every $i \in \mathbb{N}$ there corresponds a function $g_{i} \in L^{p}$ such that

$$
f_{k} \vee t_{i} \rightarrow g_{i} \quad \text { weakly in } L^{p} \text { as } k \rightarrow+\infty .
$$

Then

$$
g_{i} \rightarrow f \quad \text { weakly in } L^{p} \text { as } i \rightarrow+\infty \text {. }
$$

Proof. - Fix a non-negative function $v \in L^{p^{\prime}}$, where $1 / p+1 / p^{\prime}=1$. Let $M$ be an upper bound of the sequence $\left(\left\|f_{k}\right\|_{L^{p}}\right)$; for every $i, k \in \mathbb{N}$ we have

$$
\begin{aligned}
0 & \leq \int_{\mathbb{R}^{n}}\left(f_{k} \vee t_{i}-f_{k}\right) v \mathrm{~d} x=\int_{\left\{f_{k}<t_{i}\right\}}\left(t_{i}-f_{k}\right) v \mathrm{~d} x \leq \\
& \leq-\int_{\left\{f_{k}<t_{i}\right\}} f_{k} v \mathrm{~d} x \leq M\|v\|_{L^{p^{\prime}}\left(\left\{f_{k}<t_{i}\right\}\right)} .
\end{aligned}
$$

Moreover, by Chebyshev's inequality $\left|\left\{f_{k}<t_{i}\right\}\right|$ tends to zero as $i \rightarrow+\infty$ uniformly with respect to $k$. Thus

$$
\lim _{i \rightarrow+\infty} \int_{\mathbb{R}^{n}}\left(f_{k} \vee t_{i}-f_{k}\right) v \mathrm{~d} x=0
$$

uniformly with respect to $k$, and then

$$
\lim _{i \rightarrow+\infty} \int_{\mathbb{R}^{n}}\left(g_{i}-f\right) v \mathrm{~d} x=0 .
$$

For a general $v \in L^{p^{\prime}}$ it suffices to consider its positive and negative parts. 
LEMмA 3.5. - Let $T$ be an unbounded set of non-positive real numbers. Then the following conditions are equivalent:

(a) $K\left(\psi_{h} \vee t\right) \rightarrow K(\psi \vee t) \quad$ in $W^{m, p}$ for every $t \in T$;

(b) $K\left(\psi_{h}\right) \rightarrow K(\psi)$ in $W^{m, p}$.

\section{Proof}

(a) $\Rightarrow$ (b) Let us show first that $K(\psi) \subseteq K^{\prime}\left(\left(\psi_{h}\right)\right)$. Let $u$ be in $K(\psi)$ and $\left(t_{i}\right)$ a sequence in $T$ converging to $-\infty$. If $u=J_{m} f$ with $f \in L^{p}$, put $v_{i}=G_{m} \star\left(f \vee t_{i}\right)$ for every $i \in \mathbb{N}$. By assumption (a), $v_{i} \in K\left(\psi \vee t_{i}\right) \subseteq K^{\prime}\left(\left(\psi_{h} \vee t_{i}\right)_{h}\right)$, then for every $i \in \mathbb{N}, v_{i}$ is in $K^{\prime}\left(\left(\psi_{h}\right)\right)$. This latter is closed in $W^{m, p}$ and $\left(v_{i}\right)$ converges to $u$ strongly in $W^{m, p}$, hence $u \in K^{\prime}\left(\left(\psi_{h}\right)\right)$. Therefore $K(\psi) \subseteq K^{\prime}\left(\left(\psi_{h}\right)\right)$.

Now we prove that $K^{\prime \prime}\left(\left(\psi_{h}\right)\right) \subseteq K(\psi)$. Let $\left(h_{k}\right)$ be a strictly increasing sequence of positive integers and let $u$ be the weak limit in $W^{m, p}$ of a sequence $\left(u_{k}\right)$ such that $u_{k} \in K\left(\psi_{h_{k}}\right)$ for every $k \in \mathbb{N}$. If $f=\left(J_{m}\right)^{-1} u$ and $f_{k}=\left(J_{m}\right)^{-1} u_{k}(k \in \mathbb{N})$, then $\left(f_{k}\right)$ converges to $f$ weakly in $L^{p}$. Let $\left(t_{i}\right)$ be a sequence in $T$ which converges to $-\infty$; by means of a diagonal argument we may suppose that for every $i \in \mathbb{N}$ there exists a function $g_{i} \in L^{p}$ such that $f_{k} \vee t_{i}$ converges to $g_{i}$ weakly in $L^{p}$ as $k \rightarrow+\infty$. Since $G_{m} \star\left(f_{k} \vee t_{i}\right) \in K\left(\psi_{h_{k}} \vee t_{i}\right)$ and $\left(G_{m} \star\left(f_{k} \vee t_{i}\right)\right)$ converges to $G_{m} \star g_{i}$ weakly in $W^{m, p}$ as $k \rightarrow+\infty$, by assumption (a) $G_{m} \star g_{i} \in K\left(\psi \vee t_{i}\right) \subseteq K(\psi)$ for every $i \in \mathbb{N}$. By the previous lemma, $\left(G_{m} \star g_{i}\right)$ converges weakly in $W^{m, p}$ to $G_{m} \star f=u$, so that $u \in K(\psi)$ as $K(\psi)$ is convex and strongly closed in $W^{m, p}$. We conclude that $K^{\prime \prime}\left(\left(\psi_{h}\right)\right) \subseteq K(\psi)$.

(b) $\Rightarrow$ (a) Fix $t \in T$. We show that $K(\psi \vee t) \subseteq K^{\prime}\left(\left(\psi_{h} \vee t\right)\right)$. Let $u \in K(\psi \vee t)$ and $u=J_{m} f$, with $f \in L^{p}$; in particular $u \in K(\psi)$ thus, by assumption, we can find an index $k \in \mathbb{N}$ and a sequence $\left(v_{h}\right)$ which converges to $u$ in $W^{m, p}$ and such that $v_{h} \in K\left(\psi_{h}\right)$ for every $h \geq k$. If $f_{h}=\left(J_{m}\right)^{-1} v_{h}$, put $z_{h}=G_{m} \star\left(f_{h} \vee f\right)$ for every $h \in \mathbb{N}$; when $h \geq k$ we have

$$
z_{h}=G_{m} \star\left(f_{h} \vee f\right) \geq \tilde{v}_{h} \vee \tilde{u} \geq \psi_{h} \vee t \quad(m, p) \text {-q.e. ; }
$$

therefore $z_{h} \in K\left(\psi_{h} \vee t\right)$. Moreover, $\left(z_{h}\right)$ converges to $u$ in $W^{m, p}$, as $\left(f_{h}\right)$ converges to $f$ in $L^{p}$, so that $u \in K^{\prime}\left(\left(\psi_{h} \vee t\right)\right)$. Therefore $K(\psi \vee t) \subseteq$ $K^{\prime}\left(\left(\psi_{h} \vee t\right)\right)$.

Finally, if $u$ is a function in $K^{\prime \prime}\left(\left(\psi_{h} \vee t\right)\right)$, then $\tilde{u} \geq t(m, p)$-q.e. and $u \in K^{\prime \prime}\left(\left(\psi_{h}\right)\right)$; by condition (b), $u \in K(\psi \vee t)$. We have thus proved that $K^{\prime \prime}\left(\left(\psi_{h} \vee t\right)\right) \subseteq K(\psi \vee t)$. 
CoRollary 3.6. - Let $\left(E_{h}\right)$ be a sequence of subsets of $\mathbb{R}^{n}$ and let $E$ be a subset of $\mathbb{R}^{n}$. If there exists a set $\Omega^{\prime} \Subset \Omega$ which contains $E$ and the sequence $\left(E_{h}\right)$, then the following conditions are equivalent:

(a) $K_{0}\left(\mathbf{1}_{E_{h}}\right) \rightarrow K_{0}\left(\mathbf{1}_{E}\right)$ in $W_{0}^{m, p}(\Omega)$;

(b) $K_{0}\left(\chi_{E_{h}}\right) \rightarrow K_{0}\left(\chi_{E}\right)$ in $W_{0}^{m, p}(\Omega)$.

Proof. - By lemma 3.2 it suffices to consider the case $\Omega=\mathbb{R}^{n}$.

(b) $\Rightarrow$ (a) It immediately follows from lemma 3.3 and part (b) $\Rightarrow$ (a) of lemma 3.5.

(a) $\Rightarrow$ (b) Consider a bounded open set $\Omega^{\prime \prime} \ni \Omega^{\prime}$; it is easy to check (see the proof of lemma 3.2) that condition (a) implies the convergence of $\left(K\left(\mathbf{1}_{E_{h}}, \Omega^{\prime \prime}\right)\right)$ to $K\left(1_{E}, \Omega^{\prime \prime}\right)$. On account of the boundedness of $\Omega^{\prime \prime}$, by lemma $3.3\left(K\left(\mathbf{1}_{E_{h}}-1, \Omega^{\prime \prime}\right)\right)$ converges to $K\left(\mathbf{1}_{E}-1, \Omega^{\prime \prime}\right)$ and then for every real $t<0,\left(K\left(\chi_{E_{h}} \vee t, \Omega^{\prime \prime}\right)\right)$ converges to $K\left(\chi_{E} \vee t, \Omega^{\prime \prime}\right)$. Part (b) $\Rightarrow$ (a) of the previous lemma now gives that

$$
K\left(\chi_{E_{h}} \vee t\right) \rightarrow K\left(\chi_{E} \vee t\right) \quad \text { for every } t<0 .
$$

By part $(\mathrm{a}) \Rightarrow(\mathrm{b})$ of the same lemma, we conclude that $\left(K\left(\chi_{E_{h}}\right)\right)$ converges to $K\left(\chi_{E}\right)$.

LEMmA 3.7. - Assume that $K(\psi) \neq \emptyset$; let $\bar{\psi}$ be the $(m, p)$-quasi u.s.c. envelope of $\psi$ (see proposition 2.10). If $t \in \mathbb{R}^{+}$, then $\bar{\psi} \wedge t$ and $(\bar{\psi}-t)^{+}$are the $(m, p)$-quasi u.s.c. envelopes of $\psi \wedge t$ and $(\psi-t)^{+}$, respectively.

Proof. $-\bar{\psi} \wedge t$ is quasi u.s.c. and majorizes $\psi \wedge t$. On the other hand, let $f: \mathbb{R}^{n} \rightarrow \mathbb{\mathbb { R }}$ be a quasi u.s.c. function with $f \geq \psi \wedge t$ ( $m, p)$-q.e.; since $f+(\bar{\psi}-t)^{+} \geq \psi \wedge t+(\psi-t)^{+}=\psi(m, p)$-q.e., then $f+(\bar{\psi}-t)^{+} \geq \bar{\psi}(m, p)$-q.e. Moreover $\bar{\psi}<+\infty(m, p)$-q.e. as $K(\bar{\psi}) \neq \emptyset$; hence $f \geq \bar{\psi}-(\bar{\psi}-t)^{+}=\bar{\psi} \wedge t(m, p)$-q.e. We conclude that $\bar{\psi} \wedge t$ is the quasi u.s.c. envelope of $\psi \wedge t$. The case $(\psi-t)^{+}$can be handled in a similar way.

LEMMA 3.8. - Let $t \in \mathbb{R}^{+}$. Assume that

$$
\begin{aligned}
K(\psi \wedge t) & \subseteq K^{\prime}\left(\left(\psi_{h} \wedge t\right)\right) \\
K\left((\psi-t)^{+}\right) & \subseteq K^{\prime}\left[\left(\left(\psi_{h}-t\right)^{+}\right)\right] .
\end{aligned}
$$

Then $K(\psi) \subseteq K^{\prime}\left(\left(\psi_{h}\right)\right)$ 
Proof. - By proposition 2.19, lemma 3.8 may be restated in the following form: given $t \in \mathbb{R}^{+}$, if

$$
\begin{aligned}
\tilde{K}(\psi \wedge t) & \subseteq \tilde{K}^{\prime}\left(\left(\psi_{h} \wedge t\right)\right) \\
\tilde{K}\left((\psi-t)^{+}\right) & \subseteq \tilde{K}^{\prime}\left[\left(\left(\psi_{h}-t\right)^{+}\right)\right],
\end{aligned}
$$

then $\tilde{K}(\psi) \subseteq \tilde{K}^{\prime}\left(\left(\psi_{h}\right)\right)$. This follows easily from the identity $f=(f \wedge t)+$ $(f-t)^{+}$.

Lemma 3.9. - Assume that $K(\psi) \neq \emptyset$ and that $\left(K\left(\psi_{h}\right)\right)$ converges to $K(\psi)$ in $W^{m, p}$. Then

$$
\begin{aligned}
K\left(\psi_{h} \wedge t\right) & \rightarrow K(\psi \wedge t) \quad \text { in } W^{m, p} \\
K\left(\left(\psi_{h}-t\right)^{+}\right) & \rightarrow K\left((\psi-t)^{+}\right) \quad \text { in } W^{m, p}
\end{aligned}
$$

for every $t \in \mathbb{R}^{+}$.

Proof. - Fix $t \in \mathbb{R}^{+}$. Let us prove that $K(\psi \wedge t) \subseteq K^{\prime}\left(\left(\psi_{h} \wedge t\right)\right)$ by showing that $\tilde{K}(\psi \wedge t) \subseteq \tilde{K}^{\prime}\left(\left(\psi_{h} \wedge t\right)\right)$ (see proposition 2.19). Let $f \in \tilde{K}(\psi \wedge t)$. By assumption and by proposition $2.19, \tilde{K}(\psi) \subseteq \tilde{K}^{\prime}\left(\left(\psi_{h}\right)\right)$. Consequently, for every function $g \in \tilde{K}(\psi)$ there exists a sequence $\left(g_{h}\right)$ converging to $g$ in $L^{p}\left(B_{m, p}\right)$ such that $g_{h} \in K\left(\psi_{h}\right)$ for $h$ large enough. By remark 2.17, $\left(\left(g_{h} \wedge t\right) \vee f\right)$ converges to $(g \wedge t) \vee f$ in $L^{p}\left(B_{m, p}\right)$ and $\left(g_{h} \wedge t\right) \vee f \in \tilde{K}\left(\psi_{h} \wedge t\right)$ for $h$ large enough. Then $(g \wedge t) \vee f \in \tilde{K}^{\prime}\left(\left(\psi_{h} \wedge t\right)\right)$. Let $\bar{\psi}$ be the quasi u.s.c. envelope of $\psi$ (see proposition 2.10); by lemma 2.1 it is possible to find a sequence $\left(f_{h}\right)$ of quasi continuous functions, which decreases and converges to $\bar{\psi}(m, p)$-q.e. Take the elements of $\left(f_{h}\right)$ as $g$ in the previous result: $\left(\left(f_{h} \wedge t\right) \vee f\right)$ is a sequence in $\widetilde{K}^{\prime}\left(\left(\psi_{h} \wedge t\right)\right)$ which decreases and converges to $f(m, p)$-q.e., as $f \geq \bar{\psi} \wedge t(m, p)$-q.e. by lemma 3.7 ; actually we have convergence in $L^{p}\left(B_{m, p}\right)$, by remark 2.17 . Since $\tilde{K}^{\prime}\left(\left(\psi_{h} \wedge t\right)\right)$ is closed in $L^{p}\left(B_{m, p}\right)$, it must contain $f$, too. Therefore

$$
\tilde{K}(\psi \wedge t) \subseteq \tilde{K}^{\prime}\left(\left(\psi_{h} \wedge t\right)\right)
$$

In a similar way one can obtain the inclusion

$$
K\left((\psi-t)^{+}\right) \subseteq K^{\prime}\left[\left(\left(\psi_{h}-t\right)^{+}\right)\right] .
$$

Let us now prove that $K^{\prime \prime}\left(\left(\psi_{h}-t\right)^{+}\right) \subseteq K\left((\psi-t)^{+}\right)$. Let $u \in$ $K^{\prime \prime}\left(\left(\psi_{h}-t\right)^{+}\right)$. There exist a strictly increasing sequence of positive 
integers $\left(h_{k}\right)$ and a sequence $\left(u_{k}\right)$ converging to $u$ weakly in $W^{m, p}$, such that $u_{k} \in K\left(\left(\psi_{h_{k}}-t\right)^{+}\right)$for every $k \in \mathbb{N}$. The inclusion $K(\psi \wedge t) \subseteq K^{\prime}\left(\left(\psi_{h} \wedge t\right)\right)$ proved above yields that for every $v \in K(\psi \wedge t)$ there exist an index $k_{0}$ and a sequence $\left(v_{h}\right)$ converging to $v$ strongly in $W^{m, p}$, such that $v_{h} \in K\left(\psi_{h} \wedge t\right)$ for every $h \geq k_{0}$. We may assume that $h_{k} \geq k_{0}$ for every $k \in \mathbb{N}$. The sequence $\left(u_{k}+v_{h_{k}}\right)$ converges to $u+v$ weakly in $W^{m, p}$. Moreover, for every $k \in \mathbb{N}$

$$
\tilde{u}_{k}+\tilde{u}_{h_{k}} \geq\left(\psi_{h_{k}} \wedge t\right)+\left(\psi_{h_{k}}-t\right)^{+}=\psi_{h_{k}} \quad(m, p) \text {-q.e. }
$$

thus, by the assumption on $K\left(\psi_{h}\right), u+v \in K(\psi)$, i.e. $u+v \in K(\bar{\psi})$ for every $v \in K(\psi \wedge t)$. By lemma 2.1 , there exists a sequence $\left(w_{h}\right)$ in $K(\bar{\psi} \wedge t)$ such that $\left(\tilde{w}_{h}\right)$ converges to $\bar{\psi} \wedge t(m, p)$-q.e. If we take the functions $w_{h}$ as $v$, passing to the limit we obtain

$$
\tilde{u}+\bar{\psi} \wedge t \geq \bar{\psi} \quad(m, p) \text {-q.e. }
$$

this implies that

$$
\tilde{u} \geq \bar{\psi}-\bar{\psi} \wedge t=(\bar{\psi}-t)^{+} \quad(m, p) \text {-q.e. on the set }\{\bar{\psi}>-\infty\}
$$

on the other hand, $\tilde{u} \geq 0(m, p)$-q.e. as $u \in K^{\prime \prime}\left[\left(\left(\psi_{h}-t\right)^{+}\right)\right]$. We conclude that $u \in K\left((\psi-t)^{+}\right)$and therefore

$$
K^{\prime \prime}\left(\left(\psi_{h}-t\right)^{+}\right) \subseteq K\left((\psi-t)^{+}\right) .
$$

In a similar way on can prove that

$$
K^{\prime \prime}\left(\left(\psi_{h} \wedge t\right)\right) \subseteq K(\psi \wedge t)
$$

LEMMA 3.10. - Let $X$ be a separable metric space, $I$ an open interval of $\mathbb{R}$ (possibly unbounded) and $\left(K_{t}\right)_{t \in I}$ a family of closed subsets of $X$, increasing for $t \in I$ (i.e. $K_{s} \subseteq K_{t}$ whenever $s, t \in I$ and $s \leq t$ ). Then there exists a countable set $T \subseteq I$ such that for every $t \in I \backslash T$ we have

$$
K_{t}=\mathrm{cl}\left(\bigcup_{s<t} K_{s}\right)=\bigcap_{\tau>t} K_{\tau} .
$$

Proof. - See the proof of theorem 2.7 in [9]. 
Proof of theorem 3.1. - Observe that corollary 3.6 gives the equivalence between (3.1) and $\left(3.1^{\prime}\right)$.

Part $I$ Assume that there exists a dense set $D$ in $\mathbb{R}$ such that (3.1) holds for every $t \in D$. Suppose $\psi$ and $\psi_{h}$, for every $h \in \mathbb{N}$, to be defined all over $\mathbb{R}^{n}$ with value $-\infty$ outside $\Omega$. Let us prove that

$$
K\left(\psi_{h}\right) \rightarrow K(\psi) \text { in } W^{m, p},
$$

which implies the convergence of $\left(K_{0}\left(\psi_{h}\right)\right)$ to $K_{0}(\psi)$ in $W_{0}^{m, p}(\Omega)$ by lemma 3.2.

We note that we may assume the obstacles $\psi_{h}$ and $\psi$ also equi-bounded from below on $\Omega^{\prime}$. Indeed, by lemma 3.5 (part $(a) \Rightarrow(b)$ ), (3.2) follows from the convergence

$$
K\left(\psi_{h} \vee s\right) \rightarrow K(\psi \vee s)
$$

for every $s \in \mathbb{R}^{-}$. Observe now that if $s \in \mathbb{R}^{-}$and $h \in \mathbb{N}$, we have

$$
\psi_{h} \vee s=\left[T_{\Omega^{\prime}}\left(\psi_{h} \vee s\right)\right] \vee s
$$

and the corresponding equality for $\psi$. Therefore, in view of lemma 3.5 (part (b) $\Rightarrow(a))$, to get (3.2) it suffices to prove that

$$
K\left(T_{\Omega^{\prime}}\left(\psi_{h} \vee s\right)\right) \rightarrow K\left(T_{\Omega^{\prime}}(\psi \vee s)\right)
$$

for every $s \in \mathbb{R}^{-}$. Hence we may assume that the stronger condition $|\psi|,\left|\psi_{h}\right| \leq M$ on $\Omega^{\prime}$ for every $h \in \mathbb{N}$ is satisfied. Moreover, since the obstacles are equal to $-\infty$ outside a compact subset of $\Omega$, lemma 3.3 allows us to assume that $0<\psi, \psi_{h} \leq M$ on $\Omega^{\prime}$ for every $h \in \mathbb{N}$. For the same reason it is not restrictive to suppose that $0 \in D$.

In order to prove (3.2) we first show that $K(\psi) \subseteq K^{\prime}\left(\left(\psi_{h}\right)\right)$. Fix $\epsilon>0$; by the density of $D$ there exist $t_{0}, t_{1}, \ldots, t_{q}$ in $\mathbb{R}$ such that $0=t_{0}<t_{1}<\cdots<t_{q}=M, t_{i}-t_{i-1}<\epsilon$ for $i=1, \ldots, q$ and $t_{i} \in D$ for $i=0, \ldots, q-1$. For every $h \in \mathbb{N}$ define

$$
\Phi_{h}=\sum_{i=1}^{q}\left(t_{i}-t_{i-1}\right) \mathbf{1}_{\left\{\psi_{h}>t_{i-1}\right\}}, \quad \Phi=\sum_{i=1}^{q}\left(t_{i}-t_{i-1}\right) \mathbf{1}_{\left\{\psi>t_{i-1}\right\}} .
$$

We want to prove that

$$
\begin{aligned}
K(\Phi) & \subseteq K^{\prime}\left(\left(\Phi_{h}\right)\right) \\
& -119-
\end{aligned}
$$




\section{Enrico Vitali}

arguing by induction. Since

$$
\Phi_{h} \wedge t_{1}=t_{1} \mathbf{1}_{\left\{\psi_{h}>0\right\}}, \quad \Phi \wedge t_{1}=t_{1} \mathbf{1}_{\{\psi>0\}},
$$

condition (3.1) for $t=t_{0}=0$ immediately gives the first step, i.e. $K\left(\Phi \wedge t_{1}\right) \subseteq K^{\prime}\left(\left(\Phi_{h} \wedge t_{1}\right)\right)$. Assume now that

$$
K\left(\Phi \wedge t_{i}\right) \subseteq K^{\prime}\left(\left(\Phi_{h} \wedge t_{i}\right)\right)
$$

for some $i=1, \ldots, q-1$. Since

$$
\begin{aligned}
\left(\left(\Phi_{h} \wedge t_{i+1}\right)-t_{i}\right)^{+} & =\left(t_{i+1}-t_{i}\right) \mathbf{1}_{\left\{\psi_{h}>t_{i}\right\}} \\
\left(\left(\Phi \wedge t_{i+1}\right)-t_{i}\right)^{+} & =\left(t_{i+1}-t_{i}\right) \mathbf{1}_{\left\{\psi>t_{i}\right\}},
\end{aligned}
$$

we have, by assumption, that

$$
K\left[\left(\left(\Phi \wedge t_{i+1}\right)-t_{i}\right)^{+}\right] \subseteq K^{\prime}\left[\left(\left(\left(\Phi_{h} \wedge t_{i+1}\right)-t_{i}\right)^{+}\right)_{h}\right] .
$$

This inclusion, together with (3.4) and lemma 3.8, gives

$$
K\left(\Phi \wedge t_{i+1}\right) \subseteq K^{\prime}\left(\left(\Phi_{h} \wedge t_{i+1}\right)\right) .
$$

Thus we have proved the inductive step, hence inclusion (3.3).

We are now in a position to prove that $K(\psi) \subseteq K^{\prime}\left(\left(\psi_{h}\right)\right)$. Let $u \in K(\psi)$ and $\varphi \in C_{0}^{\infty}\left(\mathbb{R}^{n}\right)$ with $\varphi=1$ on $\Omega^{\prime}$. Then $u+\epsilon \varphi \in K(\Phi)$ and, by (3.3), $u+\epsilon \varphi \in K^{\prime}\left(\left(\Phi_{h}\right)\right)$; since $\Phi_{h} \geq \psi_{h}$ for every $h \in \mathbb{N}$, we have $u+\epsilon \varphi \in K^{\prime}\left(\left(\psi_{h}\right)\right)$. We conclude that $K^{\prime}\left(\left(\psi_{h}\right)\right)$ contains $u$ as it is closed in $W^{m, p}$ and $\epsilon>0$ is arbitrary. Therefore $K(\psi) \subseteq K^{\prime}\left(\left(\psi_{h}\right)\right)$.

Now let us prove that $K^{\prime \prime}\left(\left(\psi_{h}\right)\right) \subseteq K(\psi)$. Let $u \in K^{\prime \prime}\left(\left(\psi_{h}\right)\right)$ : there exist a strictly increasing sequence of positive intergers $\left(h_{k}\right)$ and a sequence $\left(u_{k}\right)$ converging to $u$ weakly in $W^{m, p}$, such that $u_{k} \in K\left(\psi_{h_{k}}\right)$ for every $k \in \mathbb{N}$. If $\varphi$ is as above, for every fixed $t \in D$ we have

$$
\begin{gathered}
u_{k}-t \varphi \in K\left(\chi_{\left\{\psi_{h_{k}}>t\right\}}\right) \quad \text { for every } k \in \mathbb{N}, \\
\left(u_{k}-t \varphi\right) \text { converges to } u-t \varphi \text { weakly in } W^{m, p} .
\end{gathered}
$$

Our assumption, in the form $\left(3.1^{\prime}\right)$, together with lemma 3.2, implies that $u-t \varphi \in K\left(\chi_{\{\psi>t\}}\right)$, hence $\tilde{u} \geq t(m, p)$-q.e. on the set $\{\psi>t\}$. Since $t$ is arbitrary in $D$ we easily obtain that $u \in K(\psi)$. Therefore $K^{\prime \prime}\left(\left(\psi_{h}\right)\right) \subseteq K(\psi)$. We conclude that (3.2) holds. 
Part II Assume that

$$
K_{0}\left(\psi_{h}\right) \rightarrow K_{0}(\psi) \text { in } W_{0}^{m, p}(\Omega)
$$

Let us prove that there exists a subset $D$ of $\mathbb{R}$ such that $\mathbb{R} \backslash D$ is countable and condition (3.1) holds for every $t \in D$. We point out that the following proof does not use the assumption of boundedness of the obstacles.

By lemma 3.2 we have

$$
K\left(\psi_{h}\right) \rightarrow K(\psi) \text { in } W^{m, p}
$$

Let $s, t \in \mathbb{R}^{+}$with $s<t$ and define

$$
\begin{aligned}
g_{h} & =(t-s)^{-1}\left[\left(\psi_{h} \wedge t\right)-s\right]^{+} \\
g & =(t-s)^{-1}[(\psi \wedge t)-s]^{+}
\end{aligned}
$$

for every $h \in \mathbb{N}$. By lemma 3.9, $\left(K\left(g_{h}\right)\right)$ converges to $K(g)$. Moreover

$$
\begin{aligned}
& \mathbf{1}_{\left\{\psi_{h}>t\right\}} \leq g_{h} \leq \mathbf{1}_{\left\{\psi_{h}>s\right\}} \\
& \mathbf{1}_{\{\psi>t\}} \leq g \leq \mathbf{1}_{\{\psi>s\}}
\end{aligned}
$$

so that

$$
\begin{aligned}
K\left(\mathbf{1}_{\{\psi>s\}}\right) & \subseteq K^{\prime}\left(\left(\mathbf{1}_{\left\{\psi_{h}>t\right\}}\right)\right) \\
K^{\prime \prime}\left(\left(\mathbf{1}_{\left\{\psi_{h}>s\right\}}\right)\right) & \subseteq K\left(\mathbf{1}_{\{\psi>t\}}\right) .
\end{aligned}
$$

Hence, for every $t \in \mathbb{R}^{+}$,

$$
\begin{aligned}
\operatorname{cl}_{W^{m, p}}\left[\bigcup_{s<t} K\left(\mathbf{1}_{\{\psi>s\}}\right)\right] & \subseteq K^{\prime}\left(\left(\mathbf{1}_{\left\{\psi_{h}>t\right\}}\right)\right) \\
& \subseteq K^{\prime \prime}\left(\left(\mathbf{1}_{\left\{\psi_{h}>t\right\}}\right)\right) \subseteq \bigcap_{\tau>t} K\left(\mathbf{1}_{\{\psi>\tau\}}\right) .
\end{aligned}
$$

Apply now lemma 3.10: there exists a countable set $T_{0} \subseteq \mathbb{R}^{+}$such that, for every $t \in \mathbb{R}^{+} \backslash T_{0}$,

$$
K^{\prime}\left(\left(\mathbf{1}_{\left\{\psi_{h}>t\right\}}\right)\right)=K^{\prime \prime}\left(\left(\mathbf{1}_{\left\{\psi_{h}>t\right\}}\right)\right)=K\left(\mathbf{1}_{\{\psi>t\}}\right)
$$

i.e.

$$
\begin{aligned}
K\left(\mathbf{1}_{\left\{\psi_{h}>t\right\}}\right) \rightarrow & K\left(\mathbf{1}_{\{\psi>t\}}\right) \text { in } W^{m, p} \\
& -121-
\end{aligned}
$$


By lemma 3.2 we conclude that

$$
K_{0}\left(\mathbf{1}_{\left\{\psi_{h}>t\right\}}\right) \rightarrow K_{0}\left(\mathbf{1}_{\{\psi>t\}}\right) \text { in } W_{0}^{m, p}(\Omega)
$$

for every $t \in \mathbb{R}^{+} \backslash T_{0}$.

Since for every $\lambda \in \mathbb{R}$, condition (3.5) and lemma 3.3 imply that

$$
K\left(\psi_{h}+\lambda\right) \rightarrow K(\psi+\lambda) \quad \text { in } W^{m, p}
$$

what we have just proved allows us to say that for every $\lambda \in \mathbb{R}$ the set $T_{\lambda}$ of those $t \in \mathbb{R}$ with $t \geq-\lambda$ for which (3.1) does not hold, is at most countable. Now it suffices to make $\lambda$ vary in $\mathbb{Z}$.

\section{Convergence of obstacles and level sets:}

The general case

In this section we extend the result of theorem 3.1 to the case of obstacles dominated by a sequence of functions strongly converging in $W_{0}^{m, p}(\Omega)$.

THEOREM 4.1. - Let $\left(\psi_{h}\right)$ be a sequence of functions from $\Omega$ into $\overline{\mathbb{R}}$ and let $\psi$ be a function from $\Omega$ into $\overline{\mathbb{R}}$. Then

$$
K_{0}\left(\psi_{h}\right) \rightarrow K_{0}(\psi) \quad \text { in } W_{0}^{m, p}(\Omega) \quad \text { and } \quad K_{0}(\psi) \neq \emptyset
$$

if and only if the following conditions (a) and (b) are satisfied:

(a) there exists a dense set $D$ in $\mathbb{R}$ and a set $\mathcal{F} \subseteq \mathcal{A}(\Omega)$, cofinal in $\Omega$, such that

$$
K_{0}\left(\mathbf{1}_{\left\{\psi_{h}>t\right\} \cap B}\right) \rightarrow K_{0}\left(\mathbf{1}_{\{\psi>t\} \cap B}\right) \text { in } W_{0}^{m, p}(\Omega)
$$

for every $t \in D$ and every $B \in \mathcal{F}$;

(b) there exist a sequence $\left(w_{h}\right)$ in $W_{0}^{m, p}(\Omega)$ and an index $k \in \mathbb{N}$ such that $\left(w_{h}\right)$ converges strongly in $W_{0}^{m, p}(\Omega)$ and $w_{h} \in K_{0}\left(\psi_{h}\right)$ for every $h \geq k$.

Moreover, if $B \Subset \Omega,(4.1)$ is equivalent to

$$
K_{0}\left(\chi_{\left\{\psi_{h}>t\right\} \cap B}\right) \rightarrow K_{0}\left(\chi_{\{\psi>t\} \cap B}\right) \text { in } W_{0}^{m, p}(\Omega) .
$$

Finally, we may assume that $\mathbb{R} \backslash D$ is countable and that $B \Subset \Omega$ for every $B \in \mathcal{F}$. 
Before proving this theorem we state, without proof, a localization result which can be obtained as theorem 2.7 in [9], and for which we use the technical notion of rich set introduced in section 1.

Proposition 4.2. - Let $\left(\psi_{h}\right)$ and $\psi$ be as in theorem 4.1. Suppose that $K_{0}(\psi) \neq \emptyset$ and that

$$
K_{0}\left(\psi_{h}\right) \rightarrow K_{0}(\psi) \quad \text { in } W_{0}^{m, p}(\Omega)
$$

Then there exists a set $\mathcal{R} \subseteq \mathcal{A}(\Omega)$, rich in $\mathcal{A}(\Omega)$, such that

$$
K_{0}\left(\psi_{h}, A\right) \rightarrow K_{0}(\psi, A) \quad \text { in } W_{0}^{m, p}(\Omega)
$$

for every $A \in \mathcal{R}$.

Besides, we need one more lemma, for which we refer to lemma 4.7 in [9].

LEMMA 4.3. - Let $\psi$ and $\left(\psi_{h}\right)$ be a function and a sequence of functions, respectively, from $\mathbb{R}^{n}$ into $\overline{\mathbb{R}}$; let $T$ be an unbounded subset of $\mathbb{R}^{+}$. Assume that

$$
K\left(\psi_{h} \wedge t\right) \rightarrow K(\psi \wedge t) \quad \text { in } W^{m, p}
$$

for every $t \in T$, and that

$$
\lim _{t \rightarrow+\infty} \limsup _{h \rightarrow+\infty} B_{m, p}\left(\left(\psi_{h}-t\right)^{+}\right)=0 .
$$

Then

$$
K\left(\psi_{h}\right) \rightarrow K(\psi) \quad \text { in } W^{m, p}
$$

Proof of theorem 4.1. - The equivalence between (4.1) and $\left(4.1^{\prime}\right)$ for $B \Subset \Omega$ comes from corollary 3.6 , while, by proposition 4.2 , we may suppose that $B \Subset \Omega$ if $B \in \mathcal{F}$.

Assume that $\left(K_{0}\left(\psi_{h}\right)\right)$ converges to $K_{0}(\psi)$ and that $K_{0}(\psi) \neq \emptyset$. Let us prove that condition (a) holds with a set $D$ whose complement in $\mathbb{R}$ is at most countable. By proposition 4.2 there exists a countable set $\mathcal{F} \subseteq \mathcal{A}(\Omega)$, cofinal in $\Omega$, such that $B \Subset \Omega$ and

$$
K_{0}\left(\psi_{h}, B\right) \rightarrow K_{0}(\psi, B)
$$

for every $B \in \mathcal{F}$. Since part II in the proof of theorem (3.1) does not depend, as we remarked, on the boundedness of the obstacles, we obtain 
that for every $B \in \mathcal{F}$ there exists a set $D_{B} \subseteq \mathbb{R}$ such that $\mathbb{R} \backslash D_{B}$ is countable and

$$
K_{0}\left(\mathbf{1}_{\left\{\psi_{h}>t\right\} \cap B}\right) \rightarrow K_{0}\left(\mathbf{1}_{\{\psi>t\} \cap B}\right)
$$

for every $t \in D_{B}$. Since $\mathcal{F}$ is countable, we get condition (a) with a set $D$ such that $\mathbb{R} \backslash D$ is at most countable.

Condition (b) is an immediate consequence of definition 1.7 of Mosco convergence.

Assume now (a) and (b). As observed above, we may suppose that if $B \in \mathcal{F}$ then $B \Subset \Omega$. First of all we want to prove that

$$
K\left(\psi_{h}, B\right) \rightarrow K(\psi, B) \quad \text { in } W^{m, p}
$$

for every $B \in \mathcal{F}$.

We claim that (4.3) is implied by condition (b) together with the following one

$$
K\left(\psi_{h} \wedge t, B\right) \rightarrow K(\psi \wedge t, B)
$$

for every $t \in \mathbb{R}^{+}$. To prove this, it suffices to show that (b) implies condition (4.2) of lemma 4.3. Let $\left(w_{h}\right)$ and $k$ be as in (b); denote by $w$ the limit of $\left(w_{h}\right)$ in $W_{0}^{m, p}(\Omega)$ and define $f=\left(J_{m}\right)^{-1} w$ and $f_{h}=\left(J_{m}\right)^{-1} w_{h}$, for every $h \in \mathbb{N}$ (here we consider $w$ and $w_{h}$ as functions of $W^{m, p}$ with value zero outside $\Omega$ ). For every $t \in \mathbb{R}^{+}$and $h \geq k$ we have

$$
\begin{aligned}
\limsup _{h \rightarrow+\infty} B_{m, p}\left(\left(\psi_{h}-t\right)^{+}\right) & \leq \lim _{h \rightarrow+\infty}\left\|G_{m} \star\left(f_{h}-t\right)^{+}\right\|_{m, p}^{p} \\
& =\left\|(f-t)^{+}\right\|_{L^{p}}^{p}
\end{aligned}
$$

we obtain (4.2) taking the limit as $t \rightarrow+\infty$.

Now (4.4) follows from condition (a), lemma 3.2 and theorem 3.1 applied to the sequence $\left(T_{B}\left(\psi_{h} \wedge t\right)\right)$, taking into account that

$$
\begin{aligned}
\left\{T_{B}\left(\psi_{h} \wedge t\right)>\tau\right\} & =\left\{\psi_{h}>\tau\right\} \cap B \\
\left\{T_{B}(\psi \wedge t)>\tau\right\} & =\{\psi>\tau\} \cap B
\end{aligned}
$$

for $\tau<t$. We have thus proved (4.3).

From (4.3) and lemma 3.2 we obtain

$$
\begin{aligned}
K_{0}\left(\psi_{h}, B\right) \rightarrow & K_{0}(\psi, B) \text { in } W_{0}^{m, p}(\Omega) \\
& -124-
\end{aligned}
$$


for every $B \in \mathcal{F}$. Since $\mathcal{F}$ is cofinal in $\Omega$ and $(b)$ holds, corollary 2.13 and (4.5) yield

$$
K_{0}^{\prime}\left(\left(\psi_{h}\right)\right)=\bigcap_{B \in \mathcal{F}} K_{0}^{\prime}\left(\left(\psi_{h}\right), B\right) \supseteq \bigcap_{B \in \mathcal{F}} K_{0}(\psi, B)=K_{0}(\psi),
$$

hence $K_{0}(\psi) \subseteq K_{0}^{\prime}\left(\left(\psi_{h}\right)\right)$.

Finally, by (4.5)

$$
K_{0}^{\prime \prime}\left(\left(\psi_{h}\right)\right) \subseteq \bigcap_{B \in \mathcal{F}} K_{0}^{\prime \prime}\left(\left(\psi_{h}\right), B\right) \subseteq \bigcap_{B \in \mathcal{F}} K_{0}(\psi, B)=K_{0}(\psi),
$$

hence $K_{0}^{\prime \prime}\left(\left(\psi_{h}\right)\right) \subseteq K_{0}(\psi)$. We conclude that

$$
K_{0}\left(\psi_{h}\right) \rightarrow K_{0}(\psi) \quad \text { in } W_{0}^{m, p}(\Omega)
$$

and consequently $K_{0}(\psi) \neq \emptyset$ by condition (b).

The next theorem presents a simple situation in which condition (b) of theorem 4.1 is satisfied without requiring that the obstacles are bounded from above. In the following characterization only the level sets of the obstacles are involved.

THEOREM 4.4.- Let $\left(\psi_{h}\right)$ be a sequence of functions from $\Omega$ into $\overline{\mathbb{R}}$ and let $\psi$ be a function from $\Omega$ into $\overline{\mathbb{R}}$. Assume, in addition, the existence of a set $\Omega^{\prime} \Subset \Omega$ such that $\psi, \psi_{h} \leq 0(m, p)$-q.e. on $\Omega \backslash \Omega^{\prime}$ for every $h \in \mathbb{N}$. Then

$$
K_{0}\left(\psi_{h}\right) \rightarrow K_{0}(\psi) \quad \text { in } W_{0}^{m, p}(\Omega) \quad \text { and } \quad K_{0}(\psi) \neq \emptyset
$$

if and only if the following conditions $\left(a^{\prime}\right)$ and $\left(b^{\prime}\right)$ are satisfied:

$\left(a^{\prime}\right)$ there exists a dense set $D$ in $\mathbb{R}$ and a set $\mathcal{F} \subseteq \mathcal{A}(\Omega)$, cofinal in $\Omega$, such that

$$
K_{0}\left(\mathbf{1}_{\left\{\psi_{h}>t\right\} \cap B}\right) \rightarrow K_{0}\left(\mathbf{1}_{\{\psi>t\} \cap B}\right) \text { in } W_{0}^{m, p}(\Omega)
$$

for every $t \in D$ and every $B \in \mathcal{F}$;

(b') $\lim _{t \rightarrow+\infty} \limsup _{h \rightarrow+\infty} \int_{t}^{+\infty} C_{m, p}\left(\left\{\psi_{h}>s\right\}\right)(s-t)^{p-1} \mathrm{~d} s=0$.

Proof. - By theorem 2.2, $\left(\mathrm{b}^{\prime}\right)$ is equivalent to

$$
\lim _{t \rightarrow+\infty} \limsup _{h \rightarrow+\infty} B_{m, p}\left(\left(\psi_{h}-t\right)^{+}\right)=0 .
$$


As shown in the proof of theorem 4.1, this is implied by condition (b) (of theorem 4.1).

Assume now $\left(\mathrm{a}^{\prime}\right)$ and $\left(\mathrm{b}^{\prime}\right)$, and let us prove that (b) holds. Since $\psi, \psi_{h} \leq 0$ outside $\Omega^{\prime}$, in view of theorem 4.1 we obtain

$$
K_{0}\left(\psi_{h}^{+} \wedge t\right) \rightarrow K_{0}\left(\psi^{+} \wedge t\right) \text { in } W_{0}^{m, p}(\Omega)
$$

for every $t \in \mathbb{R}^{+}$. Therefore, by lemma 3.2,

$$
K\left(\psi_{h}^{+} \wedge t\right) \rightarrow K\left(\psi^{+} \wedge t\right) \quad \text { in } W^{m, p} .
$$

On account of (4.6), we can apply lemma 4.3 and conclude that

$$
K\left(\psi_{h}^{+}\right) \rightarrow K\left(\psi^{+}\right) .
$$

Furthermore, since each obstacle $\psi_{h}$ is non-positive outside $\Omega^{\prime}$, from (4.6) we easily get that there exists a sequence $\left(w_{h}\right)$ bounded in $W^{m, p}$ and such that $\tilde{w}_{h} \geq \psi_{h}^{+}(m, p)$-q.e. for $h$ sufficiently large. We can extract a subsequence $\left(w_{h_{k}}\right)$ which converges weakly in $W^{m, p}$ to a function $w$. Therefore $w \in K^{\prime \prime}\left(\left(\psi_{h}^{+}\right)\right)$and, by (4.7), $w \in K^{\prime}\left(\left(\psi_{h}^{+}\right)\right)$. It follows that $K^{\prime}\left(\left(\psi_{h}\right)\right)$ is not empty, and this implies condition (b) by definition of Mosco convergence.

Now, it only remains to apply theorem 4.1 .

\section{Convergence of level sets and convergence of capacities}

In this section we consider the sequence of convex sets $\left(K_{0}\left(\mathbf{1}_{E_{h}}\right)\right)$ in $W_{0}^{m, p}(\Omega)$ associated to a given sequence $\left(E_{h}\right)$ of subsets of $\Omega$, and we express its Mosco convergence to a set of the same form through a convergence condition for the capacities of the intersections $E_{h} \cap A$ with a rich family of open sets $A$ (theorem 5.1). Afterwards we link this result with the one of the previous section by taking as $\left(E_{h}\right)$ the level sets of a sequence of obstacles (theorem 5.12).

In this section only the case $p=2$ will be studied.

THEOREM 5.1. - Let $\left(E_{h}\right)$ be a sequence of subsets of $\Omega$ and let $E$ be a subset of $\Omega$. Assume, in addition, that there exists a set $\Omega^{\prime} \Subset \Omega$ which contains $E$ and the sequence $\left(E_{h}\right)$. Then

$$
\begin{aligned}
K_{0}\left(\mathbf{1}_{E_{h}}\right) \rightarrow & K_{0}\left(\mathbf{1}_{E}\right) \quad \text { in } H_{0}^{m}(\Omega) \\
& -126-
\end{aligned}
$$


if and only if there exists a set $\mathcal{R} \subseteq \mathcal{A}(\Omega)$, rich in $\mathcal{A}(\Omega)$, such that

$$
\lim _{h \rightarrow+\infty} C_{m, 2}\left(E_{h} \cap A\right)=C_{m, 2}(E \cap A)
$$

for every $A \in \mathcal{R}$.

Remark 5.2. - We observe that the existence of a set $\mathcal{R} \subseteq \mathcal{A}(\Omega)$, rich in $\mathcal{A}(\Omega)$, such that (5.1) holds for every $A \in \mathcal{R}$ is equivalent to the existence of a set $\mathcal{D} \subseteq \mathcal{A}(\Omega)$, dense in $\mathcal{A}(\Omega)$, such that (5.1) holds for every $A \in \mathcal{D}$. Indeed, one implication is obvious; conversely, define

$$
\begin{aligned}
\alpha^{\prime}(A) & =\liminf _{h \rightarrow+\infty} C_{m, 2}\left(E_{h} \cap A\right) \\
\alpha^{\prime \prime}(A) & =\limsup _{h \rightarrow+\infty} C_{m, 2}\left(E_{h} \cap A\right) \\
\alpha(A) & =C_{m, 2}(E \cap A)
\end{aligned}
$$

for every $A \in \mathcal{A}(\Omega)$. The functions $\alpha, \alpha^{\prime}$ and $\alpha^{\prime \prime}$ are increasing on $\mathcal{A}(\Omega)$ and coincide on a dense set, therefore they coincide on a rich set in $\mathcal{A}(\Omega)$ (see, for example, proposition 4.8 in [16]).

The idea to derive the convergence of $\left(K_{0}\left(1_{E_{h}}\right)\right)$ to $K_{0}\left(\mathbf{1}_{E}\right)$ from the validity of (5.1) for a rich family of open sets $A$, may be sketched as follows. In a standard way we change the problem of Mosco convergence into a problem of $\Gamma$-convergence (see definitions below) for an associated sequence of functionals. By means of a compactness theorem we get a $\Gamma$ limit, which can be identified as the functional corresponding to $E$ thanks to the possibility of recognizing $E$ from the knowledge of $C_{m, 2}(E \cap A)$ for sufficiently many open sets $A$.

Let us briefly recall the notion of $\Gamma$-convergence. Let $(X, \tau)$ be a topological space satisfying the first axiom of countability.

Definition 5.3. - Let $\left(F_{h}\right)$ be a sequence of functions from $X$ into $\bar{R}$, $F$ a function from $X$ into $\mathbb{R}$ and $u \in X$. We say that $\left(F_{h}\right) \Gamma(\tau)$-converges to $F$ in $u$, and we write

$$
F(u)=\Gamma(\tau) \lim _{\substack{h \rightarrow+\infty \\ v \rightarrow u}} F_{h}(v)
$$

if the following conditions are satisfied:

(i) for every sequence $\left(u_{h}\right)$ converging to $u$ in $\tau$

$$
F(u) \leq \liminf _{h \rightarrow+\infty} F_{h}\left(u_{h}\right)
$$




\section{Enrico Vitali}

(ii) there exists a sequence $\left(u_{h}\right)$ converging to $u$ in $\tau$ such that

$$
F(u) \geq \limsup _{h \rightarrow+\infty} F_{h}\left(u_{h}\right)
$$

$\left(F_{h}\right)$ is said to $\Gamma(\tau)$-converge to $F$ if $\left(F_{h}\right) \Gamma(\tau)$-converges to $F$ in every point $u$ of $X$.

For a complete treatment of this kind of convergence see, for instance, [15] and [3]. Here we limit ourselves to state one of the most significant variational properties of $\Gamma$-convergence (see, corollary 2.4 in [15]).

THEOREM 5.4. - Let $\left(F_{h}\right)$ be a sequence of functions from $X$ into $\overline{\mathbb{R}}$ which $\Gamma(\tau)$-converges to a function $F$. Suppose that there exists a $\tau$-compact subset $K$ of $X$ such that

$$
\inf _{u \in X} F_{h}(u)=\inf _{u \in K} F_{h}(u)
$$

for every $h \in \mathbb{N}$. Then $F$ attains its minimum in $X$ and

$$
\lim _{h \rightarrow+\infty} \inf _{u \in X} F_{h}(u)=\min _{u \in X} F(u) .
$$

Furthermore, if $u_{h}$ is a minimum point of $F_{h}$ in $X$ for every $h \in \mathbb{N}$, and $\left(u_{h}\right)$ converges to a point $u$ in $\tau$, then $u$ is a minimum point of $F$ in $X$.

For any subset $C$ of $H^{m}$ let us denote by $I_{C}$ the indicator function of $C$ in $H^{m}$, i.e. the mapping which takes the value 0 on $C$ and $+\infty$ on $H^{m} \backslash C$. Moreover, by $H_{0}^{0}(\Omega)$ we shall mean the space $L^{2}(\Omega)$. The following proposition has a simple proof which we omit.

Proposition 5.5. - Let $K$ and $\left(K_{h}\right)$ be a subset and a sequence of subsets, respectively, of $H^{m}$. Then

$$
K_{h} \rightarrow K \quad \text { in } H^{m}
$$

if and only if

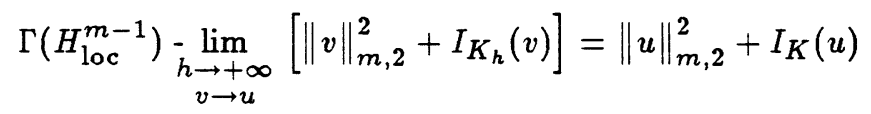

where $\Gamma\left(H_{\mathrm{loc}}^{m-1}\right)$ means that the $\Gamma$-convergence is considered with respect to the topology of $H_{\mathrm{loc}}^{m-1}\left(\mathbb{R}^{n}\right)$ on the space $H^{m}\left(\mathbb{R}^{n}\right)$. 
For every $B \in \mathcal{B}$ and $u \in H^{m}$ define

$$
F(u, B)=\sum_{|\alpha| \leq m} \int_{B}\left|D^{\alpha} u\right|^{2} \mathrm{~d} x .
$$

Moreover, given a subset $E$ of $\mathbb{R}^{n}$, we define

$$
\begin{gathered}
G_{E}(u, B)=I_{K\left(\mathbf{1}_{E \cap B}\right)}(u)= \begin{cases}0 & \text { if } \tilde{u} \geq \mathbf{1}_{E \cap B}(m, 2) \text {-q.e. } \\
+\infty & \text { otherwise in } H^{m},\end{cases} \\
H_{E}(u, B)=I_{K\left(\mathbf{1}_{E}, B\right)}(u)= \begin{cases}0 & \text { if } \tilde{u} \geq \mathbf{1}_{E}(m, 2) \text {-q.e. on } B \\
+\infty & \text { otherwise in } H^{m}\end{cases}
\end{gathered}
$$

for every $B \subseteq \mathbb{R}^{n}$ and $u \in H^{m}$. A simple check shows that $H_{E}$ satisfies the following properties, which we state for a functional $H: H^{m} \times \mathcal{B} \rightarrow$ $[0,+\infty]$;

(i) for every $A \in \mathcal{A}$, the function $H(\cdot, A)$ is lower semicontinuous in $H^{m}$;

(ii) for every $u \in H^{m}$, the function $H(u, \cdot)$ is a Borel measure on $\mathbb{R}^{n}$;

(iii) if $u, v \in H^{m}, A \in \mathcal{A}$ and $u=v$ a.e. on $A$, then $H(u, A)=H(v, A)$;

(iv) if $u, v \in H^{m}, A \in \mathcal{A}$ and $u \leq v$ a.e. on $A$, then $H(u, A) \geq H(v, A)$.

Let us denote by $\left(H^{m}\right)^{+}$the cone of non-negative functions of $H^{m}$. From theorem 1 in [24] and theorem 3.3 in [14] we derive the next theorem.

THEOREM 5.6. - Let $\left(E_{h}\right)$ be a sequence of subsets of $\mathbb{R}^{n}$. Then there exist a subsequence $\left(E_{\sigma(h)}\right)$ of $\left(E_{h}\right)$, a functional $G: H^{m} \times \mathcal{A} \rightarrow[0,+\infty]$ and a set $\mathcal{R}^{\prime} \subseteq \mathcal{A}$, rich in $\mathcal{A}$, such that

$$
\begin{array}{cr}
{\left[F(\cdot, A)+G_{E_{\sigma(h)}}(\cdot, A)\right]} & \Gamma\left(H_{\text {loc }}^{m-1}\right) \text {-converges to }[F(\cdot, A)+G(\cdot, A)] \\
{\left[F\left(\cdot, \mathbb{R}^{n}\right)+G_{E_{\sigma(h)}}(\cdot, A)\right]} & \Gamma\left(H_{\text {loc }}^{m-1}\right) \text {-converges to }\left[F\left(\cdot, \mathbb{R}^{n}\right)+G(\cdot, A)\right]
\end{array}
$$

for every $A \in \mathcal{R}^{\prime}$. Moreover, there exists a functional $H: H^{m} \times \mathcal{B} \rightarrow$ $[0,+\infty]$ satisfying (i) to (iv) above and such that (5.2) and (5.3) hold with $\left(G_{E_{\sigma(h)}}\right)$ and $G$ replaced by $\left(H_{E_{\sigma(h)}}\right)$ and $H$, respectively. Finally,

$$
G(u, A)= \begin{cases}H(u, A) & \text { if } u \in\left(H^{m}\right)^{+} \\ +\infty & \text { otherwise in } H^{m}\end{cases}
$$

for every $u \in H^{m}$ and every $A \in \mathcal{A}$. 


\section{Proof}

Step 1 Here we prove the existence of a subsequence $\left(E_{\sigma(h)}\right)$ of $\left(E_{h}\right)$, a functional $G: H^{m} \times \mathcal{A} \rightarrow[0,+\infty]$, and a set $\mathcal{R}_{1} \subseteq \mathcal{A}$, rich in $\mathcal{A}$, such that (5.2) holds for every $A \in \mathcal{R}_{1}$. This step is rather standard (see, for example, theorem 15.8 in [10] or theorem 4.15 in [13]) and it relies only on the property that $\left(G_{E_{h}}\right)$ is a sequence of functionals increasing in the second variable.

Let $M^{\prime}$ and $M^{\prime \prime}$ be the functionals defined, for every $u \in H^{m}$ and for every pair $\omega, A$ of open subsets of $\mathbb{R}^{n}$ with $A \subseteq \omega$, through the equations

$$
\begin{aligned}
& M^{\prime}(u, \omega, A)=\inf \left\{\liminf _{h \rightarrow+\infty}\left[F\left(u_{h}, \omega\right)+G_{E_{h}}\left(u_{h}, A\right)\right] \mid u_{h} \rightarrow u \text { in } H_{\text {loc }}^{m-1}\right\} \\
& M^{\prime \prime}(u, \omega, A)=\inf \left\{\limsup _{h \rightarrow+\infty}\left[F\left(u_{h}, \omega\right)+G_{E_{h}}\left(u_{h}, A\right)\right] \mid u_{h} \rightarrow u \text { in } H_{\text {loc }}^{m-1}\right\} .
\end{aligned}
$$

It is easy to see, by a diagonal argument, that the infima in (5.5) and (5.6) are actually minima. Moreover, $M^{\prime}(\cdot, \omega, A)$ and $M^{\prime \prime}(\cdot, \omega, A)$ are lower semicontinuous on $H^{m}$ with the topology of $H_{\text {loc }}^{m-1}$ (see, for example, proposition 1.8 in [15]).

Fix a countable dense subset $\mathcal{D}$ of $\mathcal{A}$. Applying a general compactness theorem with respect to $\Gamma$-convergence (see, for instance, proposition 3.1 in [15]) and a diagonal process, we can find a subsequence $\left(E_{\sigma(h)}\right)$ of $\left(E_{h}\right)$ such that the sequence $\left[F(\cdot, A)+G_{E_{\sigma(h)}}(\cdot, A)\right]$ has a $\Gamma\left(H_{\text {loc }}^{m-1}\right)$-limit for every $A \in \mathcal{D}$.

If $M^{\prime}$ and $M^{\prime \prime}$ are the functionals defined by (5.5) and (5.6), with $\left(E_{h}\right)$ replaced by $\left(E_{\sigma(h)}\right)$, then $M^{\prime}(u, A, A)=M^{\prime \prime}(u, A, A)$ for every $u \in H^{m}$ and $A \in \mathcal{D}$. Thus

$$
\sup _{\substack{A^{\prime} \in \mathcal{A} \\ A^{\prime} \Subset A}} M^{\prime}\left(u, A^{\prime}, A^{\prime}\right)=\sup _{\substack{A^{\prime} \in \mathcal{A} \\ A^{\prime} \Subset A}} M^{\prime \prime}\left(u, A^{\prime}, A^{\prime}\right)
$$

for every $A \in \mathcal{A}$. Besides, since the functions $A \mapsto M^{\prime}(u, A, A)$ and $A \mapsto M^{\prime \prime}(u, A, A)$ are increasing on $\mathcal{A}$ for every $u \in H^{m}$, while $M^{\prime}(\cdot, A, A)$ and $M^{\prime \prime}(\cdot, A, A)$ are lower semicontinuous in $H^{m}$ for every $A \in \mathcal{A}$, we can apply proposition 14.14 in [10] (or argue as in proposition 1.14 of [13]). It 
follows that there exists a set $\mathcal{R}_{1} \subseteq \mathcal{A}$, rich in $\mathcal{A}$, such that

$$
\begin{gathered}
M^{\prime}(u, A, A)=\sup _{\substack{A^{\prime} \in \mathcal{A} \\
\boldsymbol{A}^{\prime} \Subset A}} M^{\prime}\left(u, A^{\prime}, A^{\prime}\right), \\
M^{\prime \prime}(u, A, A)=\sup _{\substack{A^{\prime} \in \mathcal{A} \\
\boldsymbol{A}^{\prime} \Subset \boldsymbol{A}}} M^{\prime \prime}\left(u, A^{\prime}, A^{\prime}\right)
\end{gathered}
$$

for every $u \in H^{m}$ and $A \in \mathcal{R}_{1}$. Defining $M(u, A)$, for every $u \in H^{m}$ and every $A \in \mathcal{A}$, as the common value of both sides in (5.7), and setting $G(u, A)=M(u, A)-F(u, A)$, from (5.7) and (5.8) we conclude that (5.2) holds for every $A \in \mathcal{R}_{1}$.

Step 2 It is rapidly seen that we can apply to the sequence $\left(H_{E_{\sigma(h)}}\right)$ an argument quite similar to the proofs of theorem 1 in [24] and theorem 3.3 in [14]. Thus, passing, if necessary, to a further subsequence, there exist a functional $H: H^{m} \times \mathcal{B} \rightarrow[0,+\infty]$, satisfying (i), (ii) and (iii) above, and a set $\mathcal{R}_{2} \subseteq \mathcal{A}$, rich in $\mathcal{A}$, such that

$$
\left[F(\cdot, A)+H_{E_{\sigma(h)}}(\cdot, A)\right] \quad \Gamma\left(H_{\text {loc }}^{m-1}\right) \text {-converges to }[F(\cdot, A)+H(\cdot, A)]
$$

for every $A \in \mathcal{R}_{2}$. Property (iv) can be obtained as in theorem 2 of [24].

Step 3 Let us prove that the functionals $G$ and $H$ just obtained satisfy (5.4) for every $u \in H^{m}$ and every $A \in \mathcal{A}$. To this aim, for every $u \in H^{m}$ and $\omega, A \in \mathcal{A}$ with $A \subseteq \omega$, we set

$$
\begin{aligned}
G^{\prime}(u, \omega, A) & =M^{\prime}(u, \omega, A)-F(u, \omega) \\
G^{\prime \prime}(u, \omega, A) & =M^{\prime \prime}(u, \omega, A)-F(u, \omega)
\end{aligned}
$$

where $M^{\prime}$ and $M^{\prime \prime}$ are defined in (5.5) and (5.6) (for the sequence $\left(G_{E_{\sigma(h)}}\right)$ ). In the same way we introduce $H^{\prime}(u, \omega, A)$ and $H^{\prime \prime}(u, \omega, A)$ from the sequence $\left(H_{E_{\sigma(h)}}\right)$. The functionals $G^{\prime}, G^{\prime \prime}, H^{\prime}$ and $H^{\prime \prime}$ turn out to be lower semicontinuous in $H^{m}$ with respect to the first variable.

We need the following properties:

$\left(\mathrm{P}_{1}\right)$ if $\omega_{i}, A_{i} \in \mathcal{A}$, with $A_{i} \subseteq \omega_{i}(i=1,2)$ and $\omega_{1} \subseteq \omega_{2}, A_{1} \subseteq A_{2}$, then for every $u \in H^{m}$

$$
G^{\prime}\left(u, \omega_{1}, A_{1}\right) \leq G^{\prime}\left(u, \omega_{2}, A_{2}\right), \quad G^{\prime \prime}\left(u, \omega_{1}, A_{1}\right) \leq G^{\prime \prime}\left(u, \omega_{2}, A_{2}\right)
$$


$\left(\mathrm{P}_{2}\right)$ if $A, A^{\prime} \in \mathcal{A}$ and $A^{\prime} \Subset A$, then for every $u \in\left(H^{m}\right)^{+}$

$$
G^{\prime}\left(u, \mathbb{R}^{n}, A^{\prime}\right) \leq H^{\prime}(u, A, A), \quad G^{\prime \prime}\left(u, \mathbb{R}^{n}, A^{\prime}\right) \leq H^{\prime \prime}(u, A, A) .
$$

Let us prove $\left(\mathrm{P}_{1}\right)$. We consider only the first inequality, the second one being analogous.

Fix $u \in H^{m}$; by definition of $G^{\prime}\left(u, \omega_{2}, A_{2}\right)$ there exists a sequence $\left(u_{h}\right)$ in $H^{m}$ converging to $u$ in $H_{\mathrm{loc}}^{m-1}$ and such that

$$
F\left(u, \omega_{2}\right)+G^{\prime}\left(u, \omega_{2}, A_{2}\right)=\liminf _{h \rightarrow+\infty}\left[F\left(u_{h}, \omega_{2}\right)+G_{E_{\sigma(h)}}\left(u_{h}, A_{2}\right)\right] .
$$

Assume that $G^{\prime}\left(u, \omega_{2}, A_{2}\right)<+\infty$, otherwise there is nothing to prove. Then, up to a subsequence, we may suppose that $\left(u_{h}\right)$ converges to $u$ weakly in $H^{m}\left(\omega_{2}\right)$ and that the lower limit in (5.10) is actually a limit. Then for $i=1,2$

$$
\begin{aligned}
F\left(u_{h}, \omega_{i}\right)-F\left(u, \omega_{i}\right) & =F\left(u_{h}-u, \omega_{i}\right)+2 \sum_{|\alpha| \leq m} \int_{\omega_{i}} D^{\alpha}\left(u_{h}-u\right) D^{\alpha} u \mathrm{~d} x \\
& =F\left(u_{h}-u, \omega_{i}\right)+\epsilon_{h}
\end{aligned}
$$

where $\lim _{h \rightarrow+\infty} \epsilon_{h}=0$. Since $G_{E_{\sigma(h)}}\left(u_{h}, \cdot\right)$ is increasing for every $h \in \mathbb{N}$, we have

$$
\begin{aligned}
G^{\prime}\left(u, \omega_{2}, A_{2}\right) & =\lim _{h \rightarrow+\infty}\left[F\left(u_{h}-u, \omega_{2}\right)+G_{E_{\sigma(h)}}\left(u_{h}, A_{2}\right)\right] \\
& \geq \liminf _{h \rightarrow+\infty}\left[F\left(u_{h}-u, \omega_{1}\right)+G_{E_{\sigma(h)}}\left(u_{h}, A_{1}\right)\right] \\
& \geq G^{\prime}\left(u, \omega_{1}, A_{1}\right) .
\end{aligned}
$$

Let us prove $\left(\mathrm{P}_{2}\right)$. We consider only the first inequality, the second one being analogous.

Let $u \in\left(H^{m}\right)^{+}$. As in the proof of $\left(\mathrm{P}_{1}\right)$, we may suppose that $H^{\prime}(u, A, A)<+\infty$ and that there exists a sequence $\left(u_{h}\right)$ in $H^{m}$ converging to $u$ in $H_{\text {loc }}^{m-1}$ and weakly in $H^{m}(A)$, such that

$$
F(u, A)+H^{\prime}(u, A, A)=\lim _{h \rightarrow+\infty}\left[F\left(u_{h}, A\right)+H_{E_{\sigma(h)}}\left(u_{h}, A\right)\right] .
$$

Let us consider a set $A^{\prime \prime} \in \mathcal{A}$, with $A^{\prime} \Subset A^{\prime \prime} \Subset A$ and let $\varphi$ be a function in $C_{0}^{\infty}(A)$ with $\varphi=1$ on $A^{\prime \prime}$ and $0 \leq \varphi \leq 1$. Define $v_{h}=\varphi u_{h}+(1-\varphi) u$ for every $h \in \mathbb{N}$. Then $\left(v_{h}\right)$ is a sequence in $H^{m}$ which converges to $u$ in $H^{m-1}$. 
The finiteness of $H^{\prime}(u, A, A)$ implies the existence of an index $k \in \mathbb{N}$ such that $H_{E_{\sigma(h)}}\left(u_{h}, A\right)<+\infty$ whenever $h \geq k$, hence $\tilde{u}_{h} \geq 0(m, 2)$-q.e. on $A$. It follows that $v_{h} \in\left(H^{m}\right)^{+}$, thus

$$
G_{E_{\sigma(h)}}\left(v_{h}, A^{\prime}\right)=H_{E_{\sigma(h)}}\left(v_{h}, A^{\prime}\right)=H_{E_{\sigma(h)}}\left(u_{h}, A^{\prime}\right) \leq H_{E_{\sigma(h)}}\left(u_{h}, A\right)
$$

for every $h \geq k$. By the convergence properties of the sequence $\left(u_{h}\right)$, we easily obtain, for $h \geq k$,

$$
\begin{aligned}
F\left(v_{h}, \mathbb{R}^{n}\right) & =\sum_{|\alpha| \leq m} \int_{\mathbb{R}^{n}}\left|\varphi D^{\alpha}\left(u_{h}-u\right)+D^{\alpha} u\right|^{2} \mathrm{~d} x+\epsilon_{h} \leq \\
& \leq \sum_{|\alpha| \leq m} \int_{\mathbb{R}^{n}}\left[\varphi\left|D^{\alpha} u_{h}\right|^{2}+(1-\varphi)\left|D^{\alpha} u\right|^{2}\right] \mathrm{d} x+\epsilon_{h} \\
& \leq F\left(u_{h}, A\right)+F\left(u, \mathbb{R}^{n} \backslash A^{\prime \prime}\right)+\epsilon_{h}
\end{aligned}
$$

where $\lim _{h \rightarrow+\infty} \epsilon_{h}=0$. Then, by (5.12)

$$
\begin{aligned}
F\left(u, \mathbb{R}^{n}\right)+G^{\prime}\left(u, \mathbb{R}^{n}, A^{\prime}\right) \leq \liminf _{h \rightarrow+\infty}\left[F\left(v_{h}, \mathbb{R}^{n}\right)+G_{E_{\sigma(h)}}\left(v_{h}, A^{\prime}\right)\right] \\
\leq \liminf _{h \rightarrow+\infty}\left[F\left(u_{h}, A\right)+H_{E_{\sigma(h)}}\left(u_{h}, A\right)\right]+F\left(u, \mathbb{R}^{n} \backslash A^{\prime \prime}\right),
\end{aligned}
$$

and therefore, by (5.11) and (5.13),

$$
F\left(u, A^{\prime \prime}\right)+G^{\prime}\left(u, \mathbb{R}^{n}, A^{\prime}\right) \leq F(u, A)+H^{\prime}(u, A, A) .
$$

We conclude taking into account that $A^{\prime \prime}$ is an arbitrary open set such that $A^{\prime} \Subset A^{\prime \prime} \Subset A$.

We are now in a position to determine the form of $G$. It is clear that $G(u, A)=+\infty$ for every $A \in \mathcal{A}$ and $u \notin\left(H^{m}\right)^{+}$. On the other hand, if $u \in\left(H^{m}\right)^{+}$, then by properties $\left(\mathrm{P}_{1}\right)$ and $\left(P_{2}\right)$ we have

$$
G^{\prime}\left(u, A^{\prime}, A^{\prime}\right) \leq G^{\prime}\left(u, \mathbb{R}^{n}, A^{\prime}\right) \leq H^{\prime}(u, A, A) \leq G^{\prime}(u, A, A)
$$

whenever $A, A^{\prime} \in \mathcal{A}$ and $A^{\prime} \Subset A$ (note that $H_{E_{h}}(\cdot, A) \leq G_{E_{h}}(\cdot, A)$ for every $h \in \mathbb{N})$. Besides, $A \mapsto G^{\prime}(u, A, A)$ is increasing by $\left(\mathrm{P}_{1}\right)$, thus the set $\mathcal{R}_{4}$ of those $A \in \mathcal{A}$ such that

$$
G^{\prime}(u, A, A)=\sup \left\{G^{\prime}\left(u, A^{\prime}, A^{\prime}\right) \mid A^{\prime} \in \mathcal{A}, A^{\prime} \Subset A\right\}
$$


is rich in $\mathcal{A}$ (see property (5.8) for $M^{\prime}$ ). From (5.14), $G^{\prime}(u, A, A)=$ $H^{\prime}(u, A, A)$ for every $A \in \mathcal{R}_{4}$. This, together with (5.9) and the result of step 1 , yields that $G(u, A)=H(u, A)$ for every $A$ in the rich family of open sets $\mathcal{R}_{3}=\mathcal{R}_{1} \cap \mathcal{R}_{2} \cap \mathcal{R}_{4}$; we pass to an arbitrary $A \in \mathcal{A}$ observing that $G(u, \cdot)$ is increasing on $\mathcal{A}$ (as can be easily checked by using $\left(\mathrm{P}_{1}\right)$ ) and $G(u, A)=\sup \left\{G\left(u, A^{\prime}\right) \mid A^{\prime} \in \mathcal{A}, A^{\prime} \Subset A\right\}$ for every $A \in \mathcal{A}$. Hence step 3 is proved.

Step 4 To accomplish the proof of theorem 5.6 we have only to show that for a suitable rich family of open sets $A$ the convergence in (5.3) takes place, as well as the corresponding one for the sequence $\left(H_{E_{\sigma(h)}}\right)$.

If $u \notin\left(H^{m}\right)^{+}$then $G^{\prime}\left(u, \mathbb{R}^{n}, A\right)=G^{\prime \prime}\left(u, \mathbb{R}^{n}, A\right)=+\infty=G(u, A)$ for every $A \in \mathcal{A}$. In the case $u \in\left(H^{m}\right)^{+}$, by $\left(\mathrm{P}_{1}\right)$ and $\left(\mathrm{P}_{2}\right)$ we have, as in (5.14),

$$
G^{\prime}\left(u, A^{\prime}, A^{\prime}\right) \leq G^{\prime}\left(u, \mathbb{R}^{n}, A^{\prime}\right) \leq G^{\prime}(u, A, A) .
$$

We can now use an argument quite similar to the one applied in the previous step to (5.14). Since the same holds for $G^{\prime \prime}$, we obtain that (5.3) is valid for a rich family of open sets $A$. Finally, as to $\left(H_{E_{\sigma(h)}}\right)$, we argue in the same way, taking into account that for every $u \in H^{m}$ and $A, A^{\prime} \in \mathcal{A}$ with $A^{\prime} \Subset A$ we have

$$
H^{\prime}\left(u, \mathbb{R}^{n}, A^{\prime}\right) \leq H^{\prime}(u, A, A), \quad H^{\prime \prime}\left(u, \mathbb{R}^{n}, A^{\prime}\right) \leq H^{\prime \prime}(u, A, A) .
$$

This replaces $\left(\mathrm{P}_{2}\right)$ and can be proved in a similar (and even simpler) way.

Now we represent the $\Gamma$-limit of the foregoing theorem by means of an integral functional.

By a Borel measure on $\mathbb{R}^{n}$ we mean a non-negative countably additive set function $\mu: \mathcal{B} \rightarrow[0,+\infty]$ such that $\mu(\emptyset)=0$. We indicate by $\mathcal{M}_{m, 2}$ the class of all Borel measures on $\mathbb{R}^{n}$ such that $\mu(B)=0$ for every $B \in \mathcal{B}$ with null $(m, 2)$-capacity. If $\mu$ is a measure in $\mathcal{M}_{m, 2}$, we still denote by $\mu$ its completion, which is defined, in particular, on all $(m, 2)$-quasi Borel sets.

Lemma 5.7. - Let $\left(E_{h}\right)$ be a sequence of subsets of $\mathbb{R}^{n}$. Then there exist a subsequence $\left(E_{\sigma(h)}\right)$ of $\left(E_{h}\right)$, a measure $\mu \in \mathcal{M}_{m, 2}$ and a set $\mathcal{R}^{\prime} \subseteq \mathcal{A}$, rich in $\mathcal{A}$, such that for every $A \in \mathcal{R}^{\prime}$

$\left[F\left(\cdot, \mathbb{R}^{n}\right)+G_{E_{\sigma(h)}}(\cdot, A)\right] \quad \Gamma\left(H_{\text {loc }}^{m-1}\right)$-converges to $\left[F\left(\cdot, \mathbb{R}^{n}\right)+G(\cdot, A)\right]$, 
where

$$
G(u, A)= \begin{cases}\int_{A}\left[(1-\tilde{u})^{+}\right]^{2} \mathrm{~d} \mu & \text { if } u \in\left(H^{m}\right)^{+} \\ +\infty & \text { otherwise in } H^{m}\end{cases}
$$

for every $u \in H^{m}$ and $A \in \mathcal{A}$.

Proof. - Let us apply theorem 5.6 and let $\left(E_{\sigma(h)}\right), G, \mathcal{R}^{\prime}$ and $H$ as in that statement. The only step which remains is to determine the form of $H$. Properties (i) to (iv) satisfied by $H$ allows us to represent it by means of theorem 5.7 in [8]. Then there exist a Borel function $f: \mathbb{R}^{n} \times \mathbb{R} \rightarrow[0,+\infty]$ and two non-negative Borel measures $\lambda$ and $\nu$ on $\mathbb{R}^{n}$ such that

$$
H(u, A)=\int_{A} f(x, \tilde{u}(x)) \mathrm{d} \lambda(x)+\nu(A)
$$

for every $u \in H^{m}$ and every $A \in \mathcal{A}$. Furthermore, the same theorem guarantees that $\lambda$ is a Radon measure which belongs to $H^{-m}$, hence to $\mathcal{M}_{m, 2}$, and that for every $x \in \mathbb{R}^{n}$ the function $f(x, \cdot)$ is decreasing and lower semicontinuous on $\mathbb{R}$.

If $A$ is an arbitrary bounded open set of $\mathcal{R}^{\prime}$, and $\varphi$ a function in $C_{0}^{\infty}\left(\mathbb{R}^{n}\right)$ with $\varphi=1$ on $A$, then $H(\varphi, A)=0$ by theorem 5.6. We infer that $\nu \equiv 0$ and $f(\cdot, 1)=0 \lambda$-a.e. on $\mathbb{R}^{n}$. Moreover, since $\Gamma$-convergence is stable under continuous perturbations (see, for example, theorem 2.15 in [3]), from theorem 5.6 we obtain, for every $u \in H^{m}$,

$$
\begin{aligned}
\Gamma\left(H_{\mathrm{loc}}^{m-1}\right)-\lim _{\substack{h \rightarrow+\infty \\
v \rightarrow u}}\left[\sum_{0<|\alpha| \leq m} \int_{A}\left|D^{\alpha} v\right|^{2} \mathrm{~d} x+H_{E_{\sigma(h)}}(v, A)\right]= \\
=\sum_{0<|\alpha| \leq m} \int_{A}\left|D^{\alpha} u\right|^{2} \mathrm{~d} x+H(u, A),
\end{aligned}
$$

where $A$ is as above. Observe now that for every $h \in \mathbb{N}$

$$
H_{E_{h}}(t u+(1-t) \varphi, A)=t^{2} H_{E_{h}}(u, A)
$$

whenever $t \in \mathbb{R}^{+}$and $u \in H^{m}$ are such that $u \geq 0$ a.e. on $A$ and $t u+(1-t) \varphi \geq 0$ a.e. on $A$. This turns out to be a positive-homogeneity property for the functional $\widetilde{H}_{E_{h}}(\cdot, A)=H_{E_{h}}(\varphi-\cdot, A)$ for every $t \in \mathbb{R}^{+}$ and $v \in H^{m}$ with $v \leq 1$ and $t v \leq 1$ a.e. on $A$. As this property is preserved by $\Gamma$-convergence (see, for instance, theorem 10.9 in [10]), on account of (5.17) it also holds for the functional $H$ and for the fixed $A$. By the 
richness of $\mathcal{R}^{\prime}$ this is true for every $A \in \mathcal{A}$. We are now in a position to apply the next lemma, which proves that

$$
\int_{A} f(x, \tilde{u}) \mathrm{d} \lambda=\int_{A} f(x, 0)\left[(1-\tilde{u})^{+}\right]^{2} \mathrm{~d} \lambda
$$

for every $A \in \mathcal{A}$ and $u \in H^{m}$ with $u \geq 0$ a.e. on $A$. We conclude setting $\mathrm{d} \mu=f(\cdot, 0) \mathrm{d} \lambda$.

Lemma 5.8. - Let $\lambda \in \mathcal{M}_{m, 2}$ and let $g: \mathbb{R}^{n} \times \mathbb{R} \rightarrow[0,+\infty]$ be a Borel function such that

(i) for every $x \in \mathbb{R}^{n}$, the function $g(x, \cdot)$ is increasing and lower semicontinuous on $\mathbb{R}$;

(ii) $g(x, 0)=0$ for $\lambda$-a.e. $x \in \mathbb{R}^{n}$;

(iii) for every $A \in \mathcal{A}$ and $u \in H^{m}$ with $u \leq 1$ a.e., we have

$$
\int_{A} g(x, t \tilde{u}) \mathrm{d} \lambda=t^{2} \int_{A} g(x, \tilde{u}) \mathrm{d} \lambda
$$

whenever $t \in \mathbb{R}^{+}$is such that $t u \leq 1$ a.e.

Then

$$
\int_{A} g(x, \tilde{u}) \mathrm{d} \lambda=\int_{A} g(x, 1)\left(\tilde{u}^{+}\right)^{2} \mathrm{~d} \lambda
$$

for every $A \in \mathcal{A}$ and $u \in H^{m}(A)$ with $u \leq 1$ a.e. on $A$.

Proof. - The result is standard if $\int_{\mathbb{R}^{n}} g(x, \tilde{u}) \mathrm{d} \lambda<+\infty$ for every $u \in H^{m}$. In the general case we shall essentially follow the proof of lemma 2.3 in [12].

Let us consider the set

$$
S=\left\{u \in H^{m} \mid \int_{\mathbb{R}^{n}} g(x, \tilde{u}) \mathrm{d} \lambda<+\infty\right\} .
$$

Since $H^{m}$ is a separable metric space, there exists a sequence $\left(u_{h}\right)$ in $S$ which is dense in $S$ with respect to the strong topology of $H^{m}$. For every $h \in \mathbb{N}$ we consider an $(m, 2)$-q.e. representative of $\tilde{u}_{h}$ and we shall keep it fixed in the following arguments. Define $E=\bigcup_{h, k \in \mathbb{N}}\left\{\tilde{u}_{h} \geq 1 / k\right\}$. We now prove that

$$
g(x, t)=g(x, 1)\left(t^{+}\right)^{2}
$$

for $\lambda$-a.e. $x \in E$ and for every $t \in \mathbb{R}$ with $t \leq 1$. 
Let $h, k \in \mathbb{N}$; since the characteristic function of $\left\{\tilde{u}_{h} \geq 1 / k\right\}$ is quasi lower semicontinuous, by lemma 2.1 we can find a sequence $\left(v_{h, k ; i}\right)_{i}$ in $H^{m}$ such that $\left(\tilde{v}_{h, k ; i}\right)_{i}$ is increasing $(m, 2)$-q.e. and

$$
\tilde{v}_{h, k ; i} \rightarrow \mathbf{1}_{\left\{\tilde{u}_{h}>1 / k\right\}} \quad(m, 2) \text {-q.e. as } i \rightarrow+\infty .
$$

For every $i \in \mathbb{N}$, we fix an $(m, 2)$-q.e. representative of $\tilde{v}_{h, k ; i}$, as we have done for the sequence $\left(u_{h}\right)$. Denote by $N_{1}$ the $(m, 2)$-negligible set of the points where $\left(\tilde{v}_{h, k ; i}\right)_{i}$ fails to be increasing or to satisfy $(5.20)$ for some $h, k \in \mathbb{N}$.

For every $x \in \mathbb{R}^{n}$ the function $g(x, \cdot)$ is increasing on $\mathbb{R}$ and, by (ii), $g(x, \eta)=0$ for every $\eta \leq 0$ and $x \in \mathbb{R}^{n} \backslash N_{2}$, where $\lambda\left(N_{2}\right)=0$; then we have

$$
\begin{aligned}
\int_{\mathbb{R}^{n}} g\left(x, \frac{1}{k} \tilde{v}_{h, k ; i}\right) \mathrm{d} \lambda & \leq \int_{\mathbb{R}^{n}} g\left(x, \frac{1}{k} \mathbf{1}_{\left\{\tilde{u}_{h}>1 / k\right\}}\right) \mathrm{d} \lambda \\
& \leq \int_{\mathbb{R}^{n}} g\left(x, \tilde{u}_{h}\right) \mathrm{d} \lambda<+\infty ;
\end{aligned}
$$

hence, by (iii),

$$
\int_{\mathbb{R}^{n}} g\left(x, \tilde{v}_{h, k ; i}\right) \mathrm{d} \lambda<+\infty
$$

We apply now the classical result. For every $\tau \in[0,1], h, k, i \in \mathbb{N}$ and $A \in \mathcal{A}$, from (iii) we get

$$
\int_{A} g\left(x, \tau \tilde{v}_{h, k ; i}(x)\right) \mathrm{d} \lambda=\tau^{2} \int_{A} g\left(x, \tilde{v}_{h, k ; i}(x)\right) \mathrm{d} \lambda<+\infty ;
$$

therefore there exists a Borel set $N_{3}$ such that $\lambda\left(N_{3}\right)=0$ and

$$
g\left(x, \tau \tilde{v}_{h, k ; i}(x)\right)=\tau^{2} g\left(x, \tilde{v}_{h, k ; i}(x)\right)
$$

for every $x \in \mathbb{R}^{n} \backslash N_{3}, h, k, i \in \mathbb{N}$ and $\tau \in \mathbb{Q} \cap[0,1]$. This equality actually holds whenever $\tau \in[0,1]$, since by (i) the function $g(x, \cdot)$ is continuous from the left for every $x \in \mathbb{R}^{n}$. Let $x \in E \backslash\left(N_{1} \cup N_{3}\right)$ and $t_{0} \in \mathbb{R}$ with $0<t_{0}<1$. By definition of $E$ there exist $h, k \in \mathbb{N}$ such that $\tilde{u}_{h}(x)>1 / k$; then (5.20) yields that $\tilde{v}_{h, k ; i}(x)>t_{0}$ for a suitable index $i \in \mathbb{N}$. For every $t \in\left[0, t_{0}\right]$ we can apply (5.21) with $\tau=t / \tilde{v}_{h, k ; i}(x)$, so that

$$
g(x, t)=t^{2} \frac{g\left(x, \tilde{v}_{h, k ; i}(x)\right)}{\left(\tilde{v}_{h, k ; i}(x)\right)^{2}} .
$$


Let $t_{1}, t_{2} \in \mathbb{R}$ with $0<t_{1}<t_{2}<1$ and take $t_{2}$ as $t_{0}$ in the above argument. Since in (5.22) the choice of the indexes $h, k$ and $i$ does not depend on $t$, we have

$$
\frac{g\left(x, t_{1}\right)}{t_{1}^{2}}=\frac{g\left(x, t_{2}\right)}{t_{2}^{2}}
$$

by continuity from the left this inequality extends to $t_{2}=1$. We conclude that $g(x, t)=g(x, 1) t^{2}$ for every $x \in E \backslash\left(N_{1} \cup N_{3}\right)$ and every $t \in \mathbb{R}$ with $0<t \leq 1$. Hence (5.19) follows for every $x \in E \backslash\left(N_{1} \cup N_{2} \cup N_{3}\right)$ and $t \leq 1$.

Now we claim that

$$
\int_{\mathbb{R}^{n}} g(x, \tilde{u}) \mathrm{d} \lambda=\int_{\mathbb{R}^{n}} g(x, 1)\left(\tilde{u}^{+}\right)^{2} \mathrm{~d} \lambda
$$

for every $u \in H^{m}$ with $u \leq 1$ a.e.

Assume that the left hand side of (5.23) is finite. By the density of $\left(u_{h}\right)$ in $S$ we obtain that $\{\tilde{u}>0\}$ is quasi contained in $E$. Then, from (5.19)

$$
g(x, \tilde{u}(x))=g(x, 1)(\tilde{u}(x))^{2} \text { for } \lambda \text {-a.e. } x \in\{\tilde{u}>0\} ;
$$

this immediately implies that $u$ satisfies (5.23).

At this point, to accomplish the proof of (5.23) for every $u \in H^{m}$ with $u \leq 1$ a.e., it only remains to consider the case in which the right hand side of (5.23) is finite. Let $\epsilon>0$; as before we can find a sequence $\left(v_{h}\right)$ in $H^{m}$ such that $\left(\tilde{v}_{h}\right)$ increases and converges $(m, 2)$-q.e. to $\mathbf{1}_{\{\tilde{u}>\epsilon\}}$. Therefore, for every $h \in \mathbb{N}$

$$
\begin{aligned}
\int_{\mathbb{R}^{n}} g\left(x, \tilde{v}_{h}\right) \mathrm{d} \lambda & \leq \int_{\mathbb{R}^{n}} g\left(x, \mathbf{1}_{\{\tilde{u}>\epsilon\}}\right) \mathrm{d} \lambda \\
& \leq \frac{1}{\epsilon^{2}} \int_{\mathbb{R}^{n}} g(x, 1)\left(\tilde{u}^{+}\right)^{2} \mathrm{~d} \lambda<+\infty .
\end{aligned}
$$

Hence $v_{h} \in S$ for every $h \in \mathbb{N}$. This implies that $\left\{\tilde{v}_{h}>0\right\}$ is quasi contained in $E$, so that, by the arbitrary choice of $\epsilon>0$, the set $\{\tilde{u}>0\}$ is quasi contained in $E$. Finally, we get that $u$ satisfies (5.23) by applying once more equality (5.19).

We are now in a position to prove (5.18). Let $A \in \mathcal{A}$ and $u \in H^{m}(A)$ with $u \leq 1$ a.e. on $A$. For every open set $A^{\prime} \Subset A$ let $\varphi \in C_{0}^{\infty}(A)$ with $\varphi=1$ on $A^{\prime}$ and $0 \leq \varphi \leq 1$. Then, by (5.23)

$$
\begin{aligned}
\int_{A^{\prime}} g(x, \tilde{u}) \mathrm{d} \lambda & \leq \int_{\mathbb{R}^{n}} g(x, \varphi \tilde{u}) \mathrm{d} \lambda \\
& \leq \int_{\mathbb{R}^{n}} g(x, 1)\left[(\varphi \tilde{u})^{+}\right]^{2} \mathrm{~d} \lambda \leq \int_{A} g(x, 1)\left(\tilde{u}^{+}\right)^{2} \mathrm{~d} \lambda . \\
-138- &
\end{aligned}
$$


As $A^{\prime}$ is an arbitrary open set satisfying $A^{\prime} \Subset A$, we get

$$
\int_{A} g(x, \tilde{u}) \mathrm{d} \lambda \leq \int_{A} g(x, 1)\left(\tilde{u}^{+}\right)^{2} \mathrm{~d} \lambda .
$$

The opposite inequality, which can be obtained in a similar way, concludes the proof of the lemma.

It is now convenient to introduce an auxiliary notion of capacity (see [11]).

Let $\nu \in \mathcal{M}_{m, 2}$. For every $(m, 2)$-quasi Borel subset $B$ of $\mathbb{R}^{n}$, define

$$
\begin{aligned}
\operatorname{cap}_{\nu}(B) & =\inf _{u \in\left(H^{m}\right)^{+}}\left[\|u\|_{m, 2}^{2}+\int_{B}\left[(1-\tilde{u})^{+}\right]^{2} \mathrm{~d} \nu\right] \\
& =\inf _{u \in\left(H^{m}\right)^{+}}\left[F\left(u, \mathbb{R}^{n}\right)+G_{\nu}(u, B)\right],
\end{aligned}
$$

where we have put

$$
G_{\nu}(u, B)=\int_{B}\left[(1-\tilde{u})^{+}\right]^{2} \mathrm{~d} \nu
$$

for every $u \in H^{m}$. Note that the functional $\left[F\left(\cdot, \mathbb{R}^{n}\right)+G_{\nu}(\cdot, B)\right]$ is lower semicontinuous in the strong topology of $H^{m}$ (use proposition 1.3) and (strictly) convex, hence it is weakly lower semicontinuous in $H^{m}$. Then the infimum in (5.24) is attained.

We shall need the following properties of $\operatorname{cap}_{\nu}$ (see theorem 2.9 in [11]).

Proposition 5.9. - Let $\nu \in \mathcal{M}_{m, 2}$. Then:

(i) if $\left(B_{h}\right)$ is an increasing sequence of $(m, 2)$-quasi Borel sets and $B=\bigcup_{h} B_{h}$, then $\operatorname{cap}_{\nu}(B)=\sup _{h} \operatorname{cap}_{\nu}\left(B_{h}\right)$;

(ii) $\operatorname{cap}_{\nu}(B) \leq C_{m, 2}(B)$ for every $(m, 2)$-quasi Borel set $B$;

(iii) for every $(m, 2)$-quasi open set $A$

$$
\operatorname{cap}_{\nu}(A)=\inf \left\{\operatorname{cap}_{\nu}(U) \mid U \text { open, } U \supseteq A\right\}
$$

\section{Proof}

(i) Since $\operatorname{cap}_{\nu}$ is obviously increasing, it suffices to prove that $\operatorname{cap}_{\nu}(B) \leq$ $\sup _{h} \operatorname{cap}_{\nu}\left(B_{h}\right)$ assuming that the right hand side is finite. For every $h \in \mathbb{N}$, let $w_{h} \in\left(H^{m}\right)^{+}$be the unique solution of the minimum problem defining 
$\operatorname{cap}_{\nu}\left(B_{h}\right)$; the sequence $\left(w_{h}\right)$ is bounded in $H^{m}$, hence there exists a subsequence, still denoted by $\left(w_{h}\right)$, which converges weakly in $H^{m}$ to a function $w \in\left(H^{m}\right)^{+}$. By weak lower semicontinuity we have, for every $k \in \mathbb{N}$,

$$
\begin{aligned}
F\left(w, \mathbb{R}^{n}\right)+G_{\nu}\left(w, B_{k}\right) & \leq \liminf _{h \rightarrow+\infty}\left[F\left(w_{h}, \mathbb{R}^{n}\right)+G_{\nu}\left(w_{h}, B_{k}\right)\right] \\
& \leq \liminf _{h \rightarrow+\infty}\left[F\left(w_{h}, \mathbb{R}^{n}\right)+G_{\nu}\left(w_{h}, B_{h}\right)\right]= \\
& =\sup _{h} \operatorname{cap}_{\nu}\left(B_{h}\right) .
\end{aligned}
$$

We conclude taking the limit as $k \rightarrow+\infty$.

(ii) It suffices to assume $C_{m, 2}(B)<+\infty$ and use, as a test function in (5.24), the element $w$ of $\left(H^{m}\right)^{+}$such that $\|w\|_{m, 2}^{2}=C_{m, 2}(B)$ and $\tilde{w} \geq 1$ $(m, 2)$-q.e. on $B$.

(iii) Let $A$ be an $(m, 2)$-quasi open set with $\operatorname{cap}_{\nu}(A)<+\infty$ (otherwise there is nothing to prove). For every $\epsilon>0$ there exists an open set $\omega$ such that $C_{m, 2}(\omega)<\epsilon$ and $A \cup \omega$ is open; let $w_{1}$ and $w_{2}$ be the solutions of the minimum problems defining $\operatorname{cap}_{\nu}(A)$ and $\operatorname{cap}_{\nu}(\omega)$, respectively. On account of the fact that $w_{1}$ and $w_{2}$ are non-negative functions, for every $0<\sigma<1$, we have

$$
\begin{aligned}
\operatorname{cap}_{\nu}(A \cup \omega) & \leq\left\|w_{1}+w_{2}\right\|_{m, 2}^{2}+\int_{A \cup \omega}\left[\left(1-\left(\tilde{w}_{1}+\tilde{w}_{2}\right)\right)^{+}\right]^{2} \mathrm{~d} \nu \\
& \leq \frac{1}{1-\sigma} \operatorname{cap}_{\nu}(A)+\frac{1}{\sigma} \operatorname{cap}_{\nu}(\omega)
\end{aligned}
$$

and since, by (ii), $\operatorname{cap}_{\nu}(\omega) \leq C_{m, 2}(\omega)$, it follows

$$
\operatorname{cap}_{\nu}(A \cup \omega)-\operatorname{cap}_{\nu}(A) \leq \frac{\sigma}{1-\sigma} \operatorname{cap}_{\nu}(A)+\frac{\epsilon}{\sigma} .
$$

Choosing $\sigma=\epsilon^{1 / 2}$, the right hand side tends to zero with $\epsilon$. This concludes the proof.

For every subset $E$ of $\mathbb{R}^{n}$, define

$$
\mu_{E}^{\infty}(B)= \begin{cases}0 & \text { if } C_{m, 2}(E \cap B)=0 \\ +\infty & \text { if } C_{m, 2}(E \cap B)>0\end{cases}
$$

for every $B \subseteq \mathbb{R}^{n}$. It is clear that $\mu_{E}^{\infty} \in \mathcal{M}_{m, 2}$. 
In the following lemma we shall use the condition of convergence of capacities stated in theorem 5.1.

LEMMA 5.10. - Let $\left(E_{h}\right)$ be a sequence of subsets of $\Omega$ and $E$ be a subset of $\Omega$. Assume the existence of a set $\mathcal{R} \subseteq \mathcal{A}(\Omega)$, rich in $\mathcal{A}(\Omega)$, such that

$$
\lim _{h \rightarrow+\infty} C_{m, 2}\left(E_{h} \cap A\right)=C_{m, 2}(E \cap A)
$$

for every $A \in \mathcal{R}$. Assume, in addition, that $E$ is $(m, 2)$-quasi closed. If $\left(E_{\sigma(h)}\right), \mu$ and $\mathcal{R}^{\prime}$ are as in lemma 5.7, then $\mu(A)=\mu_{E}^{\infty}(A)$ for every $(m, 2)$-quasi open set $A \subseteq \Omega$.

Proof. - We prove first that for every $(m, 2)$-quasi open set $A \subseteq \Omega$

$$
\min _{u \in\left(H^{m}\right)^{+}}\left[F\left(u, \mathbb{R}^{n}\right)+G_{\mu}(u, A)\right]=C_{m, 2}(E \cap A) .
$$

Let us consider a bounded open set $A$ in $\mathcal{R}^{\prime \prime}=\mathcal{R} \cap \mathcal{R}^{\prime}$, which is a rich set in $\mathcal{A}(\Omega)$. By the boundedness of the sequence $\left(C_{m, 2}\left(E_{\sigma(h)} \cap A\right)\right)$ and recalling the definition of $G_{E_{\sigma(h)}}$, we can apply theorem 5.4 to the sequence of functionals $\left[F\left(\cdot, \mathbb{R}^{n}\right)+G_{E_{\sigma(h)}}(\cdot, A)\right]$, which $\Gamma\left(H_{\text {loc }}^{m-1}\right)$-converges to $\left[F\left(\cdot, \mathbb{R}^{n}\right)+G(\cdot, A)\right]$ since $(5.15)$ holds. Therefore, in view of $(5.25)$, we have

$$
\min _{u \in H^{m}}\left[F\left(u, \mathbb{R}^{n}\right)+G(u, A)\right]=C_{m, 2}(E \cap A) .
$$

By (5.16) this implies the validity of (5.26) for every bounded $A \in \mathcal{R}^{\prime \prime}$. Observe now that $(5.26)$ can be written as

$$
\operatorname{cap}_{\mu}(A)=\operatorname{cap}_{\mu_{E}^{\infty}}(A) .
$$

Proposition 5.9 (i) and the richness of $\mathcal{R}^{\prime \prime}$ in $\mathcal{A}(\Omega)$ yields that $\left(5.26^{\prime}\right)$ holds for every $A \in \mathcal{A}(\Omega)$; finally, we pass to any $(m, 2)$-quasi open set $A \subseteq \Omega$ by means of proposition 5.9 (iii).

Now let us take, in (5.26), $A=\Omega \backslash E$, which is quasi open because $E$ is quasi closed. If $w$ is the minimum point of $\left[F\left(\cdot, \mathbb{R}^{n}\right)+G_{\mu}(\cdot, A)\right]$ in $\left(H^{m}\right)^{+}$, then $w \equiv 0$ and $G_{\mu}(0, A)=G_{\mu}(w, A)=0$. Consequently, $\mu(\Omega \backslash E)=0$.

To accomplish the proof of the lemma it is enough to show that for every $(m, 2)$-quasi open and bounded set $A \subseteq \Omega$ we have

$$
\mu(A)=+\infty \text { if } C_{m, 2}(E \cap A)>0 .
$$


Assume $\mu(A)<+\infty$ and let $w \in\left(H^{m}\right)^{+}$be such that $\|w\|_{m, 2}^{2}=$ $C_{m, 2}(E \cap A)$ and $\tilde{w} \geq 1(m, 2)$-q.e. on $E \cap A$. Since $\mu(A \backslash E)=0$, we obtain $G_{\mu}(w, A)=0$ and, by $(5.26), w$ turns out to minimize $\left[F\left(\cdot, \mathbb{R}^{n}\right)+G_{\mu}(\cdot, A)\right]$ in $\left(H^{m}\right)^{+}$. It follows that for every $0<\epsilon<1$

$$
F\left((1-\epsilon) w, \mathbb{R}^{n}\right)+G_{\mu}((1-\epsilon) w, A)-F\left(w, \mathbb{R}^{n}\right) \geq 0,
$$

hence, taking into account that $\mu(A \backslash E)=0$ and $\tilde{w} \geq 1(m, 2)$-q.e. on $E \cap A$, we obtain

$$
-2 \epsilon\|w\|_{m, 2}^{2}+\epsilon^{2}\left[\|w\|_{m, 2}^{2}+\mu(A)\right] \geq 0 .
$$

As this inequality holds for every $0<\epsilon<1$, we must have $\|w\|_{m, 2}=0$, i.e. $C_{m, 2}(E \cap A)=0$. Thus (5.27) is proved.

Lemma 5.11. - Let $E$ be a subset of $\mathbb{R}^{n}, \widetilde{E}$ its ( $\left.m, 2\right)$-quasi closure and $A$ an $(m, 2)$-quasi open set. Then the $(m, 2)$-quasi closure of $\bar{E} \cap A$ coincides with the ( $m, 2)$-quasi closure of $E \cap A$ up to sets of zero $(m, 2)$-capacity. Consequently, $\tilde{E} \cap A$ and $E \cap A$ have the same $(m, 2)$-capacity.

Proof. - Let $F$ and $F^{\prime}$ be the quasi closures of $E \cap A$ and $\widetilde{E} \cap A$, respectively. Since $E \cap A$ is quasi contained in $\widetilde{E} \cap A, F$ is quasi contained in $F^{\prime}$. Let us prove the opposite inclusion. Let $G$ be a quasi open set such that $\operatorname{cap}(G \cap(\widetilde{E} \cap A))>0$; then $G \cap A$ is quasi open and $\operatorname{cap}((G \cap A) \cap \widetilde{E})=\operatorname{cap}(G \cap(\tilde{E} \cap A))>0$. By proposition 1.5 for $Z=\tilde{E}$, we have $\operatorname{cap}((G \cap A) \cap E)>0$. Hence, $\operatorname{cap}(G \cap(E \cap A))>0$ for every quasi open set $G$ such that $\operatorname{cap}(G \cap(\widetilde{E} \cap A))>0$. Apply now proposition 1.5 for $Z=\widetilde{E} \cap A$; then $\widetilde{E} \cap A$ is quasi contained in the quasi closure of $E \cap A$. It follows that $F^{\prime}$ is quasi contained in $F$. We conclude that $F$ is $(m, 2)$-equivalent to $F^{\prime}$.

Finally, the quasi stability of the capacity (see remark 2.11) yields

$$
\operatorname{cap}(\tilde{E} \cap A)=\operatorname{cap}\left(F^{\prime}\right)=\operatorname{cap}(F)=\operatorname{cap}(E \cap A) .
$$

Proof of theorem 5.1.- Assume that

$$
K_{0}\left(\mathbf{1}_{E_{h}}\right) \rightarrow K_{0}\left(\mathbf{1}_{E}\right) \quad \text { in } H_{0}^{m}(\Omega) .
$$

By theorem 3.1 this implies the convergence of $\left(K_{0}\left(\chi_{E_{h}}+1\right)\right)$ to $K_{0}\left(\chi_{E}+1\right)$. 
Therefore, by means of proposition 4.2 , we get the existence of a set $\mathcal{R} \subseteq$ $\mathcal{A}(\Omega)$, rich in $\mathcal{A}(\Omega)$, such that $\left(K_{0}\left(\chi_{E_{h} \cap A}+1\right)\right)$ converges to $K_{0}\left(\chi_{E \cap A}+1\right)$ for every $A \in \mathcal{R}$. Again from theorem 3.1 it follows that

$$
K_{0}\left(\mathbf{1}_{E_{h} \cap A}\right) \rightarrow K_{0}\left(\mathbf{1}_{E \cap A}\right) \text { in } H_{0}^{m}(\Omega)
$$

for every $A \in \mathcal{R}$. In view of lemma 3.2

$$
K\left(\mathbf{1}_{E_{h} \cap A}\right) \rightarrow K\left(\mathbf{1}_{E \cap A}\right) \text { in } H^{m} .
$$

Therefore, proposition 1.9 yields (5.1) for every $A \in \mathcal{R}$.

Conversely, assume that there exists a set $\mathcal{R} \subseteq \mathcal{A}(\Omega)$, rich in $\mathcal{A}(\Omega)$, such that (5.1) holds for every $A \in \mathcal{R}$. Observe now that $K_{0}\left(\mathbf{1}_{E}\right)=K_{0}\left(\mathbf{1}_{\tilde{E}}\right)$ (see remark 2.11) and that, by lemma 5.11, $C_{m, 2}(E \cap A)=C_{m, 2}(\tilde{E} \cap A)$ for every open set $A$. Consequently, since $\widetilde{E}$ is $(m, 2)$-quasi contained in $\Omega$, we may suppose that $E$ is $(m, 2)$-quasi closed.

By lemmas 5.7 and 5.10 , every subsequence $\left(E_{\sigma(h)}\right)$ of $\left(E_{h}\right)$ contains a further subsequence, still denote by $\left(E_{\sigma(h)}\right)$, for which there exist a measure $\mu_{\sigma} \in \mathcal{M}_{m, 2}$ and a set $\mathcal{R}_{\sigma} \subseteq \mathcal{A}(\Omega)$, rich in $\mathcal{A}(\Omega)$, such that for every $A \in \mathcal{R}_{\sigma}$ $\left[F\left(\cdot, \mathbb{R}^{n}\right)+G_{E_{\sigma(h)}}(\cdot, A)\right] \quad \Gamma\left(H_{\text {loc }}^{m-1}\right)$-converges to $\left[F\left(\cdot, \mathbb{R}^{n}\right)+G(\cdot, A)\right]$, where

$$
G(u, A)= \begin{cases}\int_{A}\left[(1-\tilde{u})^{+}\right]^{2} \mathrm{~d} \mu_{\sigma} & \text { if } u \in\left(H^{m}\right)^{+} \\ +\infty & \text { otherwise in } H^{m}\end{cases}
$$

for every $u \in H^{m}$ and $A \in \mathcal{A}$. Moreover, if $A \subseteq \Omega$ is quasi open, then $\mu_{\sigma}(A)=\mu_{E}^{\infty}(A)$. It is now easy to verify that $G(u, A)=G_{E}(u, A)$ for every $u \in H^{m}$ and $A \in \mathcal{A}$. Indeed, for every $0<\epsilon<1$ the set $\{\tilde{u}<1-\epsilon\} \cap A$ is quasi open and

$$
\int_{A}\left[(1-\tilde{u})^{+}\right]^{2} \mathrm{~d} \mu_{\sigma} \geq \epsilon^{2} \mu_{\sigma}(\{\tilde{u}<1-\epsilon\} \cap E \cap A) .
$$

So far we have proved that the $\Gamma$-limit does not depend on the chosen subsequence. In order to obtain that the whole sequence converges, it is enough to apply proposition 15.5 and 15.7 (Urysohn property of $\Gamma$ convergence) in [10] (see also propositions 4.11 and 4.14 in [13]). Therefore, we obtain the existence of a set $\mathcal{R}_{0} \subseteq \mathcal{A}(\Omega)$, rich in $\mathcal{A}(\Omega)$, such that

$$
\left[F\left(\cdot, \mathbb{R}^{n}\right)+G_{E_{h}}(\cdot, A)\right] \quad \Gamma\left(H_{\text {loc }}^{m-1}\right) \text {-converges to }\left[F\left(\cdot, \mathbb{R}^{n}\right)+G_{E}(\cdot, A)\right]
$$


for every $A \in \mathcal{R}_{0}$. From proposition 5.5 it follows that

$$
K\left(\mathbf{1}_{E_{h} \cap A}\right) \rightarrow K\left(\mathbf{1}_{E \cap A}\right) \quad \text { in } H^{m}
$$

hence by lemma 3.2 ,

$$
K_{0}\left(\mathbf{1}_{E_{h} \cap A}\right) \rightarrow K_{0}\left(\mathbf{1}_{E \cap A}\right) \quad \text { in } H_{0}^{m}(\Omega)
$$

for every $A \in \mathcal{R}_{0}$. Choose $A \in \mathcal{R}_{0}$ with the property $\Omega^{\prime} \Subset A \Subset \Omega$; since the sets $E_{h}$ and $E$ are contained in $\Omega^{\prime}$, we conclude that

$$
K_{0}\left(\mathbf{1}_{E_{h}}\right) \rightarrow K_{0}\left(\mathbf{1}_{E}\right) \text { in } H_{0}^{m}(\Omega) .
$$

This completes the proof of the theorem.

Theorem 5.12. - Let $\left(\psi_{h}\right)$ be a sequence of functions from $\Omega$ into $\overline{\mathbb{R}}$ and let $\psi$ be a function from $\Omega$ into $\overline{\mathbb{R}}$. Then

$$
K_{0}\left(\psi_{h}\right) \rightarrow K_{0}(\psi) \text { in } H_{0}^{m}(\Omega) \quad \text { and } \quad K_{0}(\psi) \neq \emptyset
$$

if and only if the following conditions (a) and (b) are satisfied:

(a) there exist a dense set $D$ in $\mathbb{R}$ and a set $\mathcal{R} \subseteq \mathcal{A}(\Omega)$, rich in $\mathcal{A}(\Omega)$, such that

$$
\lim _{h \rightarrow+\infty} C_{m, 2}\left(\left\{\psi_{h}>t\right\} \cap A\right)=C_{m, 2}(\{\psi>t\} \cap A)
$$

for every $t \in D$ and $A \in \mathcal{R}$;

(b) there exist a sequence $\left(w_{h}\right)$ in $H_{0}^{m}(\Omega)$ and an index $k \in \mathbb{N}$ such that $\left(w_{h}\right)$ converges strongly in $H_{0}^{m}(\Omega)$ and $w_{h} \in K_{0}\left(\psi_{h}\right)$ for every $h \geq k$.

For the proof it is convenient to recall a fact about the rich sets introduced in section 1. Let $\alpha: \mathcal{A}(\Omega) \rightarrow \overline{\mathbb{R}}$ be an increasing function, i.e. $\alpha(A) \leq \alpha(B)$ whenever $A, B \in \mathcal{A}(\Omega)$ and $A \subseteq B$. Let $\alpha_{-}$and $\alpha_{+}$be the functions on $\mathcal{A}(\Omega)$ defined by

$$
\begin{aligned}
& \alpha_{-}(A)=\sup \{\alpha(B) \mid B \in \mathcal{A}(\Omega), B \Subset A\} \\
& \alpha_{+}(A)=\inf \{\alpha(B) \mid B \in \mathcal{A}(\Omega), A \Subset B\}
\end{aligned}
$$

for every $A \in \mathcal{A}(\Omega)$, with the usual convention $\inf \emptyset=+\infty$. It is easy to prove (see, for instance, proposition 4.7 in [16]) that the set

$$
\mathcal{R}(\alpha)=\left\{A \in \mathcal{A}(\Omega) \mid a_{-}(A)=\alpha_{+}(A)\right\}
$$

is rich in $\mathcal{A}(\Omega)$. Moreover the following result holds: 
Lemma 5.13. - Let $\alpha: \mathcal{A}(\Omega) \rightarrow \mathbb{R}$ be an increasing function and let $\mathcal{F}(\alpha)$ be the set of all $B \in \mathcal{A}(\Omega)$ for which

$$
\{A \in \mathcal{A}(\Omega) \mid A \cap B \in \mathcal{R}(\alpha)\}
$$

is rich in $\mathcal{A}(\Omega)$. Then $\mathcal{F}(\alpha)$ is rich in $\mathcal{A}(\Omega)$.

Proof. - Fix a chain $\left(B_{s}\right)_{s \in \mathbb{R}}$ in $\mathcal{A}(\Omega)$ and consider a countable set $\mathcal{D}=\left\{U_{k} \mid k \in \mathbb{N}\right\} \subseteq \mathcal{A}(\Omega)$ which is dense in $\mathcal{A}(\Omega)$. For every $k \in \mathbb{N}$ it is possible to find a chain $\left(A_{s}^{k}\right)_{s \in \mathbb{R}}$ such that $A_{0}^{k}=U_{k}$ and the set $\left\{A_{s}^{k} \mid s<0\right\}$ is cofinal in $U_{k}$. For every $k \in \mathbb{N}$ and for every rational $q,\left(B_{\boldsymbol{s}} \cap A_{\boldsymbol{s}+q}^{k}\right)_{\boldsymbol{s} \in \mathbb{R}}$ is a chain in $\mathcal{A}(\Omega)$; since $\mathcal{R}(\alpha)$ is rich in $\mathcal{A}(\Omega)$, there exists a set $T_{k, q} \subseteq \mathbb{R}$, at most countable, such that $B_{s} \cap A_{s+q}^{k} \in \mathcal{R}(\alpha)$ for every $s \in \mathbb{R} \backslash T_{k, q}$. Define $T=\bigcup\left\{T_{k, q} \mid k \in \mathbb{N}, q \in \mathbb{Q}\right\} ; T$ is at most countable and

$$
B_{\boldsymbol{s}} \cap A_{\boldsymbol{s}+q}^{k} \in \mathcal{R}(\alpha) \text { for every } s \in \mathbb{R} \backslash T, \quad k \in \mathbb{N} \text {, and } q \in \mathbb{Q} \text {. }
$$

Let us fix $s \in \mathbb{R} \backslash T$ and put $\mathcal{D}_{s}=\left\{A_{s+q}^{k} \mid k \in \mathbb{N}, q \in \mathbb{Q}\right\}$; we now show that $\mathcal{D}_{s}$ is dense in $\mathcal{A}(\Omega)$. Consider $G_{1}$ and $G_{2}$ in $\mathcal{A}(\Omega)$ with $G_{1} \Subset G_{2}$ and let $U_{k} \in \mathcal{D}$ be such that $G_{1} \Subset U_{k} \Subset G_{2}$. Since $\left\{A_{t}^{k} \mid t<0\right\}$ is cofinal in $U_{k}$, there exists $\sigma<0$ with $G_{1} \Subset A_{\sigma}^{k} \Subset U_{k}$. Let now $q \in \mathbb{Q}$ be such that $\sigma<s+q<0$. Then

$$
A_{s+q}^{k} \in \mathcal{D}_{s} \quad \text { and } \quad G_{1} \Subset A_{\sigma}^{k} \Subset A_{s+q}^{k} \Subset U_{k} \Subset G_{2} .
$$

We conclude that $\mathcal{D}_{s}$ is dense in $\mathcal{A}(\Omega)$.

Keep $s$ fixed in $\mathbb{R} \backslash T$; by (5.30) we have $B_{s} \cap A \in \mathcal{R}(\alpha)$ for every $A \in \mathcal{D}_{\boldsymbol{s}}$, i.e.

$$
\alpha_{-}\left(B_{s} \cap A\right)=\alpha_{+}\left(B_{s} \cap A\right)
$$

for every $A \in \mathcal{D}_{s}$. Since the increasing functions $A \mapsto \alpha_{-}\left(B_{s} \cap A\right)$ and $A \mapsto \alpha_{+}\left(B_{s} \cap A\right)$ coincide on a dense set, equality (5.31) actually holds for a rich family of sets $A$ (see proposition 4.8 in [16]). Therefore $B_{s} \in \mathcal{F}(\alpha)$ for every $s \in \mathbb{R} \backslash T$. We conclude that $\mathcal{F}(\alpha)$ is rich in $\mathcal{A}(\Omega)$.

Proof of theorem 5.12. - Assume that

$$
K_{0}\left(\psi_{h}\right) \rightarrow K_{0}(\psi) \quad \text { in } H_{0}^{m}(\Omega)
$$

and that $K_{0}(\psi) \neq \emptyset$. Then (b) follows immediately from definition 1.7 of Mosco convergence. Let us prove (a). By theorem 4.1 there exist a dense set $D$ in $\mathbb{R}$ and a set $\mathcal{F} \subseteq \mathcal{A}(\Omega)$, cofinal in $\Omega$, such that

$$
K_{0}\left(\mathbf{1}_{\left\{\psi_{h}>t\right\} \cap B}\right) \rightarrow K_{0}\left(\mathbf{1}_{\{\psi>t\} \cap B}\right) \text { in } H_{0}^{m}(\Omega)
$$


for every $t \in D$ and every $B \in \mathcal{F}$. As observed in theorem 4.1, we may suppose that $\mathcal{F}$ consists of elements $B \Subset \Omega$. Then, by applying theorem 5.1, we get that for every $t \in D$ and $B \in \mathcal{F}$ there exists a set $\mathcal{R}(t, B) \subseteq \mathcal{A}(\Omega)$, rich in $\mathcal{A}(\Omega)$, such that

$$
\lim _{h \rightarrow+\infty} C_{m, 2}\left(\left(\left\{\psi_{h}>t\right\} \cap B\right) \cap A\right)=C_{m, 2}((\{\psi>t\} \cap B) \cap A)
$$

for every $A \in \mathcal{R}(t, B)$. Since we may suppose $D$ countable, for every $B \in \mathcal{F}$ the set $\mathcal{R}(B)=\bigcap\{\mathcal{R}(t, B) \mid t \in D\}$ is rich in $\mathcal{A}(\Omega)$ and (5.32) holds for every $t \in D, B \in \mathcal{F}$ and $A \in \mathcal{R}(B)$. It is now easy to see that the set

$$
\mathcal{R}=\bigcup_{B \in \mathcal{F}}(\mathcal{R}(B) \cap \mathcal{A}(B))
$$

is rich in $\mathcal{A}(\Omega)$ and satisfies condition (a).

Assume now conditions (a) and (b). Let $D$ and $\mathcal{R}$ be as in (a). For every $t \in D$ define $\alpha_{t}(A)=C_{m, 2}(\{\psi>t\} \cap A)$ whenever $A \in \mathcal{A}(\Omega)$. From condition (a) it easily follows that if $A$ is in the set $\mathcal{R}\left(\alpha_{t}\right)$ introduced in (5.29), then $A$ satisfies (5.28) (indeed, if $\alpha_{t}^{\prime}(A)=\liminf C_{m, 2}\left(\left\{\psi_{h}>t\right\} \cap A\right)$ and $\alpha_{t}^{\prime \prime}(A)=\limsup C_{m, 2}\left(\left\{\psi_{h}>t\right\} \cap A\right)$, then (a) implies $\left(\alpha_{t}\right)_{-}=\left(\alpha_{t}^{\prime}\right)_{-}=$ $\left(\alpha_{t}^{\prime \prime}\right)_{-}$and $\left.\left(\alpha_{t}\right)_{+}=\left(\alpha_{t}^{\prime}\right)_{+}=\left(\alpha_{t}^{\prime \prime}\right)_{+}\right)$. By lemma 5.13, $\mathcal{F}\left(\alpha_{t}\right)$ is rich in $\mathcal{A}(\Omega)$ and, since it is not restrictive to assume $D$ countable, also the set $\mathcal{F}=\bigcap_{t \in D} \mathcal{F}\left(\alpha_{t}\right)$ is rich in $\mathcal{A}(\Omega)$.

Therefore, for every $t \in D$ and $B \in \mathcal{F}$ there exists a set $\mathcal{R}(t, B) \subseteq \mathcal{A}(\Omega)$, rich in $\mathcal{A}(\Omega)$, such that $A \cap B \in \mathcal{R}\left(\alpha_{t}\right)$ for every $A \in \mathcal{R}(t, B)$, hence

$$
\lim _{h \rightarrow+\infty} C_{m, 2}\left(\left(\left\{\psi_{h}>t\right\} \cap B\right) \cap A\right)=C_{m, 2}((\{\psi>t\} \cap B) \cap A)
$$

for every $A \in \mathcal{R}(t, B)$. We may assume that if $B \in \mathcal{F}$ then $B \Subset \Omega$; hence theorem 5.1 yields

$$
K_{0}\left(\mathbf{1}_{\left\{\psi_{h}>t\right\} \cap B}\right) \rightarrow K_{0}\left(\mathbf{1}_{\{\psi>t\} \cap B}\right) \text { in } H_{0}^{m}(\Omega)
$$

for every $t \in D$ and every $B \in \mathcal{F}$. We are now in a position to apply theorem 4.1 and conclude that

$$
K_{0}\left(\psi_{h}\right) \rightarrow K_{0}(\psi) \quad \text { in } H_{0}^{m}(\Omega)
$$

and that $K_{0}(\psi) \neq \emptyset$. 
Finally, we state a simple consequence of theorem 5.12 which can be proved as theorem 4.4 and in which the conditions for the Mosco convergence are expressed only trhough the capacities of the level sets of the obstacles.

ThEOREM 5.14. - Let $\left(\psi_{h}\right)$ be a sequence of functions from $\Omega$ into $\overline{\mathbb{R}}$ and let $\psi$ be a function from $\Omega$ into $\mathbb{R}$. Assume, in addition, the existence of a set $\Omega^{\prime} \Subset \Omega$ such that $\psi, \psi_{h} \leq 0(m, p)$-q.e. on $\Omega \backslash \Omega^{\prime}$ for every $h \in \mathbb{N}$. Then

$$
K_{0}\left(\psi_{h}\right) \rightarrow K_{0}(\psi) \quad \text { in } \quad H_{0}^{m}(\Omega) \quad \text { and } \quad K_{0}(\psi) \neq \emptyset
$$

if and only if the following conditions $\left(a^{\prime}\right)$ and $\left(b^{\prime}\right)$ are satisfied:

$\left(a^{\prime}\right)$ there exist a dense set $D$ in $\mathbb{R}$ and a set $\mathcal{R} \subseteq \mathcal{A}(\Omega)$, rich in $\mathcal{A}(\Omega)$, such that

$$
\lim _{h \rightarrow+\infty} C_{m, 2}\left(\left\{\psi_{h}>t\right\} \cap A\right)=C_{m, 2}(\{\psi>t\} \cap A)
$$

for every $t \in D$ and $A \in \mathcal{R}$;

(b) $\quad \lim _{t \rightarrow+\infty} \limsup _{h \rightarrow+\infty} \int_{t}^{+\infty} C_{m, 2}\left(\left\{\psi_{h}>s\right\}\right)(s-t)^{p-1} \mathrm{~d} s=0$.

\section{Acknowledgments}

I would like to express my sincere gratitude to Professor Gianni Dal Maso for the attention and the scientific learning he placed at my disposal while following this research.

\section{References}

[1] AdAms (D.R) - - Sets and functions of finite $L^{p}$-capacity, Indiana Univ. Math. J. 27 (1978) pp. 283-291.

[2] ADAMS (D.R) - - $L^{p}$-capacitary integrals with some applications, Proc. Symposia in Pure Math., vol. XXXV, Part I (1979) pp. 359-367.

[3] Atrouch (H.) . - Variational convergence for functions and operators, Pitman, London (1984).

[4] Attouch (H.) and Picard (C.) .- Problèmes variationnels et théorie du potentiel non linéaire, Ann. Fac. Sci. Toulouse Math. (5) 1 (1979) pp. 89-136.

[5] Attouch (H.) and Picard (C.) . - Inéquations variationnelles avec obstacles et espaces fonctionnels en théorie du potentiel, Applicable Analysis 12 (1981) pp. 287-306. 
[6] Boccardo (L.) and Murat (F.).- Nouveaux résultats de convergence dans des problèmes unilatéraux,

Nonlinear partial differential equations and their applications, Collège de France seminar, vol. II, pp. 64-85, Res. Notes in Math., Pitman, London (1982).

[7] Choquet (G.) . - Lectures on analysis, vol. I, Mathematics Lecture Note Series, Benjamin, Inc. (1969).

[8] DaL Maso (G.) .- On the integral representation of certain local functionals, Ricerche Mat. 32 (1983) pp. 85-113.

[9] Dal Maso (G.) - - Some necessary and sufficient conditions for the convergence of sequences of unilateral convex sets, J. Funct. Anal. 62 (1985) pp. 119-159.

[10] Dal Maso (G.) .- And Introduction to $\Gamma$-convergence, Corso S.I.S.S.A. 1986/87, Trieste.

[11] Dal Maso (G.) . - $\Gamma$-convergence and $\mu$-capacities, Ann. Scuola Norm. Sup. Pisa Cl. Sci. (4) 14 (1987) pp. 423-464.

[12] Dal Maso (G.) and Defranceschi (A.) - - Limits of nonlinear Dirichlet problems in varying domains, Manuscripta Math. 61 (1988) pp. 251-278.

[13] Dal Maso (G.) and Modica (L.) . - A general theory of variational functionals, Topics in functional analysis (1980-1981) pp. 149-221, Quaderni Scuola Norm. Sup. Pisa, (1981).

[14] Dal Maso (G.) and PAderni (G.) - - Variational inequalities for the biharmonic operator with variable obstacles, Ann. Mat. Pura Appl. (4) 153 (1988) pp. 203-227.

[15] De Giorgi (E.) and Franzoni (T.) - - Su un tipo di convergenza variazionale, Rend. Sem. Mat. Brescia 3 (1979) pp. 63-101.

[16] De Giorgi (E.) and Letta (G.) . - Une notion générale de convergence faible pour des fonctions croissantes d'ensemble,

Ann. Scuola Norm. Sup. Pisa Cl. Sci. (4) 4 (1977) pp. 61-99.

[17] FUGLEDE (B.). - The quasi topology associated with a countably subadditive set function,

Ann. Inst. Fourier (Grenoble) 21 (1971) pp. 123-169.

[18] Hedberg (L.I.) .- Spectral synthesis in Sobolev spaces and uniqueness of the Dirichlet problem, Acta Math. 147 (1981) pp. 237-264.

[19] Hedberg (L.I.) and WolfF (T.H.) . - Thin sets in nonlinear potential theory, Ann. Inst. Fourier (Grenoble) 33 (1983) pp. 161-188.

[20] Maz'Ya (V.G.) and Khavin (V.P.) . - Nonlinear potential theory, Russian Math. Surveys 27 (1972) pp. 71-148.

[21] Meyers (N.G.) - - A theory of capacities for potentials of functions in Lebesgue classes,

Math. Scand. 26 (1970) pp. 255-292.

[22] Mosco (U.) .- Convergence of convex sets and of solutions of variational inequalities,

Adv. in Math. 3 (1969) pp. 510-585. 
[23] Mosco (U.) . - An introduction to the approximate solution of variational inequalities,

Constructive aspects of functional analysis, Corso C.I.M.E. (1971), vol. II, pp. 499-685, Edizioni Cremonese, Roma (1973).

[24] PICARD (C.) .- Problème biharmonique avec obstacles variables,

Thèse, Univ. Paris-Sud (1984)

[25] Sonntag (Y.) . - Convergence au sens de U. Mosco,

Thèse, Univ. de Provence, Marseille (1980).

[26] STEIN (E.M.) . - Singular integrals and differentiability properties of functions, Princeton University Press, Princeton, New Jersey (1970). 